\title{
Directory of State and Local Government Laboratory Accreditation/Designation Programs
}

Charles W. Hyer, Editor

Standards Services Division

Technology Services

National Institute of Standards and Technology

Gaithersburg, MD 20899-2100

(Supersedes NIST Spec. Publ. 815: 1991 Edition)

January 2003 


\section{FOREWARD}

This directory is a guide to laboratory accreditation and similar types of programs conducted by state and local government agencies. These programs accredit or designate laboratories or other entities to conduct testing to assist the agencies in carrying out their responsibilities. Such accreditation or designation is based on some type of assessment regarding the capability of the laboratory to conduct the testing. However, the nature of such assessments varies considerably from agency to agency.

Laboratory accreditation and related efforts provide some assurance regarding the technical proficiency and competence of an entity to assess a product's or service's conformance to a set of prescribed standards. Many sectors of the economy may be interested in laboratory accreditation and related programs for a variety of economic, procurement, safety, or other considerations. Mutual acceptance of laboratory test results between states, local jurisdictions, and at the national and international levels can also serve as a basis for increased opportunities for trade.

This directory is an update of information contained in NBS GCR 84-472, Principal Aspects of U.S. Laboratory Accreditation Systems. This directory identifies state and local government laboratory accreditation/designation programs and notes the appropriate contact points within each agency and should be a valuable reference for all who operate, use, or rely on laboratory services. Entries in this directory are based primarily on information provided by each agency and reflect the agency's view of its activities. Interested parties may wish to review NISTIR 4576, Laboratory Accreditation in the United States, an introduction to the topic of laboratory accreditation, and NBS SP 808, Directory of Federal Government Laboratory

Accreditation/Designation Programs, a summary of programs conducted by the federal government. 


\section{ACKNOWLEDGMENTS}

This directory is based on my earlier publications in the field with the format expanded to be consistent with "NIST Special Publication 808, Directory of Federal Government Laboratory Accreditation/Designation Programs," edited by Maureen Breitenberg, Standards Services Division, NIST. She has provided important help and cooperation while monitoring this effort for NIST.

Special thanks are due to Ruth Schreiber of Schreiber's Quality Word Processing Services for her help in compiling this directory and managing all aspects of information processing. I would also like to thank the program officials who provided the information contained in this directory, without which this publication would not have been possible.

Charles W. Hyer

Editor 


\section{ABSTRACT}

This directory is a guide to laboratory accreditation and similar types of programs conducted by state and local government agencies. These programs accredit or designate laboratories or other entities to conduct testing to assist the agencies in carrying out their responsibilities. Such accreditation or designation is based on some type of assessment regarding the capability of the laboratory to conduct the testing. However, the nature of such assessments varies considerably from agency to agency.

Entries in this directory are based primarily on information provided by each state and local government agency and reflect the agency's view of its activities. Interested parties may wish to review NIST SP 808, Directory of Federal Government Laboratory Accreditation/Designation Programs, which contains information on similar programs conducted at the federal level.

The publication of this directory is part of ongoing NIST efforts to establish and maintain comprehensive information on standards, regulations, laboratory accreditation and certification programs and related information. This material answers the needs of government, industry, and the public for information on state and local government laboratory accreditation and related programs.

Key Words: accreditation; certification; designation; laboratory accreditation; laboratory designation; listing; proficiency testing; qualified laboratories; quality systems; standards; state government; testing 


\section{CONTENTS}

FORWARD -

ACKNOWLEDGMENTS

ABSTRACT

INTRODUCTION

PROGRAMS BY STATE/LOCAL/MUNICIPAL GOVERNMENTS

APPENDIX I - INDICES - 63

INDEX OF STATE/LOCAL/MUNICIPAL ACCREDITATION/DESIGNATION SYSTEMS -...-..- 65

INDEX BY FIELD OF TESTING-

INDEX BY PRODUCTS TESTED

APPENDIX II -- FORMAT USED FOR EACH ENTRY - 71

APPENDIX III -- INFORMATION AND PUBLICATIONS AVAILABLE FROM NIST

APPENDIX IV - OTHER LISTS OF STATE LABORATORY ACCREDITATION/DESIGNATION BODIES

OR RELATED ORGANIZATIONS - 85

LIST OF STATE NELAP RECOGNIZED ACCREDITING AUTHORITIES

LIST OF CLIA STATE SURVEY AGENCIES

LIST OF STATE GRADE 'A' MILK SANITATION PERSONNEL ................................... 97

LIST OF STATE CERTIFICATION OFFICERS FOR DRINKING WATER LABORATORIES .............121

LIST OF STATE CONTACT INFORMATION AVAILABLE FROM FEDERAL

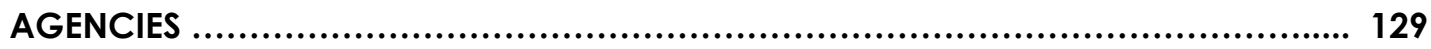

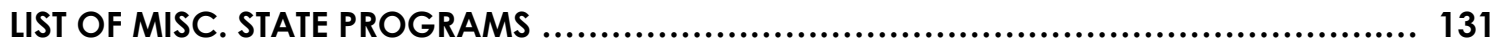




\section{INTRODUCTION}

\section{BACKGROUND}

Achieving national and international acceptance of the test results produced by competent testing laboratories has become a significant issue in discussions on reducing barriers to trade. Laboratory accreditation is recognized worldwide as an important means of assuring that laboratories are competent to conduct the required testing ${ }^{1}$. Widely accepted test results can greatly facilitate product ${ }^{2}$ entry into domestic and foreign markets. It is therefore important to understand the differences among laboratory accreditation programs and the impact that such differences can have on the confidence that can be placed in the technical competence of the laboratories they accredit.

The number of U.S. laboratory accreditation programs that evaluate the competence of testing and calibration laboratories and their compliance with specific and often differing technical requirements has created problems in achieving the free flow of goods and services within the United States. Not all U.S. laboratory accreditation programs are technically equivalent. This is especially true at the State and local government levels. Depending on how laboratories are assessed and what technical and other requirements are imposed, different laboratory accreditation programs can provide varying degrees of assurance in the technical competence of a laboratory to assess a product's conformance to a set of prescribed standards. ${ }^{3}$

It should be noted that the National Cooperation for Laboratory Accreditation [NACLA] has attempted to address this problem. NACLA was established as a nonprofit corporation to achieve a coordinated U.S. system for the accreditation of testing and calibration laboratories to eliminate unnecessary duplication and inefficiencies in both public and private sector use of laboratory accreditation. NACLA evaluates laboratory accreditation bodies $[\mathrm{ABs}]$ and grants recognition to those that meet the NACLA requirements. NACLA requirements include conformance to the international standard for laboratory accreditation competence, International Standards Organization/International Electrotechnical Commission [ISO/IEC] Guide 58: 1993, Calibration and testing laboratory accreditation systemsGeneral requirements for operation and recognition. ABs undergo a rigorous peer assessment and

\footnotetext{
$1_{15}$ CFR Part 287 Guidance on Federal Conformity Assessment Activities states that: "testing means the action of carrying out one or more technical operations (tests) that determine one or more characteristics or performance of a given product, material, equipment, organism, person's qualifications, physical phenomenon, process, or service according to a specified technical procedure (test method)." A laboratory is a body that conducts testing. International Organization for Standardization/International Electrotechnical Commission (ISO/IEC) Guide 58: 1993 defines laboratory as a "body that calibrates and/or tests."

2 For purposes of this directory, the term "product" includes: products, materials, equipment, organisms, person's qualifications, physical phenomena, processes, and/or services.

315 CFR Part 287 Guidance on Federal Conformity Assessment Activities states that accreditation "means a procedure used to provide formal notice that a body or person is competent to carry out specific tasks. These tasks include: sampling and testing; inspection; certification; and registration." It also notes that for some agencies, accreditation may mean that a body or person meets requirements defined in a specific section(s) of a regulation. "The referenced section(s) may include only limited requirements for demonstration of technical competency." International Organization for Standardization/International Electrotechnical Commission (ISO/IEC) Guide 58: 1993 defines laboratory accreditation as the: "Procedure by which an authoritative body gives formal recognition that a body or person is competent to carry out specific tests. "The term laboratory accreditation may cover the recognition of both the technical competence and the impartiality of a testing laboratory or only its technical competence.
} 
those ABs that pass are invited to sign the NACLA mutual recognition arrangement (MRA). Laboratories accredited by NACLA MRA signatories are required to conform to requirements contained in ISO/IEC 17025: 1999, General Requirements for the Competence of Calibration and Testing Laboratories and comply with requirements for proficiency testing. Under the MRA, signatories agree to recognize the technical competence of other MRA signatories. NACLA hopes that this MRA process will promote the recognition and worldwide acceptance of competent U.S. laboratory accreditations. NACLA is also involved at the international level as a stakeholder member of the International Laboratory Cooperation (ILAC), an international cooperation among various laboratory accreditation schemes operated throughout the world.

Laboratory accreditation can also have a major effect on the confidence that can be placed in the outcomes of product certification programs. While laboratory accreditation and product certification are two distinct areas, these two activities are closely related and are encompassed under the term "conformity assessment." The competence of laboratories which conduct the required testing within a certification or approval (e.g., building/safety codes) system is as vital in securing acceptance of the results of that certification/approval as is the adequacy of the standards on which the certification is based. All conformity assessment activities, ${ }^{4}$ including laboratory accreditation and/or product certification, that are used (or not used) in a conformity assessment program or system have a major impact on the confidence that can be placed in the outcome of that program or system. Assurance of laboratory competence is vital to the effectiveness and acceptance of many conformity assessment program and systems.

Laboratory accreditation can therefore be an important element in a conformity assessment process or system and in the national and international acceptance of conformity assessment results. As noted before, such acceptance can have a major impact on national and international trade. It is therefore useful for government agencies and industry to understand the nature and requirements of U.S. laboratory accreditation programs conducted at the state and local government levels and how these programs may be having a positive or negative impact on the national goals of reducing unnecessary duplication and complexity in the U.S. conformity assessment system and gaining national and international acceptance of U.S. conformity assessment results.

\section{PURPOSE OF THIS DIRECTORY}

The 1991 edition of SP 815 - Directory of State and Local Government Laboratory Accreditation/Designation Programs was an extremely popular reference. Thousands of copies have been distributed in the United States and abroad to those interested in State laboratory accreditation or designation requirements. This edition is an update of the 1991 edition and is designed to be a companion document to other directories and conformity assessment related information published by NIST. Its development is a response to numerous requests for current information on state and local government laboratory accreditation/designation programs in light of the increasing importance that laboratory accreditation/designation plays in the international trade arena. This directory is intended to

\footnotetext{
4 Conformity assessment activities include any activity concerned with determining directly or indirectly that requirements are fulfilled. Conformity assessment includes: sampling and testing; inspection; supplier's declaration of conformity; certification; and management system assessment and registration. It also includes the accreditation of laboratories, inspection bodies, certifiers and registrars, and the recognition (usually by a government agency) of the competence of an accreditation program.
} 
provide some insight into differences in requirements and "technical equivalence" of programs conducted at the State and local government level within the United States.

This updated directory is expected to be used by many to answer national and international inquiries about the existence and requirements of State and local government laboratory accreditation programs.

\section{CONTENT OF THIS DIRECTORY}

This directory provides information on State, local and municipal laboratory accreditation and similar type programs (systems) that accredit or designate (e.g., approve, accept, or list) laboratories or related entities to conduct testing activities to assist State, local and municipal agencies in carrying out their responsibilities. The entries include both regulatory and procurement programs that accredit/designate laboratories or related entities within the scope of those programs.

All accreditation or designation programs listed in this directory include some type of assessment regarding the capability of the laboratory/entity to conduct the specified inspection and testing activities. The type and degree of such assessments, however, vary greatly by the program. It should be noted that the entries in this directory are based primarily on previously published information, and information provided by the State, local or municipal agency, that reflect the agency's view of its activities.

Each entry contains a description of the program, information on the date that the program was initiated, the authority under which the program is conducted, the fields of testing being accredited/designated (based on categories defined by ASTM 1224-94 Standard Guide for Categorizing Fields of Testing for Laboratory Accreditation Purposes where appropriate), the products directly or indirectly affected by the testing, program requirements, availability of related publications, and other information.

\section{DIRECTORY FORMAT}

States, city and local government agency entries in this directory are organized by State. City and local government agency entries follow the State entries. Appendix I contains several indices, including a product index. A description of the format used for each entry is contained in Appendix II. Appendix III contains a list of other NIST publications of potential interest to the reader. Appendix IV contains lists of additional state laboratory accreditation/designation bodies or related organizations.

\section{PROGRAM DIFFERENCES}

Requirements for State and local government laboratory accreditation/designation programs vary substantially. State programs arising from primacy responsibilities assumed from the U.S.

Environmental Protection Agency (EPA) tend to include a review of documentation on: aspects of an organization's structure; personnel; equipment and facilities; equipment calibration and maintenance procedures; quality control program; record keeping procedures; availability and use of operational/quality manuals; content and quality of actual test reports; testing conditions, methods and 
procedures; and/or sample handling and selection procedures. They also tend to include an on-site inspection of the laboratory's equipment and facilities, and/or the use of a proficiency testing program. ${ }^{5}$

State, local and municipal programs designed to accredit/designate laboratories that test products and equipment for compliance to state and local government-adopted standards and codes tend to concentrate on initial documentation reviews and on-site assessments, but have few program follow-up or monitoring requirements. For example, some state and local government laboratory accreditation and designation programs have resulted from the adoption of national consensus standards and codes, such as the National Fire Protection Association's (NFPA) National Electrical Code (NEC). Certain NFPA standards and codes require product testing, safety evaluations, and approvals (or certifications) by a "nationally recognized listing and testing agency" for specified products to be acceptable for installation or use. Although the original NFPA standards did not define "nationally recognized listing and testing agency," various organizations were listed elsewhere in NFPA documents, including Underwriters Laboratories (UL) and Factory Mutual Research Corporation (FM). The development and implementation of programs to add additional laboratories to those traditionally named, as in NFPA documents, was a major motivation for the establishment of some state and local government accreditation/ designation programs. However, no surveillance requirement was included in the NFPA documents that recognized UL and FM, few of the programs intended to identify additional laboratories as having essentially the same capabilities as UL, FM or certain other "grandfathered" nationally recognized listing and testing agencies that include surveillance requirements within their programs.

It should be noted that the U.S. Department of Labor's Occupational Safety and Health Administration [OSHA] conducts a "nationally recognized testing laboratory" (NRTL) program that recognizes third party certifiers as NRTLs. Electrical products and equipment used in the workplace must be tested and listed or labeled by an NRTL. Such products are required to comply with a list of standards, submitted by the NRTL, which OSHA deems appropriate. NRTL recognition is valid for a five-year period, at which time recognition must be renewed. OSHA is required to audit each NRTL annually to verify that it continues to meet requirements for recognition. Many states that have adopted the NEC recognize NRTLs as their "nationally recognized listing and testing agencies." So while the state may not conduct surveillance activities directly, they rely on the OSHA program to ensure that NRTLs remain in compliance with the NRTL program's requirements.

The adoption of model building codes, such as the International Code Council's [ICC] International Building Code, that require that specified products conform to applicable code requirements has generated the need for additional programs to accredit/designate laboratories that can test specified products to these requirements. The first international, or I-Code, was the International Plumbing Code published in 1995. By 2000, a complete family of 11 construction codes was available. The ICC Performance Code for Buildings and Facilities, a consolidation of three model codes, was published in 2001. Laboratories generally submit a list of all other State, local or municipal accreditations or Code Body generated evaluation reports to a State or local government authority as part of the process to gain approval to conduct product evaluations within that jurisdiction. These accreditations and reports serve as influential recommendations. However, each State and local "authority having jurisdiction" (AHJ) has the final responsibility for its own accreditations/ designations.

\footnotetext{
${ }^{5}$ ISO/IEC Guide 2:1996 defines laboratory proficiency testing as the: "determination of laboratory testing performance by means
} of interlaboratory test comparisons." 
In general, the requirements of each program have been tailored to meet the agency's specific needs. The reader should not assume that comparable programs, even those conducted by cities with the same State, will include the same types or number of assessment procedures or will provide similar assurance regarding a laboratory's competency.

\section{LIMITATION ON THE NUMBER OF PROGRAMS LISTED}

The programs listed in this directory are a sampling of the State and local government programs that are assumed to be in operation as a result of a number of factors. As with the previous directory, there is no definitive list of State and local government programs. Programs are operated by agencies within State and local governments, making it difficult to identify the appropriate agency or official. A number of State and local government officials declined to provide information on their programs. In some cases, appropriate officials were under time constraints and the provision of information was not deemed to be a high priority. In other cases, requests for information were declined because most laboratory accreditation/designation/approval programs are functions that support State or local government product acceptance programs required under applicable regulations. Accredited/designated laboratories are needed only to provide that State or local government authority with a way to identify products that comply with product regulations and requirements. Unless a manufacturer or supplier of a regulated product wishes to use a laboratory that is not already accredited/designated by the State or local authority, the authority has no need to accredit or designate a new laboratory. A number of laboratory accreditation/designation programs are therefore not generally operational. In addition, a number of authorities indicated that they do not wish to be identified as accrediting or even designating laboratories in a national/international publication because they are not funded to provide such services for any laboratory not currently accepted by the State or local government authority to provide regulated product information. Many State and local government officials have also expressed a concern with being listed as an accreditation/designation body because they do not want to be held responsible for a laboratory's activities outside the scope of their programs. Finally, another major reason for the decision to decline to provide program information for inclusion in this Directory was the changing criteria and documents being used within such programs and the number of state and local government authorities that are increasingly looking at a number of national laboratory accreditation body programs for possible use within their programs. In summary, while the programs listed in this directory provide a sampling of programs in use at the State and local government levels, the directory should not be construed as being a comprehensive list of all such programs.

The following chart summarizes the number and types of assessment criteria and procedures required by the 21 State and 11 local and municipal government laboratory accreditation/designation programs identified in this directory. The requirements were derived from those contained in ISO/IEC 17025, though they do not include all requirements contained in that standard.

\begin{tabular}{|l|l|l|l|}
\hline \multicolumn{2}{|l|}{ REQUREMENT } & Yes & No \\
\hline \hline 1. & Be a legal entity & 26 & 1 \\
\hline 2. & Be financially stable & 13 & 14 \\
\hline 3. & Be independent of mfrs./suppliers of products tested. & 14 & 13 \\
\hline 4. & Have an effective quality system & 26 & 1 \\
\hline 5. & Have procedures to prevent conflicts-of-interest & 20 & 7 \\
\hline
\end{tabular}




\begin{tabular}{|l|l|l|l|}
\hline 6. & Have a document control system & 19 & 8 \\
\hline 7. & Have a contract review process & 10 & 17 \\
\hline 8. & Have procedures for sub-contracting tests and calibrations & 16 & 11 \\
\hline 9. & Have a documented procurement process & 9 & 18 \\
\hline 10. & Have a complaints/appeals process & 11 & 16 \\
\hline 11. & Have a system to control nonconforming testing and/or calibrations & 21 & 6 \\
\hline 12. & Have a corrective/preventive action process & 23 & 4 \\
\hline 13. & Have an effective recordkeeping process & 25 & 2 \\
\hline 14. & Have documented record retention requirements & 20 & 7 \\
\hline 15. & Conduct internal audits of its quality system & 22 & 5 \\
\hline 16. & Have laboratory management review results of internal audits & 20 & 7 \\
\hline 17. & Have qualified personnel & 27 & 0 \\
\hline 18. & Have laboratory measurements traceable to national standards & 24 & 3 \\
\hline 19. & Use effective sampling techniques & 19 & 8 \\
\hline 20. & Have a process for handling/transport of test/calibration items & 13 & 14 \\
\hline 21. & Participate in a proficiency testing program & 20 & 7 \\
\hline 22. & Have adequate instrumentation facilities and equipment & 21 & 6 \\
\hline 23. & Ensure adequate equipment maintenance/calibration & 21 & 6 \\
\hline 24. & Attend program laboratory workshops/conferences & 7 & 20 \\
\hline 25. & Maintain other or related accreditations/approvals & 6 & 21 \\
\hline
\end{tabular}

Most State, local, and municipal government programs have requirements for independence (no conflictof-interest), equipment, facility, and personnel. However, while two-thirds of the State programs have on-site visit requirements, only one of the eleven local and municipal government agencies reported having such a requirement. A few State programs conduct periodic random or scheduled visits or require participation in proficiency testing, but no local or municipal program reported having such a surveillance procedure/requirement.

Some programs accredit or designate laboratories in specific areas (such as testing drinking water or milk) or for specific test methods, while others designate laboratories over several fields of testing, each of which encompasses a broad range of test methods.

\section{ACCEPTANCE OF ACCREDITATION PROGRAMS BY OTHER STATE, LOCAL AND MUNICIPAL GOVERNMENT AGENCIES}

Accreditation/designation by some State laboratory accreditation programs can be useful in helping a laboratory gain accreditation/designation by other State, local and municipal governments. This principle is also true for laboratories that have achieved accreditation under the National Institute of Standards and Technology's (NIST) National Voluntary Laboratory Accreditation Program (NVLAP) ${ }^{6}$ and/or acceptance by laboratory evaluation programs of model code bodies. Such accreditations or acceptances can also be useful in gaining accreditation/ designation by a State or local government authority. However, it should be noted that other than the programs named in legislation (e.g.,

${ }^{6}$ Additional information on NVLAP is available on the NVLAP website at: http://s.nist.gov/nvlap. 
Connecticut's requirement for NVLAP participation by construction materials testing laboratories), each State, local or municipal agency accredits or designates its own list of acceptable laboratories.

\title{
DIRECTORY CHANGES
}

All agencies listed in the 1991 edition of NIST SP 815, as well as over 150 new state and local government agencies identified through various sources, were contacted for information. For example, the International Association of Electrical Inspectors' [IAEI] compendium Summary of Electrical \& Building Code Requirements, Licensing Provisions and Laboratory Recognition at the State and Local Levels was consulted and appropriate organizations contacted. However, the response rate for organizations not listed in the 1991 edition of SP 815 was very low even though each organization was contacted several times for information.

State, local and municipal agencies are encouraged to notify NIST of any changes in their laboratory accreditation programs or of new programs. Such information should be sent to:

\author{
STANDARDS SERVICES DiVISION (SSD) \\ National Institute of Standards and Technology \\ 100 Bureau Drive, MS 2100 \\ Gaithersburg, MD 20899-2100 \\ e-mail: Maureen.Breitenberg@nist.gov \\ Phone: (301) 975-4031 \\ Fax: (301) 963-2871
}

\section{OTHER REFERENCES}

Parties interested in this area may also wish to review NIST Special Publication SP 903, 1996 Edition of Directory of U.S. Private Sector Product Certification Programs available on the internet at http://ts.nist.gov/cainfo. SP 903 contains information on a number of closely related programs, many of which are conducted primarily for recognition and use by State, local and municipal regulatory and procurement agencies. In addition, the reader may wish to review other NIST standards and conformity assessment publications listed in Appendix III.

Those interested in obtaining more information on NACLA can contact:

NACLA Secretariat

National Institute of Standards and Technology (NIST)

P.O. Box 4045

Gaithersburg, MD 20885-4045

Phone: (301) 975-6472

Fax: (301) 963-2871

URL Address: http://www.nacla.net 
Those interested in obtaining information on the International Laboratory Accreditation Cooperation (ILAC) can contact:

\section{ILAC Secretariat}

c/o NATA

7 Leeds Street,

Rhodes NSW 2138, Australia

Phone: +61 297368374

Fax: +61 297368373

Email: 1lac@,nata.asn.au

URL address: http://www.ilac.org/ 
DATE RECEIVED: 02/01

\begin{tabular}{|c|c|}
\hline DEPARTMENT/AGENCY : & ALABAMA DEPARTMENT OF ENVIRONMENTAL MANAGEMENT [ADEM] \\
\hline ADDRESS : & $\begin{array}{l}\text { ADEM Central Laboratory, } 1890 \text { A Cong. Dickson Drive, Montgomery, AL } \\
36109\end{array}$ \\
\hline & PHONE: $334-260-2770$ \\
\hline & E-MAIL: WRB@ADEM.state.al.us \\
\hline NAME OF PROGRAM: & Drinking Water Certification. \\
\hline PROGRAM DESCRIPTION: & $\begin{array}{l}\text { Program to certify (accredit) private sector laboratories to perform } \\
\text { drinking water chemical testing. }\end{array}$ \\
\hline DATE PROGRAM STARTED: & 1977 . \\
\hline FIELDS OF TESTING: & $\begin{array}{ll}\square \text { Acoustic and Vibration Testing } & \square \text { Mechanical Testing } \\
\square \text { Biological Testing } & \square \text { Metrology } \\
\bigotimes \text { Chemical Testing } & \square \text { Nondestructive Testing } \\
\square \text { Electrical Testing } & \square \text { Optics and Photometry } \\
\square \text { Ionizing Radiation } & \square \text { Thermal Testing } \\
\square \text { Other } & \end{array}$ \\
\hline $\begin{array}{l}\text { CATEGORIES OF PRODUCTS } \\
\text { COVERED BY THE PROGRAM: }\end{array}$ & Drinking water. \\
\hline $\begin{array}{l}\text { NUMBER OF LABS ACCREDITED/ } \\
\text { APPROVED : }\end{array}$ & 15 . \\
\hline $\begin{array}{l}\text { ACCREDITATION/APPROVAL } \\
\text { VALID FOR: }\end{array}$ & 1 year. \\
\hline NUMBER OF ASSESSORS: & 2. \\
\hline $\begin{array}{l}\text { MANDATORY ASSESSOR } \\
\text { QUALIFICATIONS : }\end{array}$ & 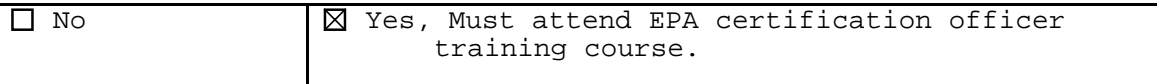 \\
\hline PARTICIPATION FEE: & $\begin{array}{l}\text { Yes, Cost information available from ADEM } \\
\text { Public Water Supply Branch, P.O. Box } \\
\text { 301463, Montgomery, AL } 36130 .\end{array}$ \\
\hline FEE SCHEDULE AVAILABLE: & $\begin{array}{l}\text { Yes, Fee schedule is available from ADEM Public } \\
\text { Water Supply Branch, P.O. Box 301463, } \\
\text { Montgomery, AL 36130. }\end{array}$ \\
\hline PROGRAM HAS AN APPEALS PROCEDURE : & $\begin{array}{c}\triangle \text { Yes, Appeals can be made to the Alabama } \\
\text { Department of Environmental Management. }\end{array}$ \\
\hline PROGRAM HAS A LOGO: & $\square$ Yes \\
\hline PROGRAM IS OPEN TO LABORATORIES: & $\begin{array}{ll}\text { 冈 All U.S. Private Sector } & \text { All U.S. Government } \\
\square \text { All Foreign Private Sector } & \square \text { All Foreign Government } \\
\square \text { Other } & \end{array}$ \\
\hline $\begin{array}{l}\text { AVAILABILITY OF PUBLISHED LIST OF } \\
\text { LABORATORIES: }\end{array}$ & 凶 Yes, Directories are available on request. \\
\hline $\begin{array}{l}\text { AVAILABILITY OF ASSESSMENT } \\
\text { CRITERIA/PROCEDURES: }\end{array}$ & $\begin{array}{l}\text { Procedures/requirements are available from the ADEM Division of } \\
\text { Public Water Supply, } 14000 \text { Coliseum Blvd., Montgomery, AL } 36130 .\end{array}$ \\
\hline
\end{tabular}




\begin{tabular}{|c|c|}
\hline $\begin{array}{l}\text { APPROVED/ACCREDITED LABORATORY } \\
\text { MANDATED REQUIREMENTS: }\end{array}$ & 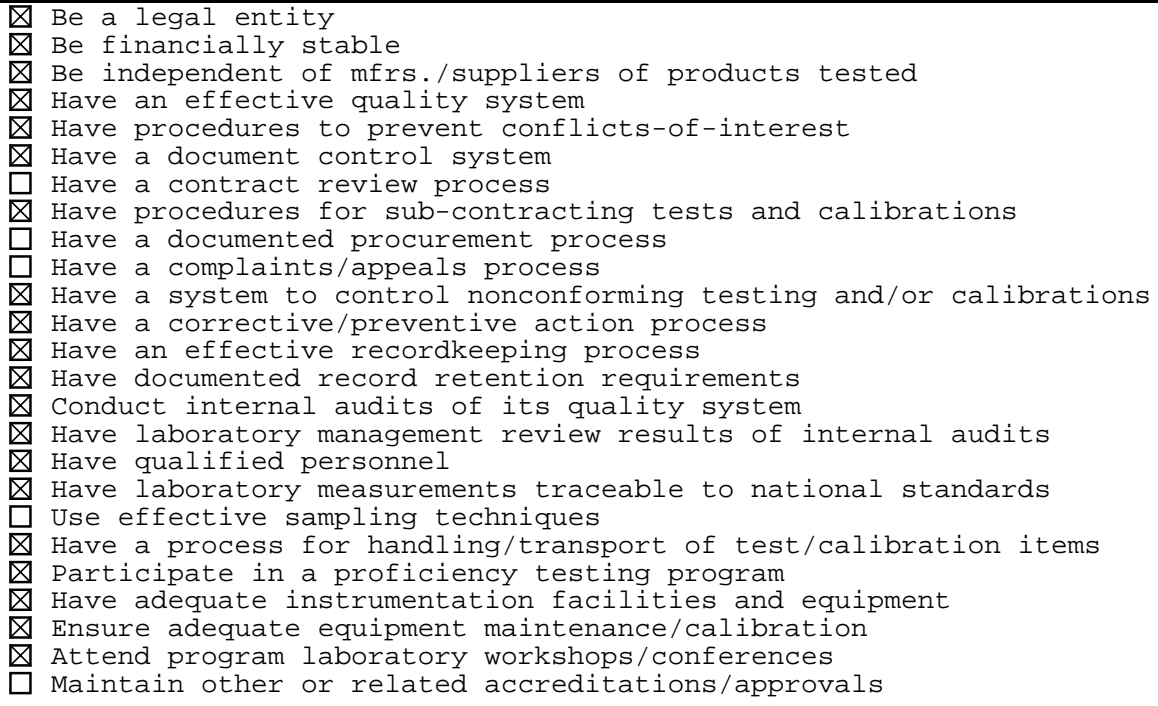 \\
\hline $\begin{array}{l}\text { STANDARD (S) WITH WHICH PROGRAM } \\
\text { CONFORMS : }\end{array}$ & 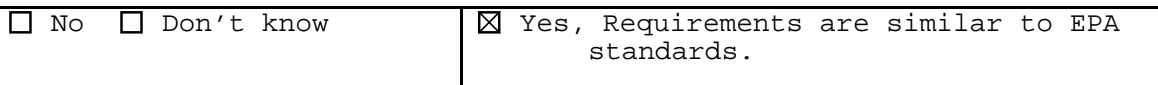 \\
\hline $\begin{array}{l}\text { PROGRAM RECOGNITION/MUTUAL } \\
\text { RECOGNITION ARRANGEMENTS: }\end{array}$ & None. \\
\hline
\end{tabular}


DATE RECEIVED:

\begin{tabular}{|c|c|}
\hline DEPARTMENT/AGENCY : & CALIFORNIA STATE DEPARTMENT OF HEALTH SERVICES \\
\hline \multirow[t]{3}{*}{ ADDRESS : } & ELAP Program Chief, 2151 Berkeley Way, Annex 2, Berkeley, CA 94704 \\
\hline & $\begin{array}{l}\text { INTERNET URL: } \\
\text { wWw.dhs.ca.gov/ps/ls/elapindex.htm }\end{array}$ \\
\hline & E-MAIL: gkulasin@dhs.ca.gov \\
\hline NAME OF PROGRAM: & Environmental Laboratory Accreditation Program (ELAP) \\
\hline PROGRAM DESCRIPTION: & $\begin{array}{l}\text { Accreditation/certification of environmental testing laboratories for } \\
\text { providing reliable analytical data for monitoring the quality of the } \\
\text { environment for the protection of public health in california. The } \\
\text { program covers certification for testing drinking water, wastewater, } \\
\text { hazaardous waste, pesticide residues in food and shellfish } \\
\text { sanitation. }\end{array}$ \\
\hline DATE PROGRAM STARTED: & January 1989. \\
\hline FIELDS OF TESTING: & $\begin{array}{ll}\text { Acoustic and Vibration Testing } & \square \text { Mechanical Testing } \\
\bigotimes \text { Biological Testing } & \square \text { Metrology } \\
\bigotimes \text { Chemical Testing } & \square \text { Nondestructive Testing } \\
\square \text { Electrical Testing } & \square \text { Optics and Photometry } \\
\text { Ionizing Radiation } & \square \text { Thermal Testing } \\
\text { Other } & \end{array}$ \\
\hline $\begin{array}{l}\text { CATEGORIES OF PRODUCTS } \\
\text { COVERED BY THE PROGRAM: }\end{array}$ & $\begin{array}{l}\text { Only certificates of NELAP accreditation and state ELAP certification } \\
\text { are issued. }\end{array}$ \\
\hline $\begin{array}{l}\text { NUMBER OF LABS ACCREDITED/ } \\
\text { APPROVED: }\end{array}$ & 800 \\
\hline $\begin{array}{l}\text { ACCREDITATION/APPROVAL } \\
\text { VALID FOR: }\end{array}$ & 2 years. \\
\hline NUMBER OF ASSESSORS : & 15 full time, 4 in training. \\
\hline $\begin{array}{l}\text { MANDATORY ASSESSOR } \\
\text { QUALIFICATIONS : }\end{array}$ & \begin{tabular}{|l|l|}
$\square$ No & Y Yes
\end{tabular} \\
\hline PARTICIPATION FEE : & $\triangle$ No \\
\hline FEE SCHEDULE AVAILABLE: & \begin{tabular}{|l|ll}
$\square$ No & Yes, $\$ 959.00+\$ 432.00$ for each field of testing.
\end{tabular} \\
\hline PROGRAM HAS AN APPEALS PROCEDURE: & \begin{tabular}{|l|l}
$\square$ No & $\bigotimes$ Yes, appeals made to Program Chief.
\end{tabular} \\
\hline PROGRAM HAS A LOGO: & $\begin{array}{l}\text { \ Yes, the National Environmental Laboratory } \\
\text { Accreditation Program Logo. }\end{array}$ \\
\hline $\begin{array}{l}\text { PROGRAM IS OPEN TO } \\
\text { LABORATORIES : }\end{array}$ & $\begin{array}{ll}\text { All U.S. Private Sector } & \text { All U.S. Government } \\
\text { All Foreign Private Sector } & \text { All Foreign Government } \\
\text { Other } & \end{array}$ \\
\hline $\begin{array}{l}\text { AVAILABILITY OF PUBLISHED LIST OF } \\
\text { LABORATORIES: }\end{array}$ & \begin{tabular}{|l|r|}
$\square$ No & $\begin{array}{r}\text { Yes, hard copies produced upon request. Also } \\
\text { available on the web at: } \\
\text { www.dhs.ca.gov/ps/ls/elapindex.htm }\end{array}$
\end{tabular} \\
\hline $\begin{array}{l}\text { AVAILABILITY OF ASSESSMENT } \\
\text { CRITERIA/PROCEDURES : }\end{array}$ & Available upon request. \\
\hline
\end{tabular}




\begin{tabular}{|c|c|}
\hline $\begin{array}{l}\text { APPROVED/ACCREDITED LABORATORY } \\
\text { MANDATED REQUIREMENTS: }\end{array}$ & $\begin{array}{l}\text { Be a legal entity } \\
\square \text { Be financially stable } \\
\square \text { Be independent of mfrs./suppliers of products tested } \\
\text { Have an effective quality system } \\
\text { Have procedures to prevent conflicts-of-interest } \\
\text { Have a document control system } \\
\square \text { Have a contract review process } \\
\square \text { Have procedures for sub-contracting tests and calibrations } \\
\square \text { Have a documented procurement process } \\
\square \text { Have a complaints/appeals process } \\
\text { Have a system to control nonconforming testing and/or calibrations } \\
\text { Have a corrective/preventive action process } \\
\text { Have an effective recordkeeping process } \\
\square \text { Have documented record retention requirements } \\
\square \text { Conduct internal audits of its quality system } \\
\square \text { Have laboratory management review results of internal audits } \\
\text { Have qualified personnel } \\
\text { Have laboratory measurements traceable to national standards } \\
\text { Hse effective sampling techniques } \\
\square \text { Have a process for handling/transport of test/calibration items } \\
\square \text { Participate in a proficiency testing program } \\
\bigotimes \text { Have adequate instrumentation facilities and equipment } \\
\text { Ensure adequate equipment maintenance/calibration } \\
\square \text { Attend program laboratory workshops/conferences } \\
\square \text { Maintain other or related accreditations/approvals }\end{array}$ \\
\hline $\begin{array}{l}\text { STANDARD (S) WITH WHICH PROGRAM } \\
\text { CONFORMS : }\end{array}$ & $\begin{array}{c}\text { Yes, conforms to National Environmental } \\
\text { Laboratory Accreditation (NELAC) } \\
\text { Program requirements. }\end{array}$ \\
\hline $\begin{array}{l}\text { PROGRAM RECOGNITION/MUTUAL } \\
\text { RECOGNITION ARRANGEMENTS : }\end{array}$ & $\begin{array}{l}\text { NELAC from NELAP program; USEPA from the Drinking Water Program; and } \\
\text { the States of Washington, Florida, Kansas, Nevada and Oregon. }\end{array}$ \\
\hline
\end{tabular}




\begin{tabular}{|c|c|}
\hline DEPARTMENT/AGENCY : & CALIFORNIA, CITY OF LOS ANGELES \\
\hline ADDRESS : & $\begin{array}{l}201 \text { N. Figueroa Street, Suit } 880 \\
\text { Los Angeles, CA } 90012\end{array}$ \\
\hline & INTERNET URL: srico@ladbs.lacity.org \\
\hline & FAX: $213-977-6272$ \\
\hline NAME OF PROGRAM: & Testing Agency Approval \\
\hline PROGRAM DESCRIPTION : & $\begin{array}{l}\text { Approval of all laboratories that test and list building, plumbing, } \\
\text { mechanical and electrical products whose published listings are } \\
\text { recognized by the Department after evidence of compliance to the } \\
\text { requirements of the Department. }\end{array}$ \\
\hline DATE PROGRAM STARTED: & Not applicable. \\
\hline FIELDS OF TESTING: & $\begin{array}{|ll|}\square \text { Acoustic and Vibration Testing } & \text { Mechanical Testing } \\
\square \text { Biological Testing } & \square \text { Metrology } \\
\square \text { Chemical Testing } & \square \text { Nondestructive Testing } \\
\square \text { Electrical Testing } & \square \text { Optics and Photometry } \\
\square \text { Ionizing Radiation } & \square \text { Thermal Testing } \\
\square \text { Other } & \end{array}$ \\
\hline $\begin{array}{l}\text { CATEGORIES OF PRODUCTS } \\
\text { COVERED BY THE PROGRAM: }\end{array}$ & $\begin{array}{l}\text { Building materials (structural steel, wood, concrete, roofing, glass, } \\
\text { insulations, etc.); mechanical (heating, ventilating); electrical (all } \\
\text { electrical equipment, and construction materials). }\end{array}$ \\
\hline $\begin{array}{l}\text { NUMBER OF LABS ACCREDITED/ } \\
\text { APPROVED : }\end{array}$ & 257. \\
\hline $\begin{array}{l}\text { ACCREDITATION/APPROVAL } \\
\text { VALID FOR: }\end{array}$ & 1 year. \\
\hline NUMBER OF ASSESSORS: & 6 . \\
\hline $\begin{array}{l}\text { MANDATORY ASSESSOR } \\
\text { QUALIFICATIONS : }\end{array}$ & \begin{tabular}{|l|l|}
$\square$ No & Yes. The goal is assessors that are either \\
& $\begin{array}{c}\text { experienced Senior Inspectors or senior } \\
\text { Engineers with testing laboratory } \\
\text { experience. }\end{array}$ \\
\end{tabular} \\
\hline PARTICIPATION FEE : & \Yes \\
\hline FEE SCHEDULE AVAILABLE: & $\begin{array}{c}\text { Q Yes. A copy of the fee schedule may be obtained } \\
\text { by calling the Department at 323-226-1638. }\end{array}$ \\
\hline PROGRAM HAS AN APPEALS PROCEDURE: & $\begin{array}{l}\text { 囚 Yes. The accreditation/approval decisions may be } \\
\text { appealed to the General Manager of the } \\
\text { Department and the Los Angeles Department } \\
\text { of Building and Safety Board of } \\
\text { Commissioners. }\end{array}$ \\
\hline PROGRAM HAS A LOGO: & $\square$ Yes \\
\hline $\begin{array}{l}\text { PROGRAM IS OPEN TO } \\
\text { LABORATORIES : }\end{array}$ & \begin{tabular}{|ll} 
All U.S. Private Sector & All U.S. Government \\
All Foreign Private Sector & All Foreign Government \\
$\square$ Other &
\end{tabular} \\
\hline $\begin{array}{l}\text { AVAILABILITY OF PUBLISHED LIST OF } \\
\text { DIRECTORIES AVAILABLE: }\end{array}$ & \begin{tabular}{l|l} 
Yes. A list is published in two parts under the \\
Department of Building and Safety Test \\
Lab/Training Publication: \\
Part "1 "Recognized Testing and Listing \\
Agencies" and \\
Part 2 "Testing Agencies."
\end{tabular} \\
\hline $\begin{array}{l}\text { AVAILABILITY OF ASSESSMENT } \\
\text { CRITERIA/PROCEDURES: }\end{array}$ & $\begin{array}{l}\text { Available in the Information Bulletin No. P/BC2001-59 (Formerly } \\
\text { Memorandum of General Distribution 93). }\end{array}$ \\
\hline
\end{tabular}




\begin{tabular}{|c|c|}
\hline $\begin{array}{l}\text { APPROVED/ACCREDITED LABORATORY } \\
\text { MANDATED REQUIREMENTS: }\end{array}$ & 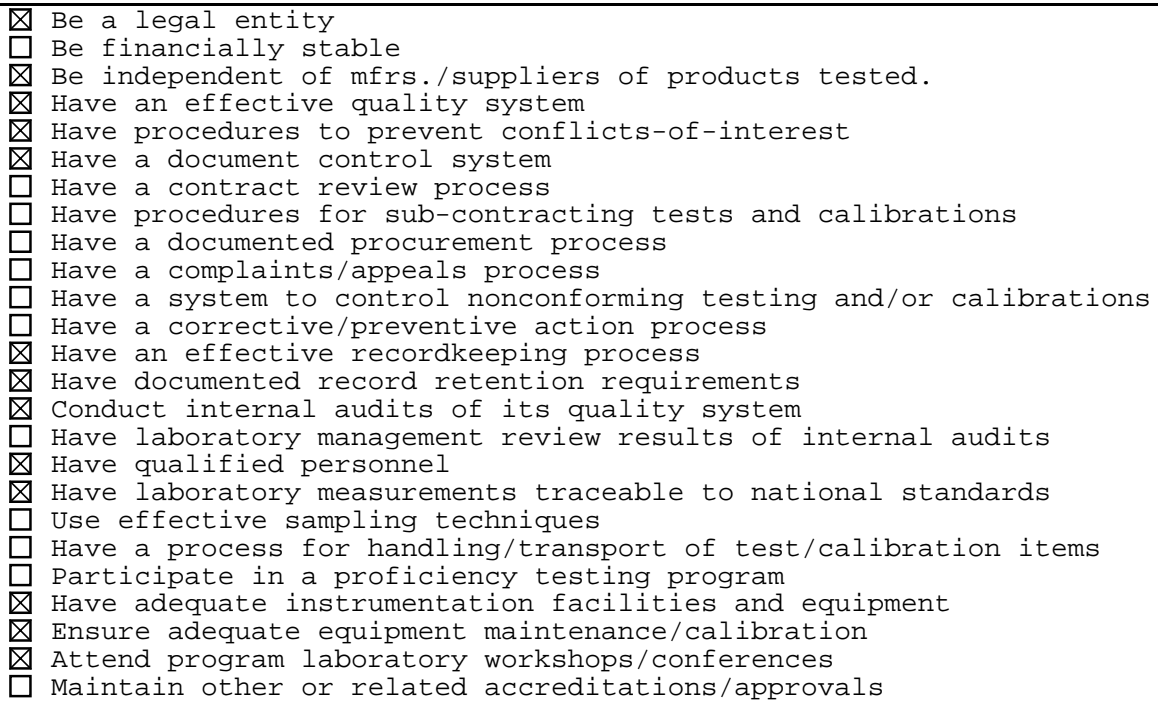 \\
\hline $\begin{array}{l}\text { STANDARD (S) WITH WHICH PROGRAM } \\
\text { CONFORMS: }\end{array}$ & $\begin{array}{c}\text { Yes. The Department's requirements for } \\
\text { laboratories are comparable to the } \\
\text { International Standard } \\
\text { Organization's (ISO). }\end{array}$ \\
\hline $\begin{array}{l}\text { PROGRAM RECOGNITION/MUTUAL } \\
\text { RECOGNITION ARRANGEMENTS: }\end{array}$ & None. \\
\hline
\end{tabular}




\begin{tabular}{|c|c|c|c|}
\hline DEPARTMENT/AGENCY : & \multicolumn{3}{|c|}{ FLORIDA DIVISION OF STATE FIRE MARSHAL } \\
\hline ADDRESS : & \multicolumn{3}{|c|}{200 E Gaines Street, Tallahassee, FL 32399-0400 } \\
\hline & \multicolumn{2}{|l|}{ PHONE : $850-413-3600$} & INTERNET URL: www.doi.state.fl.us \\
\hline & \multicolumn{2}{|l|}{ FAX: $850-922-1235$} & E-MAIL: $\quad$ clarkeddoi.state.fl.us \\
\hline NAME OF PROGRAM: & \multicolumn{3}{|c|}{ Fire Marshal's Forensic Laboratory } \\
\hline PROGRAM DESCRIPTION: & \multicolumn{3}{|c|}{$\begin{array}{l}\text { The Florida Fire Marshal operates a Forensic Laboratory for the } \\
\text { purpose of testing evidence from suspicious fires or explosions. On } \\
\text { occasion it must refer to and rely upon testing conducted by other } \\
\text { laboratories. In order to assure itself that these laboratories are } \\
\text { providing a quality service, the Forensic Laboratory may review the } \\
\text { contracted laboratory's methods, personnel, and services. }\end{array}$} \\
\hline DATE PROGRAM STARTED: & \multicolumn{3}{|l|}{1991} \\
\hline FIELDS OF TESTING: & \multicolumn{3}{|c|}{$\begin{array}{ll}\square \text { Acoustic and Vibration Testing } & \square \text { Mechanical Testing } \\
\square \text { Biological Testing } & \square \text { Metrology } \\
\text { Chemical Testing } & \square \text { Nondestructive Testing } \\
\text { Electrical Testing } & \square \text { Optics and Photometry } \\
\square \text { Ionizing Radiation } & \square \text { Thermal Testing } \\
\square \text { Other } & \end{array}$} \\
\hline $\begin{array}{l}\text { CATEGORIES OF PRODUCTS } \\
\text { COVERED BY THE PROGRAM: }\end{array}$ & \multicolumn{3}{|l|}{ Not applicable. } \\
\hline $\begin{array}{l}\text { NUMBER OF LABS ACCREDITED/ } \\
\text { APPROVED : }\end{array}$ & \multicolumn{3}{|l|}{ Not applicable. } \\
\hline $\begin{array}{l}\text { ACCREDITATION/APPROVAL } \\
\text { VALID FOR: }\end{array}$ & \multicolumn{3}{|l|}{ Not applicable. } \\
\hline NUMBER OF ASSESSORS: & \multicolumn{3}{|l|}{ Not applicable. } \\
\hline $\begin{array}{l}\text { MANDATORY ASSESSOR } \\
\text { QUALIFICATIONS : }\end{array}$ & \multicolumn{3}{|l|}{ 凶 No } \\
\hline PARTICIPATION FEE: & \multicolumn{3}{|l|}{ 凶No } \\
\hline FEE SCHEDULE AVAILABLE: & \multicolumn{3}{|l|}{ 凶 No } \\
\hline PROGRAM HAS AN APPEALS PROCEDURE : & \multicolumn{3}{|l|}{ 囚 No } \\
\hline PROGRAM HAS A LOGO: & 凶 No & \multicolumn{2}{|c|}{$\square$ Yes } \\
\hline $\begin{array}{l}\text { PROGRAM IS OPEN TO } \\
\text { LABORATORIES : }\end{array}$ & \multicolumn{3}{|c|}{$\begin{array}{ll}\text { All U.S. Private Sector } & \text { All U.S. Government } \\
\square \text { All Foreign Private Sector } & \square \text { All Foreign Government } \\
\square \text { Other } & \end{array}$} \\
\hline $\begin{array}{l}\text { AVAILABILITY OF PUBLISHED LIST OF } \\
\text { LABORATORIES: }\end{array}$ & \multicolumn{3}{|c|}{\begin{tabular}{|l|l}
$\bigotimes$ No & $\square$ Yes
\end{tabular}} \\
\hline $\begin{array}{l}\text { AVAILABILITY OF ASSESSMENT } \\
\text { CRITERIA/PROCEDURES : }\end{array}$ & \multicolumn{3}{|l|}{ Not applicable. } \\
\hline
\end{tabular}




\begin{tabular}{|c|c|}
\hline $\begin{array}{l}\text { APPROVED/ACCREDITED LABORATORY } \\
\text { MANDATED REQUIREMENTS: }\end{array}$ & 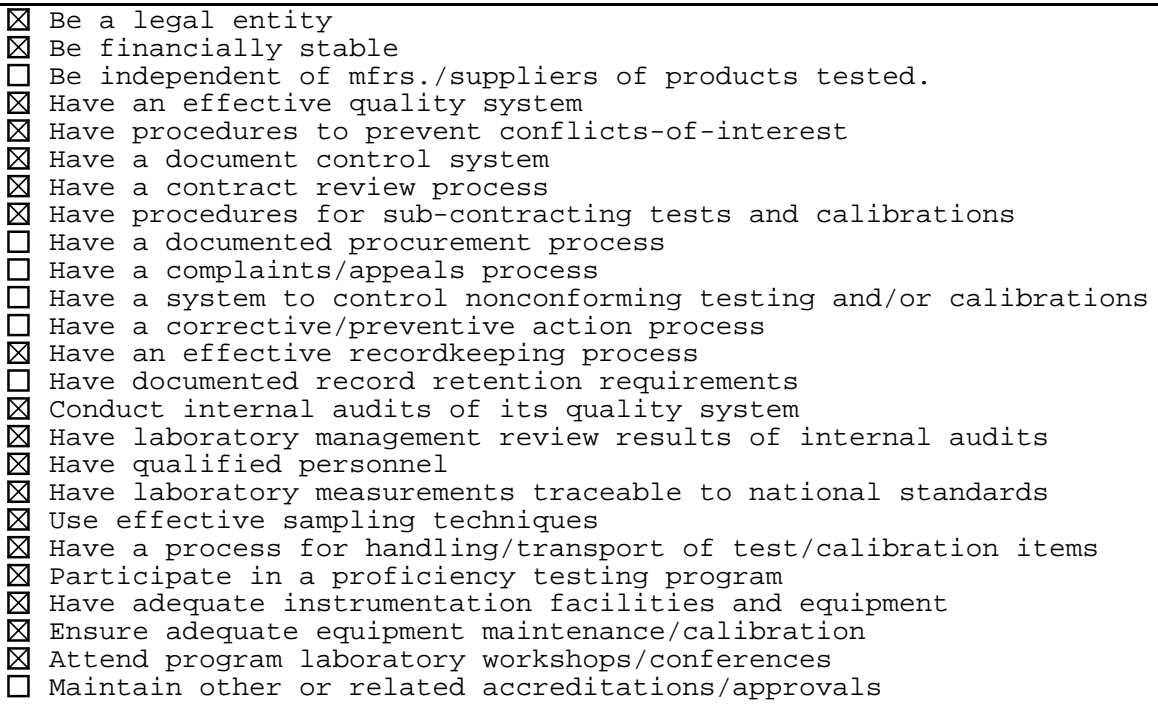 \\
\hline $\begin{array}{l}\text { STANDARD (S) WITH WHICH PROGRAM } \\
\text { CONFORMS: }\end{array}$ & $\square$ No $\otimes$ Don't know \\
\hline $\begin{array}{l}\text { PROGRAM RECOGNITION/MUTUAL } \\
\text { RECOGNITION ARRANGEMENTS : }\end{array}$ & Not applicable. \\
\hline
\end{tabular}




\begin{tabular}{|c|c|}
\hline DEPARTMENT/AGENCY : & FLORIDA-MIAMI-DADE PRODUCT CONTROL DIVISION \\
\hline ADDRESS : & $\begin{array}{l}\text { Metro-Dade Flagler Building, } 140 \text { West Flagler Street, Suite 1603, } \\
\text { Miami, FL 33130-1563 }\end{array}$ \\
\hline & INTERNET URL: wWw.BuildingCodeOnline.com \\
\hline & E-MAIL：RYR@CO.Miami-Dade.Fl.us \\
\hline NAME OF PROGRAM: & Miami-Dade Product Approval \\
\hline PROGRAM DESCRIPTION : & $\begin{array}{l}\text { Approval of building products used to protect the envelope of a } \\
\text { building from being breached. Products such as windows, doors, } \\
\text { hurricane shutters, roofing, prefabricated buildings, EIFs (Exterior } \\
\text { Insulation and Finish systems), wood truss plates, concrete plates, } \\
\text { steel fabrications, concrete blocks, impact resistant glass, store } \\
\text { fronts, and curtain walls. }\end{array}$ \\
\hline DATE PROGRAM STARTED: & 1957 \\
\hline FIELDS OF TESTING: & $\begin{array}{ll}\square \text { Acoustic and Vibration Testing } & \square \text { Mechanical Testing } \\
\square \text { Biological Testing } & \square \text { Metrology } \\
\square \text { Chemical Testing } & \square \text { Nondestructive Testing } \\
\square \text { Electrical Testing } & \square \text { Optics and Photometry } \\
\square \text { Ionizing Radiation } & \square \text { Thermal Testing } \\
\text { Other Hurricane Protection. } & \end{array}$ \\
\hline $\begin{array}{l}\text { CATEGORIES OF PRODUCTS } \\
\text { COVERED BY THE PROGRAM: }\end{array}$ & Construction. \\
\hline $\begin{array}{l}\text { NUMBER OF LABS ACCREDITED/ } \\
\text { APPROVED: }\end{array}$ & Available upon request. \\
\hline $\begin{array}{l}\text { ACCREDITATION/APPROVAL } \\
\text { VALID FOR: }\end{array}$ & 5 years. \\
\hline NUMBER OF ASSESSORS : & Available upon request. \\
\hline $\begin{array}{l}\text { MANDATORY ASSESSOR } \\
\text { QUALIFICATIONS : }\end{array}$ & $\triangle$ Yes \\
\hline PARTICIPATION FEE : & 凶 Yes, available at: www.buildingcodeonline.com. \\
\hline FEE SCHEDULE AVAILABLE: & 区 Yes, available at: www.buildingcodeonline.com. \\
\hline PROGRAM HAS AN APPEALS PROCEDURE: & $\begin{array}{c}\otimes \text { Yes, appeal may be made to: Building Code } \\
\text { Product Review Committee. }\end{array}$ \\
\hline PROGRAM HAS A LOGO: & 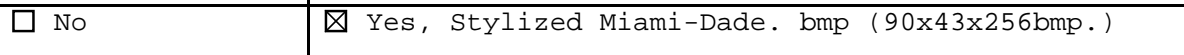 \\
\hline $\begin{array}{l}\text { PROGRAM IS OPEN TO } \\
\text { LABORATORIES : }\end{array}$ & $\begin{array}{ll}\text { All U.S. Private Sector } & \text { All U.S. Government } \\
\bigotimes \text { All Foreign Private Sector } & \text { All Foreign Government } \\
\square \text { Other } & \end{array}$ \\
\hline $\begin{array}{l}\text { AVAILABILITY OF PUBLISHED LIST OF } \\
\text { DIRECTORIES: }\end{array}$ & 囚 Yes, available upon request. \\
\hline $\begin{array}{l}\text { AVAILABILITY OF ASSESSMENT } \\
\text { CRITERIA/PROCEDURES : }\end{array}$ & Available upon request. \\
\hline
\end{tabular}




\begin{tabular}{|c|c|}
\hline $\begin{array}{l}\text { APPROVED/ACCREDITED LABORATORY } \\
\text { MANDATED REQUIREMENTS: }\end{array}$ & 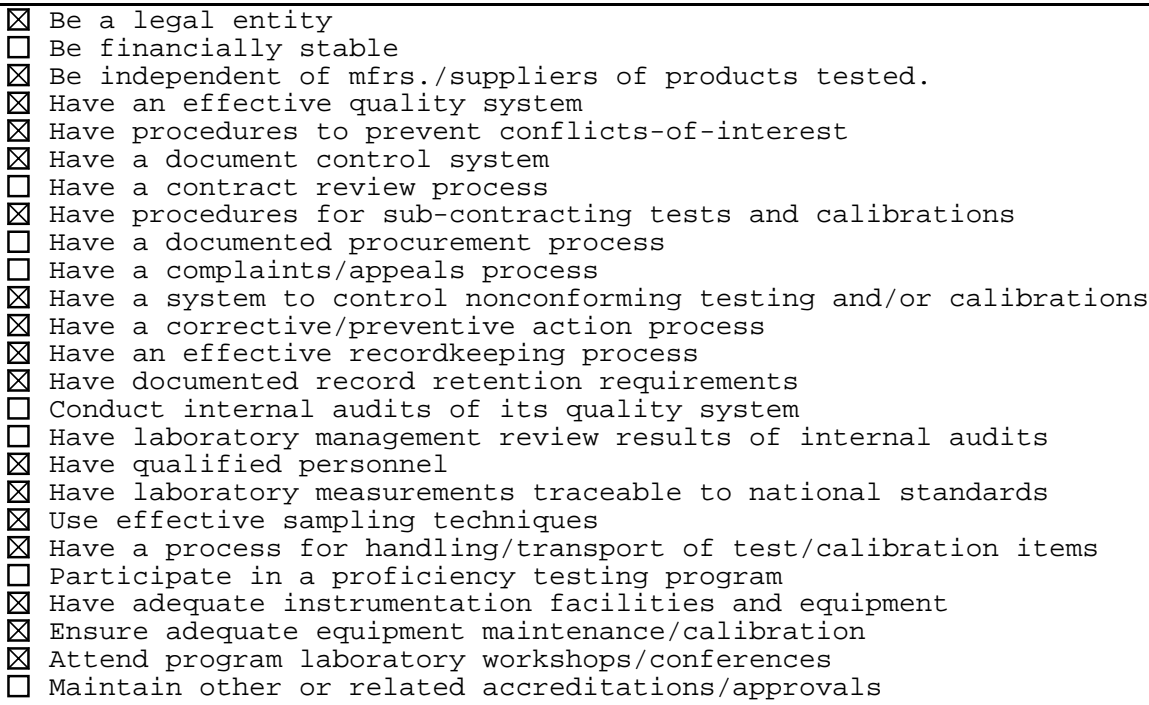 \\
\hline $\begin{array}{l}\text { STANDARD (S) WITH WHICH PROGRAM } \\
\text { CONFORMS: }\end{array}$ & \begin{tabular}{|l|l}
$\square$ No $\square$ Don't know & $\begin{array}{r}\text { Yes, NES (National Evaluation Service, } \\
\text { Inc.), SBCCI (Southern Building } \\
\text { Code Congress, Int'l.). }\end{array}$
\end{tabular} \\
\hline $\begin{array}{l}\text { PROGRAM RECOGNITION/MUTUAL } \\
\text { RECOGNITION ARRANGEMENTS : }\end{array}$ & $\begin{array}{l}\text { Recognized by other countries such as Bahamas, Japan, Dominican } \\
\text { Republic, Germany, Spain, England, Canada, and Mexico. }\end{array}$ \\
\hline
\end{tabular}




\begin{tabular}{|c|c|}
\hline DEPARTMENT/AGENCY : & GEORGIA ENVIRONMENTAL PROTECTION DIVISION \\
\hline ADDRESS : & $\begin{array}{l}\text { Environmental Facilities Assessment Coordinator, } 4220 \text { International } \\
\text { Parkway, Suite 101, Atlanta, GA } 30354\end{array}$ \\
\hline & \begin{tabular}{c|c} 
INTERNET URL: wWW.dnr.state.ga.us/ \\
dnr/environ
\end{tabular} \\
\hline & E-MAIL: ernie-earn@mail.dnr.state.ga.us \\
\hline NAME OF PROGRAM: & Rules for commercial environmental laboratories. \\
\hline PROGRAM DESCRIPTION: & $\begin{array}{l}\text { Legislation required Environmental Protection Division [EPD] to adopt } \\
\text { rules to accredit or approve commercial environmental labs. Did not } \\
\text { provide positions to implement the rules, therefore EPD accredits } \\
\text { laboratories accredited under NELAC program and names third party } \\
\text { accrediting bodies; A2LA, AIHA, NSF, and QAI. }\end{array}$ \\
\hline DATE PROGRAM STARTED: & July 01,2001 \\
\hline FIELDS OF TESTING: & $\begin{array}{ll}\square \text { Acoustic and Vibration Testing } & \square \text { Mechanical Testing } \\
\square \text { Biological Testing } & \square \text { Metrology } \\
\square \text { Chemical Testing } & \square \text { Nondestructive Testing } \\
\square \text { Electrical Testing } & \square \text { Optics and Photometry } \\
\square \text { Ionizing Radiation } & \square \text { Thermal Testing } \\
\text { Other Environmental } & \end{array}$ \\
\hline $\begin{array}{l}\text { CATEGORIES OF PRODUCTS } \\
\text { COVERED BY THE PROGRAM: }\end{array}$ & Environmental samples. \\
\hline $\begin{array}{l}\text { NUMBER OF LABS ACCREDITED/ } \\
\text { APPROVED: }\end{array}$ & Not Applicable. \\
\hline $\begin{array}{l}\text { ACCREDITATION/APPROVAL } \\
\text { VALID FOR: }\end{array}$ & Not Applicable. \\
\hline NUMBER OF ASSESSORS: & None. \\
\hline $\begin{array}{l}\text { MANDATORY ASSESSOR } \\
\text { QUALIFICATIONS : }\end{array}$ & $\square$ Yes \\
\hline PARTICIPATION FEE: & $\square$ Yes \\
\hline FEE SCHEDULE AVAILABLE: & $\square$ Yes \\
\hline PROGRAM HAS AN APPEALS PROCEDURE: & $\square$ Yes, to the accrediting agency. \\
\hline PROGRAM HAS A LOGO: & $\square$ Yes \\
\hline $\begin{array}{l}\text { PROGRAM IS OPEN TO } \\
\text { LABORATORIES : }\end{array}$ & $\begin{array}{llc}\text { All U.S. Private Sector } & \square \text { All U.S. Government } \\
\square \text { All Foreign Private Sector } & \square \text { All Foreign Government } \\
\text { 冈 Other-All commercial environmental laboratories. }\end{array}$ \\
\hline $\begin{array}{l}\text { AVAILABILITY OF PUBLISHED LIST OF } \\
\text { LABORATORIES: }\end{array}$ & $\square$ Yes \\
\hline $\begin{array}{l}\text { AVAILABILITY OF ASSESSMENT } \\
\text { CRITERIA/PROCEDURES: }\end{array}$ & Available from accreditation authorities. \\
\hline
\end{tabular}




\begin{tabular}{|c|c|}
\hline $\begin{array}{l}\text { APPROVED/ACCREDITED LABORATORY } \\
\text { MANDATED REQUIREMENTS: }\end{array}$ & $\begin{array}{l}\text { Be a legal entity } \\
\text { Be financially stable } \\
\text { Be independent of mfrs./suppliers of products tested. } \\
\text { Have an effective quality system } \\
\text { Have procedures to prevent conflicts-of-interest } \\
\text { Have a document control system } \\
\text { Have a contract review process } \\
\text { Have procedures for sub-contracting tests and calibrations } \\
\text { Have a documented procurement process } \\
\text { Have a complaints/appeals process } \\
\text { Have a system to control nonconforming testing and/or calibrations } \\
\text { Have a corrective/preventive action process } \\
\text { Have an effective recordkeeping process } \\
\text { Have documented record retention requirements } \\
\text { H Conduct internal audits of its quality system } \\
\text { Have laboratory management review results of internal audits } \\
\text { Have qualified personnel } \\
\text { Have laboratory measurements traceable to national standards } \\
\text { Hse effective sampling techniques } \\
\bigotimes \text { Have a process for handling/transport of test/calibration items } \\
\text { Participate in a proficiency testing program } \\
\bigotimes \text { Have adequate instrumentation facilities and equipment } \\
\text { Ensure adequate equipment maintenance/calibration } \\
\square \text { Attend program laboratory workshops/conferences } \\
\square \text { Maintain other or related accreditations/approvals }\end{array}$ \\
\hline $\begin{array}{l}\text { STANDARD (S) WITH WHICH PROGRAM } \\
\text { CONFORMS: }\end{array}$ & $\begin{array}{l}\text { Xes, } \\
\text { requirements and labs conform to } \\
\text { Iso } 17025 .\end{array}$ \\
\hline $\begin{array}{l}\text { PROGRAM RECOGNITION/MUTUAL } \\
\text { RECOGNITION ARRANGEMENTS : }\end{array}$ & None. \\
\hline
\end{tabular}




\begin{tabular}{|c|c|}
\hline DEPARTMENT/AGENCY : & $\begin{array}{l}\text { KANSAS-DEPARTMENT OF HEALTH \& ENVIRONMENT-LABORATORY } \\
\text { IMPROVEMENT PROGRAM (\#1) }\end{array}$ \\
\hline \multirow[t]{3}{*}{ ADDRESS : } & Forbes Field, Building 740, Room 2B18, Topeka, KS 66620-0001 \\
\hline & $\begin{array}{l}\text { INTERNET URL: } \\
\text { wWw.state.ks.us/public/kdhe }\end{array}$ \\
\hline & E-MAIL: ssutton@kdhe.state.ks.us \\
\hline NAME OF PROGRAM: & Breath Alcohol Laboratory Program \\
\hline PROGRAM DESCRIPTION: & Accreditation of laboratories that perform breath alcohol testing. \\
\hline \multicolumn{2}{|l|}{ DATE PROGRAM STARTED: } \\
\hline FIELDS OF TESTING: & $\begin{array}{ll}\square \text { Acoustic and Vibration Testing } & \square \text { Mechanical Testing } \\
\bigotimes \text { Biological Testing } & \square \text { Metrology } \\
\square \text { Chemical Testing } & \square \text { Nondestructive Testing } \\
\square \text { Electrical Testing } & \square \text { Optics and Photometry } \\
\square \text { Ionizing Radiation } & \square \text { Thermal Testing } \\
\square \text { Other } & \end{array}$ \\
\hline $\begin{array}{l}\text { CATEGORIES OF PRODUCTS } \\
\text { COVERED BY THE PROGRAM: }\end{array}$ & $\begin{array}{l}\text { Certifies and continuously monitors } 185 \text { evidential breath alcohol } \\
\text { instruments. }\end{array}$ \\
\hline $\begin{array}{l}\text { NUMBER OF LABS ACCREDITED/ } \\
\text { APPROVED: }\end{array}$ & 134 \\
\hline $\begin{array}{l}\text { ACCREDITATION/APPROVAL } \\
\text { VALID FOR: }\end{array}$ & 1 year. \\
\hline NUMBER OF ASSESSORS: & 7 . \\
\hline $\begin{array}{l}\text { MANDATORY ASSESSOR } \\
\text { QUALIFICATIONS : }\end{array}$ & \begin{tabular}{l|l} 
No & $\square$ Yes
\end{tabular} \\
\hline PARTICIPATION FEE: & \begin{tabular}{|l|l|} 
No & $\square$ Yes
\end{tabular} \\
\hline FEE SCHEDULE AVAILABLE: & \begin{tabular}{|l|l|}
$\square$ No & $\square$ Yes
\end{tabular} \\
\hline PROGRAM HAS AN APPEALS PROCEDURE : & \begin{tabular}{|c|c|}
$\square$ No & $\begin{array}{c}\text { Yes, to the Secretary, Department of Health \& } \\
\text { Environment. }\end{array}$
\end{tabular} \\
\hline PROGRAM HAS A LOGO: & Xes, State S \\
\hline $\begin{array}{l}\text { PROGRAM IS OPEN TO } \\
\text { LABORATORIES : }\end{array}$ & $\begin{array}{lll}\text { All U.S. Private Sector } & \square \text { All U.S. Government } \\
\square \text { All Foreign Private Sector } & \square \text { All Foreign Government } \\
\text { Other-Kansas laboratories only } & \end{array}$ \\
\hline $\begin{array}{l}\text { AVAILABILITY OF PUBLISHED LIST OF } \\
\text { LABORATORIES: }\end{array}$ & \begin{tabular}{|l|l}
$\square$ No & $\begin{array}{r}\text { Yes, on the Internet at } \\
\text { www.state.ks.us/public/kdhe. }\end{array}$
\end{tabular} \\
\hline $\begin{array}{l}\text { AVAILABILITY OF ASSESSMENT } \\
\text { CRITERIA/PROCEDURES : }\end{array}$ & Available on request. \\
\hline
\end{tabular}




\begin{tabular}{|c|c|}
\hline $\begin{array}{l}\text { APPROVED/ACCREDITED LABORATORY } \\
\text { MANDATED REQUIREMENTS: }\end{array}$ & $\begin{array}{l}\text { Be a legal entity } \\
\square \text { Be financially stable } \\
\square \text { Be independent of mfrs./suppliers of products tested. } \\
\text { Have an effective quality system } \\
\bigotimes \text { Have procedures to prevent conflicts-of-interest } \\
\square \text { Have a document control system } \\
\square \text { Have a contract review process } \\
\square \text { Have procedures for sub-contracting tests and calibrations } \\
\square \text { Have a documented procurement process } \\
\square \text { Have a complaints/appeals process } \\
\square \text { Have a system to control nonconforming testing and/or calibrations } \\
\square \text { Have a corrective/preventive action process } \\
\bigotimes \text { Have an effective recordkeeping process } \\
\square \text { Have documented record retention requirements } \\
\square \text { Conduct internal audits of its quality system } \\
\bigotimes \text { Have laboratory management review results of internal audits } \\
\bigotimes \text { Have qualified personnel } \\
\square \text { Have laboratory measurements traceable to national standards } \\
\bigotimes \text { Use effective sampling techniques } \\
\square \text { Have a process for handling/transport of test/calibration items } \\
\bigotimes \text { Participate in a proficiency testing program } \\
\square \text { Have adequate instrumentation facilities and equipment } \\
\square \text { Ensure adequate equipment maintenance/calibration } \\
\square \text { Attend program laboratory workshops/conferences } \\
\square \text { Maintain other or related accreditations/approvals }\end{array}$ \\
\hline $\begin{array}{l}\text { STANDARD (S) WITH WHICH PROGRAM } \\
\text { CONFORMS : }\end{array}$ & $\square$ Yes, Federal DOT criteria. \\
\hline $\begin{array}{l}\text { PROGRAM RECOGNITION/MUTUAL } \\
\text { RECOGNITION ARRANGEMENTS : }\end{array}$ & None. \\
\hline
\end{tabular}




\begin{tabular}{|c|c|}
\hline DEPARTMENT/AGENCY : & $\begin{array}{l}\text { KANSAS-DEPARTMENT OF HEALTH \& ENVIRONMENT-LABORATORY } \\
\text { IMPROVEMENT PROGRAM (\#2) }\end{array}$ \\
\hline \multirow[t]{3}{*}{ ADDRESS : } & Forbes Field, Building 740, Room 2B18, Topeka, KS 66620-0001 \\
\hline & \begin{tabular}{|l|c|} 
PHONE : 785-296-1640 & $\begin{array}{c}\text { INTERNET URL: www.state.ks.us/public/ } \\
\text { kdhe }\end{array}$
\end{tabular} \\
\hline & \begin{tabular}{|l|l|} 
FAX: $785-296-8068$ & E-MAIL: ssutton@kdhe.state.ks.us
\end{tabular} \\
\hline NAME OF PROGRAM: & $\begin{array}{l}\text { Environmental Laboratory Improvement Program-Laboratory Certification } \\
\text { (Accreditation) }\end{array}$ \\
\hline PROGRAM DESCRIPTION: & $\begin{array}{l}\text { Accreditation of laboratories that perform laboratory testing and } \\
\text { analysis for environmental regulations-National Environmental } \\
\text { Laboratory Accreditation Program. }\end{array}$ \\
\hline \multicolumn{2}{|l|}{ DATE PROGRAM STARTED: } \\
\hline FIELDS OF TESTING: & 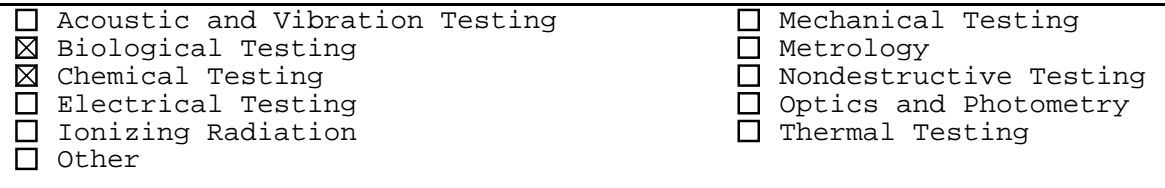 \\
\hline $\begin{array}{l}\text { CATEGORIES OF PRODUCTS } \\
\text { COVERED BY THE PROGRAM: }\end{array}$ & Air, soil and water. \\
\hline $\begin{array}{l}\text { NUMBER OF LABS ACCREDITED/ } \\
\text { APPROVED: }\end{array}$ & 377 \\
\hline $\begin{array}{l}\text { ACCREDITATION/APPROVAL } \\
\text { VALID FOR: }\end{array}$ & 2 years. \\
\hline NUMBER OF ASSESSORS: & 7. \\
\hline $\begin{array}{l}\text { MANDATORY ASSESSOR } \\
\text { QUALIFICATIONS : }\end{array}$ & \begin{tabular}{|c|c|}
$\square$ No & $\begin{array}{c}\text { Yes, requirements of the National Environmental } \\
\text { Laboratory Accreditation Program. }\end{array}$ \\
\end{tabular} \\
\hline PARTICIPATION FEE: & 凶Yes \\
\hline FEE SCHEDULE AVAILABLE: & $\begin{array}{l}\text { Q Yes, on the Internet at } \\
\text { www.state.ks.us/public/kdhe. }\end{array}$ \\
\hline PROGRAM HAS AN APPEALS PROCEDURE: & $\begin{array}{l}\text { 囚 Yes, to the Secretary, Department of Health \& } \\
\text { Environment. }\end{array}$ \\
\hline PROGRAM HAS A LOGO: & $\nabla$ Yes, State Seal. \\
\hline $\begin{array}{l}\text { PROGRAM IS OPEN TO } \\
\text { LABORATORIES : }\end{array}$ & $\begin{array}{ll}\text { All U.S. Private Sector } & \text { All U.S. Government } \\
\text { All Foreign Private Sector } & \text { All Foreign Government } \\
\square \text { Other } & \end{array}$ \\
\hline $\begin{array}{l}\text { AVAILABILITY OF PUBLISHED LIST OF } \\
\text { LABORATORIES: }\end{array}$ & $\begin{array}{r}\text { 冈 Yes, on the Internet at } \\
\text { www.state.ks.us/public/kdhe }\end{array}$ \\
\hline $\begin{array}{l}\text { AVAILABILITY OF ASSESSMENT } \\
\text { CRITERIA/PROCEDURES : }\end{array}$ & Available upon request. \\
\hline
\end{tabular}




\begin{tabular}{|c|c|}
\hline $\begin{array}{l}\text { APPROVED/ACCREDITED LABORATORY } \\
\text { MANDATED REQUIREMENTS: }\end{array}$ & 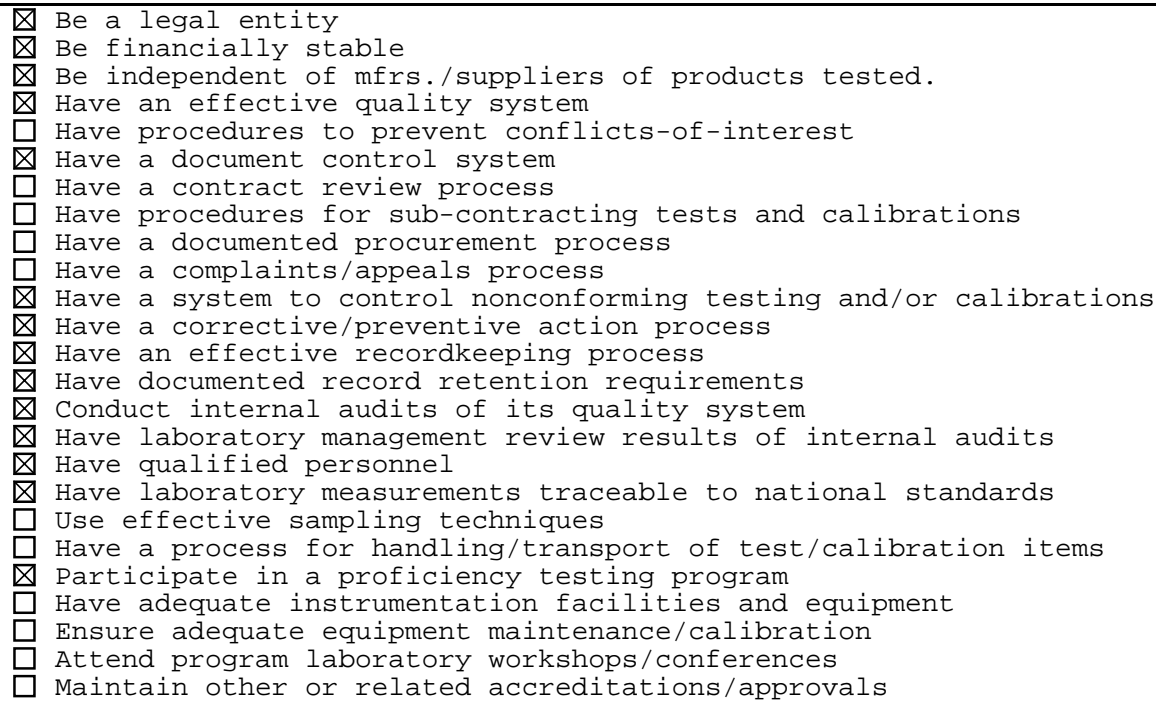 \\
\hline $\begin{array}{l}\text { STANDARD ( } S \text { ) WITH WHICH PROGRAM } \\
\text { CONFORMS : }\end{array}$ & \begin{tabular}{|l|r|r|}
$\square$ No $\square$ Don't know & $\begin{array}{r}\text { Yequirements of the National } \\
\text { Environmental Laboratory } \\
\text { Accreditation Program [NELAP] }\end{array}$
\end{tabular} \\
\hline $\begin{array}{l}\text { PROGRAM RECOGNITION/MUTUAL } \\
\text { RECOGNITION ARRANGEMENTS: }\end{array}$ & $\mathrm{N}$ \\
\hline
\end{tabular}




\begin{tabular}{|c|c|}
\hline DEPARTMENT/AGENCY : & $\begin{array}{l}\text { KANSAS-DEPARTMENT OF HEALTH \& ENVIRONMENT-LABORATORY } \\
\text { IMPROVEMENT PROGRAM (\#3) }\end{array}$ \\
\hline \multirow[t]{3}{*}{ ADDRESS : } & Forbes Field, Building 740, Room 2B18, Topeka, KS 66620-0001 \\
\hline & \begin{tabular}{l|l} 
PHONE : 785-296-1640 & INTERNET URL: www.state.ks.us/public/kdhe
\end{tabular} \\
\hline & E-MAIL: ssutton@kdhe.state.ks.us \\
\hline NAME OF PROGRAM: & Clinical Laboratory Accreditation Program \\
\hline PROGRAM DESCRIPTION: & Accreditation of commercial public health laboratories. \\
\hline \multicolumn{2}{|l|}{ DATE PROGRAM STARTED: } \\
\hline FIELDS OF TESTING: & $\begin{array}{ll}\text { Acoustic and Vibration Testing } & \square \text { Mechanical Testing } \\
\square \text { Biological Testing } & \square \text { Metrology } \\
\square \text { Chemical Testing } & \square \text { Nondestructive Testing } \\
\square \text { Electrical Testing } & \square \text { Optics and Photometry } \\
\square \text { Ionizing Radiation } & \square \text { Thermal Testing } \\
\square \text { Other } & \end{array}$ \\
\hline $\begin{array}{l}\text { CATEGORIES OF PRODUCTS } \\
\text { COVERED BY THE PROGRAM: }\end{array}$ & Human body matter. \\
\hline $\begin{array}{l}\text { NUMBER OF LABS ACCREDITED/ } \\
\text { APPROVED: }\end{array}$ & 2,234 \\
\hline $\begin{array}{l}\text { ACCREDITATION/APPROVAL } \\
\text { VALID FOR: }\end{array}$ & 2 years. \\
\hline NUMBER OF ASSESSORS: & 7. \\
\hline $\begin{array}{l}\text { MANDATORY ASSESSOR } \\
\text { QUALIFICATIONS : }\end{array}$ & 区 Yes, must be medical technicians. \\
\hline PARTICIPATION FEE: & $\square$ Yes, available upon request. \\
\hline FEE SCHEDULE AVAILABLE: & \ Yes, available upon request \\
\hline $\begin{array}{l}\text { PROGRAM HAS AN APPEALS } \\
\text { PROCEDURE : }\end{array}$ & $\begin{array}{l}\text { \ Yes, to the Secretary, Department of Health \& } \\
\text { Environment. }\end{array}$ \\
\hline PROGRAM HAS A LOGO: & 区 Yes, State Seal. \\
\hline $\begin{array}{l}\text { PROGRAM IS OPEN TO } \\
\text { LABORATORIES : }\end{array}$ & $\begin{array}{lll}\text { All U.S. Private Sector } & \square \text { All U.S. Government } \\
\square \text { All Foreign Private Sector } & \square \text { All Foreign Government } \\
\square \text { Other } & \end{array}$ \\
\hline $\begin{array}{l}\text { AVAILABILITY OF PUBLISHED LIST OF } \\
\text { LABORATORIES : }\end{array}$ & $\begin{array}{c}\text { Yes, on the Internet at } \\
\text { www.state.ks.us/public/kdhe. }\end{array}$ \\
\hline $\begin{array}{l}\text { AVAILABILITY OF ASSESSMENT } \\
\text { CRITERIA/PROCEDURES: }\end{array}$ & $\begin{array}{l}\text { Federal Health Care Finance Administration [HCFA] Code of Federal } \\
\text { Regulations. }\end{array}$ \\
\hline
\end{tabular}




\begin{tabular}{|c|c|}
\hline $\begin{array}{l}\text { APPROVED/ACCREDITED LABORATORY } \\
\text { MANDATED REQUIREMENTS: }\end{array}$ & 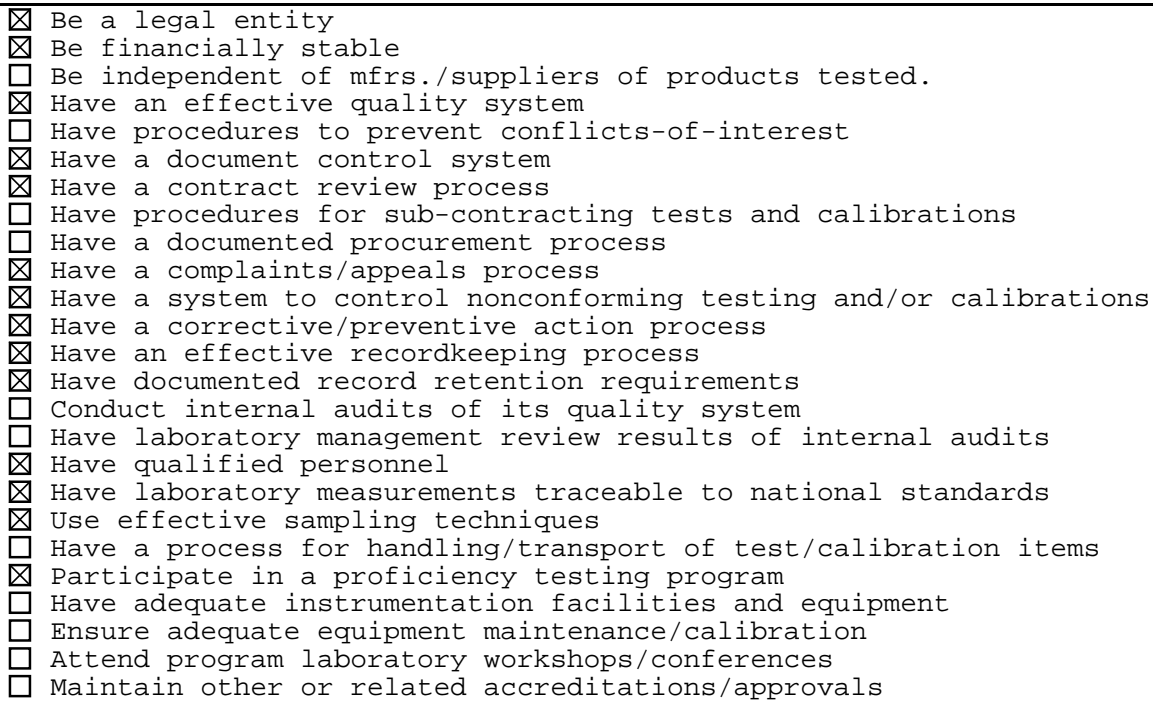 \\
\hline $\begin{array}{l}\text { STANDARD (S) WITH WHICH PROGRAM } \\
\text { CONFORMS : }\end{array}$ & $\triangle$ Yes, those of HCFA. \\
\hline $\begin{array}{l}\text { PROGRAM RECOGNITION/MUTUAL } \\
\text { RECOGNITION ARRANGEMENTS : }\end{array}$ & Nor \\
\hline
\end{tabular}




\begin{tabular}{|c|c|}
\hline DEPARTMENT/AGENCY: & $\begin{array}{l}\text { KANSAS-DEPARTMENT OF HEALTH \& ENVIRONMENT-LABORATORY } \\
\text { IMPROVEMENT PROGRAM }(\# 4)\end{array}$ \\
\hline ADDRESS : & Forbes Field, Building 740, Room 2B18, Topeka, KS 66620-0001 \\
\hline & $\begin{array}{c}\text { INTERNET URL: wWw.state.ks.us/ } \\
\text { public/kdhe }\end{array}$ \\
\hline & E-MAIL: ssutton@kdhe.state.ks.us \\
\hline NAME OF PROGRAM: & Approved Drug Testing Laboratories \\
\hline PROGRAM DESCRIPTION: & $\begin{array}{l}\text { Accreditation of laboratories that perform laboratory testing and } \\
\text { analysis for drugs of abuse. }\end{array}$ \\
\hline DATE PROGRAM STARTED: & Not available. \\
\hline FIELDS OF TESTING: & $\begin{array}{ll}\square \text { Acoustic and Vibration Testing } & \square \text { Mechanical Testing } \\
\text { Biological Testing } & \square \text { Metrology } \\
\text { B Chemical Testing } & \square \text { Nondestructive Testing } \\
\square \text { Electrical Testing } & \square \text { Optics and Photometry } \\
\square \text { Ionizing Radiation } & \square \text { Thermal Testing } \\
\square \text { Other } & \end{array}$ \\
\hline $\begin{array}{l}\text { CATEGORIES OF PRODUCTS } \\
\text { COVERED BY THE PROGRAM: }\end{array}$ & Drugs of abuse. \\
\hline $\begin{array}{l}\text { NUMBER OF LABS ACCREDITED/ } \\
\text { APPROVED: }\end{array}$ & 46. \\
\hline $\begin{array}{l}\text { ACCREDITATION/APPROVAL } \\
\text { VALID FOR: }\end{array}$ & 2 years. \\
\hline NUMBER OF ASSESSORS: & 7 . \\
\hline $\begin{array}{l}\text { MANDATORY ASSESSOR } \\
\text { QUALIFICATIONS : }\end{array}$ & $\square$ Yes \\
\hline PARTICIPATION FEE : & $\square$ Yes \\
\hline FEE SCHEDULE AVAILABLE: & $\square$ Yes \\
\hline $\begin{array}{l}\text { PROGRAM HAS AN APPEALS } \\
\text { PROCEDURE : }\end{array}$ & $\begin{array}{l}\otimes \text { Yes, to the Secretary, Department of Health \& } \\
\text { Environment. }\end{array}$ \\
\hline PROGRAM HAS A LOGO: & Q Yes, State Seal \\
\hline $\begin{array}{l}\text { PROGRAM IS OPEN TO } \\
\text { LABORATORIES : }\end{array}$ & $\begin{array}{ll}\text { All U.S. Private Sector } & \text { All U.S. Government } \\
\text { All Foreign Private Sector } & \text { All Foreign Government } \\
\square \text { Other } & \end{array}$ \\
\hline $\begin{array}{l}\text { AVAILABILITY OF PUBLISHED LIST OF } \\
\text { LABORATORIES : }\end{array}$ & $\begin{aligned} & \text { Yes, on the Internet at } \\
& \text { www.state.ks.us/public/kdhe }\end{aligned}$ \\
\hline $\begin{array}{l}\text { AVAILABILITY OF ASSESSMENT } \\
\text { CRITERIA/PROCEDURES : }\end{array}$ & Available upon request. \\
\hline
\end{tabular}




\begin{tabular}{|c|c|}
\hline $\begin{array}{l}\text { APPROVED/ACCREDITED LABORATORY } \\
\text { MANDATED REQUIREMENTS: }\end{array}$ & 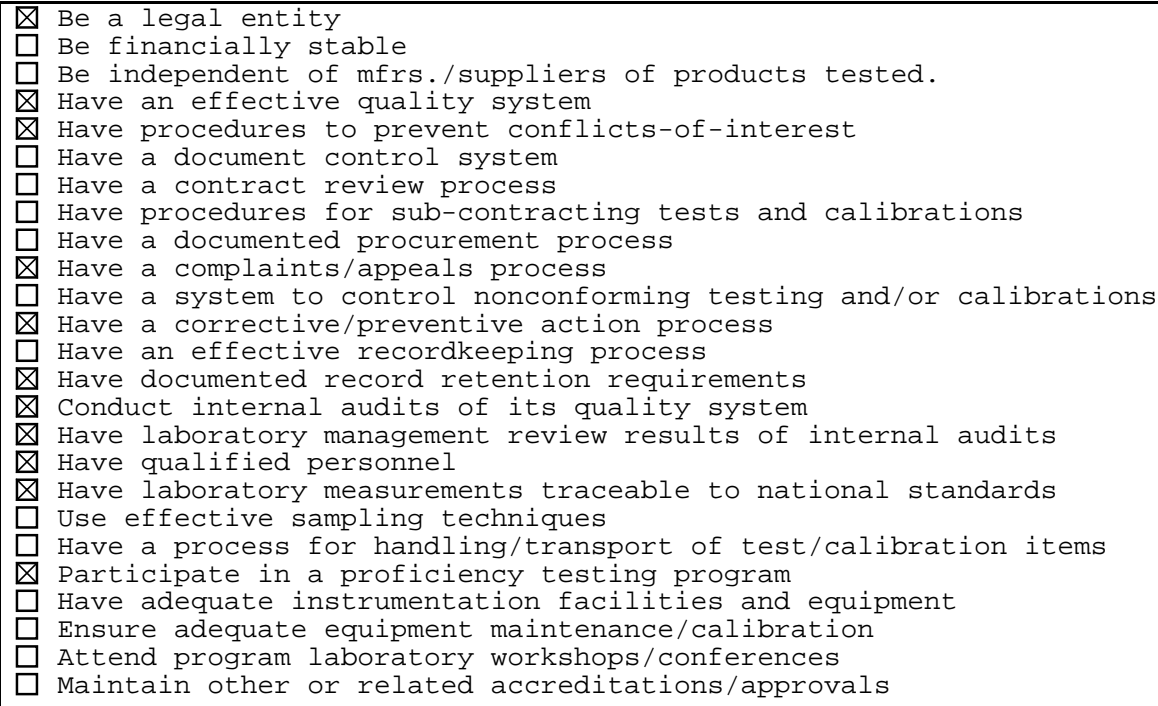 \\
\hline $\begin{array}{l}\text { STANDARD (S) WITH WHICH PROGRAM } \\
\text { CONFORMS : }\end{array}$ & $\begin{array}{l}\text { Yes, Requirements of the Substance } \\
\text { Abuse \& Mental Health Services } \\
\text { Administration [SAMHSA] of U.S. } \\
\text { Dept of Health \& Human Services. }\end{array}$ \\
\hline $\begin{array}{l}\text { PROGRAM RECOGNITION/MUTUAL } \\
\text { RECOGNITION ARRANGEMENTS : }\end{array}$ & SAMHSA. \\
\hline
\end{tabular}


DATE RECEIVED: 02/01

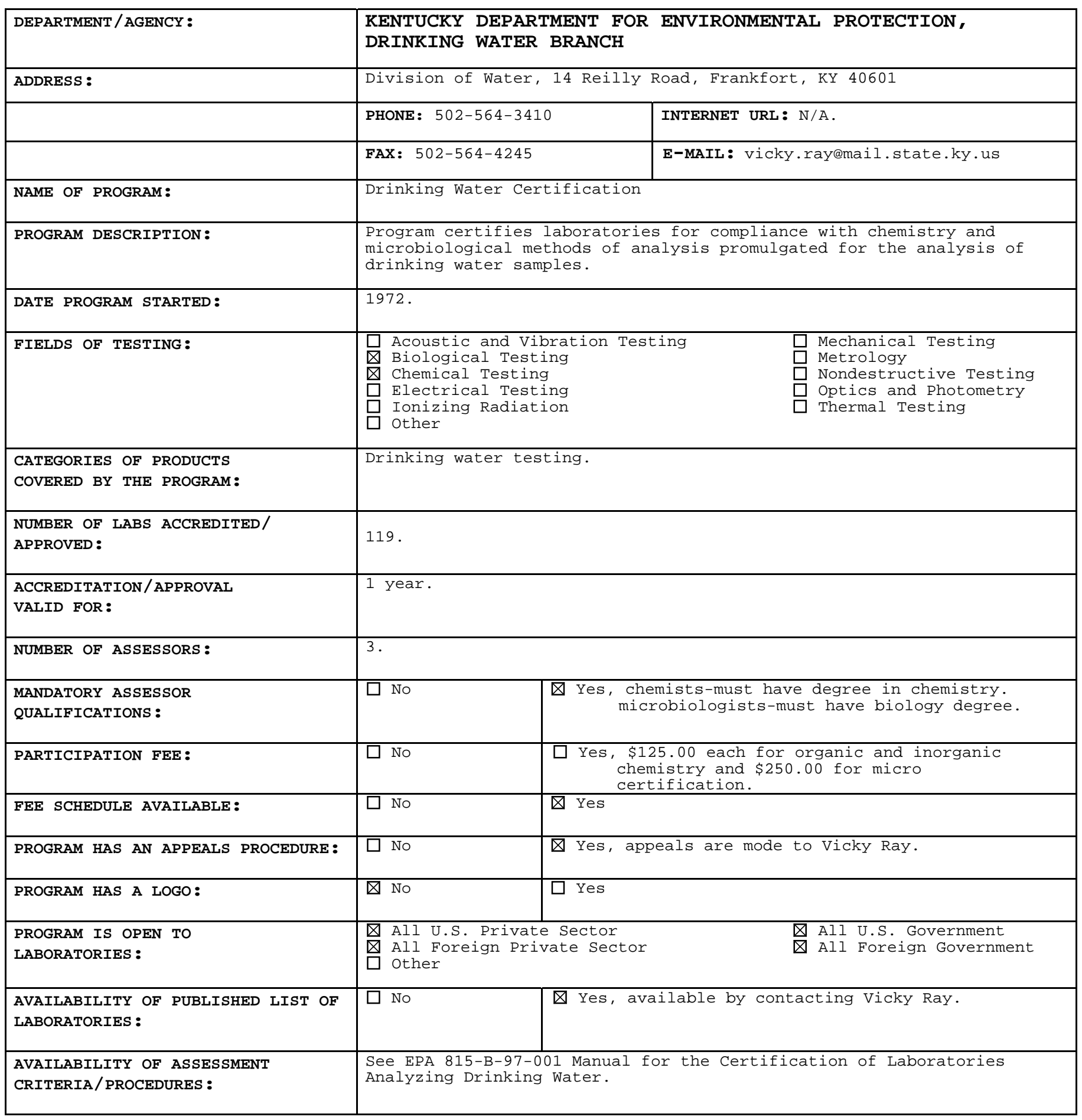




\begin{tabular}{|c|c|}
\hline $\begin{array}{l}\text { APPROVED/ACCREDITED LABORATORY } \\
\text { MANDATED REQUIREMENTS: }\end{array}$ & 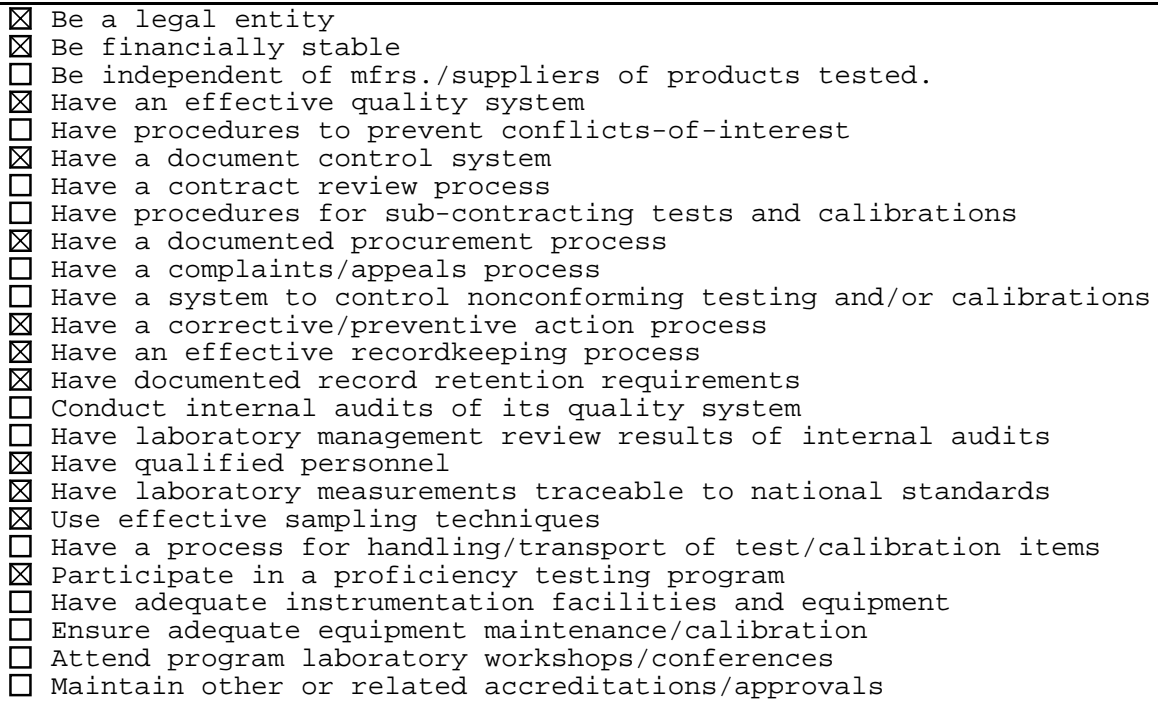 \\
\hline $\begin{array}{l}\text { STANDARD (S) WITH WHICH PROGRAM } \\
\text { CONFORMS : }\end{array}$ & $\square$ No $\square$ Don't know \\
\hline $\begin{array}{l}\text { PROGRAM RECOGNITION/MUTUAL } \\
\text { RECOGNITION ARRANGEMENTS : }\end{array}$ & Not applicable. \\
\hline
\end{tabular}




\begin{tabular}{|c|c|}
\hline DEPARTMENT/AGENCY : & LOUISIANA DEPARTMENT OF ENVIRONMENTAL QUALITY \\
\hline ADDRESS : & $\begin{array}{l}\text { Administrator Laboratory Services Division, Louisiana DEQ, P.O. Box } \\
82231 \text {, Baton Rouge, LA } 70884-2231\end{array}$ \\
\hline & $\begin{array}{c}\text { INTERNET URL: www, deq, state, la.us / } \\
\text { laboratory }\end{array}$ \\
\hline & E-MAIL: louis-j@deq.state.la.us \\
\hline NAME OF PROGRAM: & Louisiana Environmental Laboratory Accreditation Program \\
\hline PROGRAM DESCRIPTION: & $\begin{array}{l}\text { Regulations provide requirements for an accreditation program } \\
\text { specifically applicable to commercial laboratories that provide } \\
\text { chemical analysis, analytical results, or other test data to the } \\
\text { department, by contract or by agreement. The program is designed to } \\
\text { ensure that the laboratory is capable of generating accurate, } \\
\text { reliable data, using department approved methods. Ref.Title } 33 \text { Part I } \\
\text { subpart3:4501. }\end{array}$ \\
\hline DATE PROGRAM STARTED : & November 1998. \\
\hline FIELDS OF TESTING: & $\begin{array}{ll}\square \text { Acoustic and Vibration Testing } & \square \text { Mechanical Testing } \\
\bigotimes \text { Biological Testing } & \square \text { Metrology } \\
\bigotimes \text { Chemical Testing } & \square \text { Nondestructive Testing } \\
\square \text { Electrical Testing } & \square \text { Optics and Photometry } \\
\square \text { Ionizing Radiation } & \square \text { Thermal Testing } \\
\square \text { Other } & \end{array}$ \\
\hline $\begin{array}{l}\text { CATEGORIES OF PRODUCTS } \\
\text { COVERED BY THE PROGRAM: }\end{array}$ & Environmental laboratory data. \\
\hline $\begin{array}{l}\text { NUMBER OF LABS ACCREDITED/ } \\
\text { APPROVED : }\end{array}$ & 45 with 75 more in final stages of accreditation. \\
\hline $\begin{array}{l}\text { ACCREDITATION/APPROVAL } \\
\text { VALID FOR: }\end{array}$ & 1 year. \\
\hline NUMBER OF ASSESSORS: & 5, plus have contract with company with 4 assessors. \\
\hline $\begin{array}{l}\text { MANDATORY ASSESSOR } \\
\text { QUALIFICATIONS : }\end{array}$ & X Yes, NELAC requirements are followed. \\
\hline PARTICIPATION FEE: & $\begin{array}{l}\text { Yes, on the website at } \\
\text { www. deq. state.la.us/laboratory/instructions } \\
\text { and application form. }\end{array}$ \\
\hline FEE SCHEDULE AVAILABLE: & $\begin{array}{l}\text { Q Yes, on the website at } \\
\text { www.deq. state.la.us/laboratory/instructions } \\
\text { and application form. }\end{array}$ \\
\hline PROGRAM HAS AN APPEALS PROCEDURE: & 区 Yes, Secretary of DEQ, State of Louisiana. \\
\hline PROGRAM HAS A LOGO: & \begin{tabular}{|l|l}
$\square$ No & Yes, concentric rectangles with "LELAP' in \\
& center and "Louisiana Environmental \\
Laboratory" on top and "Accreditation \\
Program" on bottom.
\end{tabular} \\
\hline $\begin{array}{l}\text { PROGRAM IS OPEN TO } \\
\text { LABORATORIES : }\end{array}$ & $\begin{array}{ll}\text { Мll U.S. Private Sector } & \square \text { All U.S. Government } \\
\text { \ All Foreign Private Sector } & \square \text { All Foreign Government } \\
\square \text { Other } & \end{array}$ \\
\hline $\begin{array}{l}\text { AVAILABILITY OF PUBLISHED LIST OF } \\
\text { LABORATORIES: }\end{array}$ & $\begin{array}{l}\text { 区 Yes, on website at } \\
\text { www.deq.state.la.us/laboratory. }\end{array}$ \\
\hline $\begin{array}{l}\text { AVAILABILITY OF ASSESSMENT } \\
\text { CRITERIA/PROCEDURES: }\end{array}$ & Title 33 Part I subpart 3 on website URL. \\
\hline
\end{tabular}




\begin{tabular}{|c|c|}
\hline $\begin{array}{l}\text { APPROVED/ACCREDITED LABORATORY } \\
\text { MANDATED REQUIREMENTS: }\end{array}$ & $\begin{array}{l}\text { Be a legal entity } \\
\text { Be financially stable } \\
\text { Be independent of mfrs./suppliers of products tested. } \\
\text { Have an effective quality system } \\
\text { Have procedures to prevent conflicts-of-interest } \\
\text { Have a document control system } \\
\text { Have a contract review process } \\
\text { Have procedures for sub-contracting tests and calibrations } \\
\text { Have a documented procurement process } \\
\text { Have a complaints/appeals process } \\
\text { Have a system to control nonconforming testing and/or calibrations } \\
\text { Have a corrective/preventive action process } \\
\text { Have an effective recordkeeping process } \\
\text { Have documented record retention requirements } \\
\text { H Conduct internal audits of its quality system } \\
\text { Have laboratory management review results of internal audits } \\
\text { Have qualified personnel } \\
\text { Have laboratory measurements traceable to national standards } \\
\text { Use effective sampling techniques } \\
\bigotimes \text { Have a process for handling/transport of test/calibration items } \\
\bigotimes \text { Participate in a proficiency testing program } \\
\bigotimes \text { Have adequate instrumentation facilities and equipment } \\
\text { Ensure adequate equipment maintenance/calibration } \\
\square \text { Attend program laboratory workshops/conferences } \\
\square \text { Maintain other or related accreditations/approvals }\end{array}$ \\
\hline $\begin{array}{l}\text { STANDARD (S) WITH WHICH PROGRAM } \\
\text { CONFORMS: }\end{array}$ & $\triangle$ Yes, those of NELAC. \\
\hline $\begin{array}{l}\text { PROGRAM RECOGNITION/MUTUAL } \\
\text { RECOGNITION ARRANGEMENTS : }\end{array}$ & In process of becoming a NELAP Accrediting Authority. \\
\hline
\end{tabular}




\begin{tabular}{|c|c|}
\hline DEPARTMENT/AGENCY : & $\begin{array}{l}\text { LOUISIANA DEPARTMENT OF HEALTH \& HOSPITALS, OFFICE OF } \\
\text { PUBLIC HEALTH }\end{array}$ \\
\hline ADDRESS : & $\begin{array}{l}\text { Public Health Laboratory Director, } 325 \text { Loyola Avenue, Room 709, New } \\
\text { Orleans, LA } 70112\end{array}$ \\
\hline & PHONE : $504-568-5375$ \\
\hline & E-MAIL: HBradforeDHH.state.LA.US \\
\hline NAME OF PROGRAM: & $\begin{array}{l}\text { Louisiana Department of Health \& Hospitals Laboratory Accreditation } \\
\text { Program }\end{array}$ \\
\hline PROGRAM DESCRIPTION: & Accredit laboratories to perform the analysis of drinking water. \\
\hline DATE PROGRAM STARTED: & 1989. \\
\hline FIELDS OF TESTING: & $\begin{array}{ll}\square \text { Acoustic and Vibration Testing } & \square \text { Mechanical Testing } \\
\bigotimes \text { Biological Testing } & \square \text { Metrology } \\
\bigotimes \text { Chemical Testing } & \square \text { Nondestructive Testing } \\
\square \text { Electrical Testing } & \square \text { Optics and Photometry } \\
\square \text { Ionizing Radiation } & \square \text { Thermal Testing } \\
\square \text { Other } & \end{array}$ \\
\hline $\begin{array}{l}\text { CATEGORIES OF PRODUCTS } \\
\text { COVERED BY THE PROGRAM: }\end{array}$ & Drinking water. \\
\hline $\begin{array}{l}\text { NUMBER OF LABS ACCREDITED/ } \\
\text { APPROVED: }\end{array}$ & 26. \\
\hline $\begin{array}{l}\text { ACCREDITATION/APPROVAL } \\
\text { VALID FOR: }\end{array}$ & Chemistry for one year; microbiological for two years. \\
\hline NUMBER OF ASSESSORS: & 4 \\
\hline $\begin{array}{l}\text { MANDATORY ASSESSOR } \\
\text { QUALIFICATIONS : }\end{array}$ & \begin{tabular}{c|c} 
No & $\begin{array}{c}\text { Yes, must have a B.S.degree and pass EPA } \\
\text { training courses. }\end{array}$
\end{tabular} \\
\hline PARTICIPATION FEE: & $\begin{array}{l}\text { 区 Yes, Chemistry-Organic Parameters }-\$ 800.00, \\
\text { Inorganic Parameters } \$ 750.00, \text { Both Organic } \\
\text { \& Inorganic Parameters } \$ 1000.00, \\
\text { Radiological Parameters } \$ 800.00, \\
\text { Microbiological Paramenters }-\$ 500.00 .\end{array}$ \\
\hline FEE SCHEDULE AVAILABLE: & $\square$ Yes, schedule is listed above. \\
\hline PROGRAM HAS AN APPEALS PROCEDURE: & 囚 Yes, to an administrative law judge. \\
\hline PROGRAM HAS A LOGO: & 区 Yes, Gold stamp with the seal of the department. \\
\hline $\begin{array}{l}\text { PROGRAM IS OPEN TO } \\
\text { LABORATORIES : }\end{array}$ & $\begin{array}{ll}\text { All U.S. Private Sector } & \text { All U.S. Government } \\
\square \text { All Foreign Private Sector } & \square \text { All Foreign Government } \\
\square \text { Other } & \end{array}$ \\
\hline $\begin{array}{l}\text { AVAILABILITY OF PUBLISHED LIST OF } \\
\text { LABORATORIES: }\end{array}$ & \ Yes, list is available upon request. \\
\hline $\begin{array}{l}\text { AVAILABILITY OF ASSESSMENT } \\
\text { CRITERIA/PROCEDURES : }\end{array}$ & Available upon request. \\
\hline
\end{tabular}




\begin{tabular}{|c|c|}
\hline $\begin{array}{l}\text { APPROVED/ACCREDITED LABORATORY } \\
\text { MANDATED REQUIREMENTS: }\end{array}$ & $\begin{array}{l}\text { Be a legal entity } \\
\square \text { Be financially stable } \\
\text { Be independent of mfrs./suppliers of products tested. } \\
\text { Have an effective quality system } \\
\text { Have procedures to prevent conflicts-of-interest } \\
\square \text { Have a document control system } \\
\square \text { Have a contract review process } \\
\text { Have procedures for sub-contracting tests and calibrations } \\
\square \text { Have a documented procurement process } \\
\text { Have a complaints/appeals process } \\
\text { Have a system to control nonconforming testing and/or calibrations } \\
\text { Have a corrective/preventive action process } \\
\text { Have an effective recordkeeping process } \\
\text { Have documented record retention requirements } \\
\text { H Conduct internal audits of its quality system } \\
\text { Have laboratory management review results of internal audits } \\
\text { Have qualified personnel } \\
\text { Have laboratory measurements traceable to national standards } \\
\text { Hse effective sampling techniques } \\
\square \text { Have a process for handling/transport of test/calibration items } \\
\bigotimes \text { Participate in a proficiency testing program } \\
\bigotimes \text { Have adequate instrumentation facilities and equipment } \\
\text { Ensure adequate equipment maintenance/calibration } \\
\square \text { Attend program laboratory workshops/conferences } \\
\square \text { Maintain other or related accreditations/approvals }\end{array}$ \\
\hline $\begin{array}{l}\text { STANDARD (S) WITH WHICH PROGRAM } \\
\text { CONEORMS: }\end{array}$ & $\begin{array}{l}\otimes \text { Yes, requirements of the National } \\
\text { Environmental Laboratory } \\
\text { Accreditation Conference [NELAC]. }\end{array}$ \\
\hline $\begin{array}{l}\text { PROGRAM RECOGNITION/MUTUAL } \\
\text { RECOGNITION ARRANGEMENTS : }\end{array}$ & nized by National Environmental Laboratory Program [NELAP]. \\
\hline
\end{tabular}




\begin{tabular}{|c|c|}
\hline DEPARTMENT/AGENCY : & $\begin{array}{l}\text { MINNESOTA DEPARTMENT OF AGRICULTURE, AGRONOMY/PLANT } \\
\text { PROTECTION INCIDENT RESPONSE PROGRAM }\end{array}$ \\
\hline ADDRESS : & 90 West Plato Blvd., St. Paul, MN 55107 \\
\hline & $\begin{array}{l}\text { INTERNET URL: } \\
\text { http://www.mda.state.mn.us }\end{array}$ \\
\hline & FAX: $\quad 651-297-2271$ \\
\hline NAME OF PROGRAM: & Incident Response Program \\
\hline PROGRAM DESCRIPTION: & To investigate and remediate agricultural chemical release sites. \\
\hline DATE PROGRAM STARTED: & 1989. \\
\hline FIELDS OF TESTING: & $\begin{array}{ll}\square \text { Acoustic and Vibration Testing } & \square \text { Mechanical Testing } \\
\square \text { Biological Testing } & \square \text { Metrology } \\
\bigotimes \text { Chemical Testing } & \square \text { Nondestructive Testing } \\
\square \text { Electrical Testing } & \square \text { Optics and Photometry } \\
\square \text { Ionizing Radiation } & \square \text { Thermal Testing } \\
\square \text { Other } & \end{array}$ \\
\hline $\begin{array}{l}\text { CATEGORIES OF PRODUCTS } \\
\text { COVERED BY THE PROGRAM: }\end{array}$ & Pesticide residues in soil and water. \\
\hline $\begin{array}{l}\text { NUMBER OF LABS ACCREDITED/ } \\
\text { APPROVED: }\end{array}$ & 6 labs. \\
\hline $\begin{array}{l}\text { ACCREDITATION/APPROVAL } \\
\text { VALID FOR: }\end{array}$ & 1 year. \\
\hline NUMBER OF ASSESSORS: & 4. \\
\hline $\begin{array}{l}\text { MANDATORY ASSESSOR } \\
\text { QUALIFICATIONS : }\end{array}$ & \begin{tabular}{c|c}
$\square$ No & $\begin{array}{c}\text { Yes. only for chemical staff that analyze } \\
\text { pesticides. }\end{array}$
\end{tabular} \\
\hline PARTICIPATION FEE: & $\square$ Yes \\
\hline FEE SCHEDULE AVAILABLE: & $\square$ Yes \\
\hline PROGRAM HAS AN APPEALS PROCEDURE : & $\square$ Yes \\
\hline PROGRAM HAS A LOGO: & $\square$ Yes \\
\hline $\begin{array}{l}\text { PROGRAM IS OPEN TO } \\
\text { LABORATORIES : }\end{array}$ & $\begin{array}{ll}\text { All U.S. Private Sector } & \square \text { All U.S. Government } \\
\square \text { All Foreign Private Sector } & \square \text { All Foreign Government } \\
\square \text { Other } & \end{array}$ \\
\hline $\begin{array}{l}\text { AVAILABILITY OF PUBLISHED LIST OF } \\
\text { DIRECTORIES AVAILABLE: }\end{array}$ & \begin{tabular}{|l} 
Yes, Guidance Document 23: Pre-approved \\
Commercial Laboratories: Fixed Base and \\
Mobile; \\
Guidance Document 24: Fixed Base \\
Laboratory Quality Assurance/Quality \\
Control Plans; \\
Guidance Document 25: Mobile Laboratory \\
Quality Assurance/Quality Control Plans; \\
Guidance Document 26: Analytical Lists \\
for Pesticide Incident Investigations.
\end{tabular} \\
\hline $\begin{array}{l}\text { AVAILABILITY OF ASSESSMENT } \\
\text { CRITERIA/PROCEDURES : }\end{array}$ & See published list of directories above. \\
\hline
\end{tabular}




\begin{tabular}{|c|c|}
\hline $\begin{array}{l}\text { APPROVED/ACCREDITED LABORATORY } \\
\text { MANDATED REQUIREMENTS: }\end{array}$ & 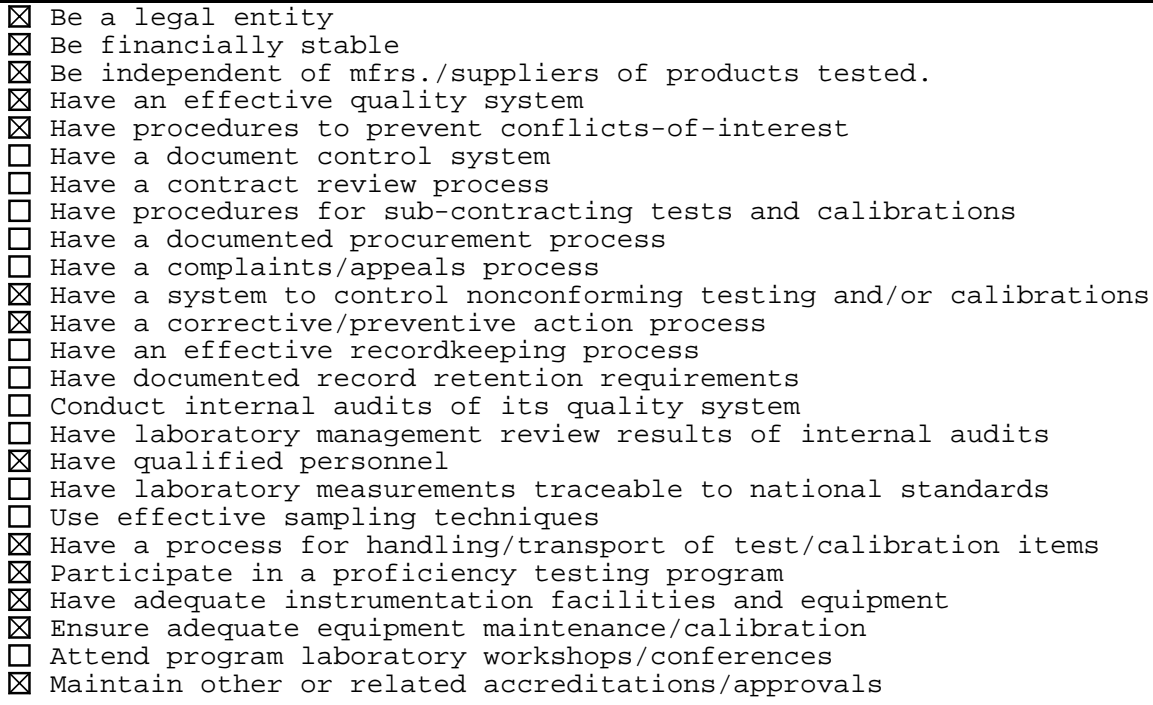 \\
\hline $\begin{array}{l}\text { STANDARD (S) WITH WHICH PROGRAM } \\
\text { CONFORMS: }\end{array}$ & $\square$ No $\quad$ Don't know \\
\hline $\begin{array}{l}\text { PROGRAM RECOGNITION/MUTUAL } \\
\text { RECOGNITION ARRANGEMENTS: }\end{array}$ & No. Our list of chemicals is unique to our state sites \\
\hline
\end{tabular}




\begin{tabular}{|c|c|}
\hline DEPARTMENT/AGENCY : & $\begin{array}{l}\text { MINNESOTA DEPARTMENT OF AGRICULTURE, } \\
\text { LABORATORY SERVICES DIVISION }\end{array}$ \\
\hline ADDRESS : & $\begin{array}{l}90 \text { West Plato Blvd. } \\
\text { St. Paul, MN 55107-2094 }\end{array}$ \\
\hline & PHONE: 651-297-1901 \\
\hline & FAX: $\quad 651-297-8787$ \\
\hline NAME OF PROGRAM: & Interstate Milk Shippers Under Pasteurized Milk Ordinance \\
\hline PROGRAM DESCRIPTION: & $\begin{array}{l}\text { Certification program of on-site audits and split sampling, which } \\
\text { allows the interstate shipment of milk for Grade A fluid milk. }\end{array}$ \\
\hline DATE PROGRAM STARTED: & October 30,1965 . \\
\hline FIELDS OF TESTING: & \begin{tabular}{|ll}
$\square$ Acoustic and Vibration Testing & $\square$ Mechanical Testing \\
$\bigotimes$ Biological Testing & $\square$ Metrology \\
$\square$ Chemical Testing & $\square$ Nondestructive Testing \\
$\square$ Electrical Testing & $\square$ Optics and Photometry \\
$\square$ Ionizing Radiation & $\square$ Thermal Testing \\
$\square$ Other &
\end{tabular} \\
\hline $\begin{array}{l}\text { CATEGORIES OF PRODUCTS } \\
\text { COVERED BY THE PROGRAM: }\end{array}$ & Milk and milk products. \\
\hline $\begin{array}{l}\text { NUMBER OF LABS ACCREDITED/ } \\
\text { APPROVED : }\end{array}$ & 48 \\
\hline $\begin{array}{l}\text { ACCREDITATION/APPROVAL } \\
\text { VALID FOR: }\end{array}$ & 2 years. \\
\hline NUMBER OF ASSESSORS: & 1 . \\
\hline $\begin{array}{l}\text { MANDATORY ASSESSOR } \\
\text { QUALIFICATIONS : }\end{array}$ & \begin{tabular}{|c|c|}
$\square$ No & $\begin{array}{c}\text { Yes, training is required every three years } \\
\text { with on-site audits. }\end{array}$
\end{tabular} \\
\hline PARTICIPATION FEE: & 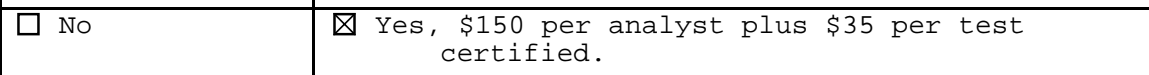 \\
\hline FEE SCHEDULE AVAILABLE: & 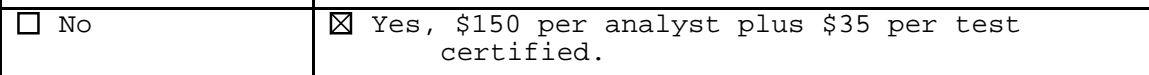 \\
\hline PROGRAM HAS AN APPEALS PROCEDURE : & $\begin{array}{l}\text { \ Yes, Appeals can be made to the Laboratory } \\
\text { Quality Assurance Branch of the FDA, } \\
\text { Chicago, IL. }\end{array}$ \\
\hline PROGRAM HAS A LOGO: & $\square$ Yes \\
\hline $\begin{array}{l}\text { PROGRAM IS OPEN TO } \\
\text { LABORATORIES: }\end{array}$ & $\begin{array}{ll}\text { All U.S. Private Sector } & \text { All U.S. Government } \\
\square \text { All Foreign Private Sector } & \square \text { All Foreign Government } \\
\square \text { Other } & \end{array}$ \\
\hline $\begin{array}{l}\text { AVAILABILITY OF PUBLISHED LIST OF } \\
\text { DIRECTORIES AVAILABLE: }\end{array}$ & \begin{tabular}{|l|l|}
$\square$ No & Yes, the list is distributed by the U.S. Food \\
& and Drug Administration, 200 'C' Street, \\
SW, Washington, DC.20204.
\end{tabular} \\
\hline $\begin{array}{l}\text { AVAILABILITY OF ASSESSMENT } \\
\text { CRITERIA/PROCEDURES: }\end{array}$ & $\begin{array}{l}\text { These are available from the U.S. Food and Drug Administration, } 200 \\
\text { 'C' Street, S.W., Washington, D.C. } 20204 \text {. }\end{array}$ \\
\hline
\end{tabular}




\begin{tabular}{|c|c|}
\hline $\begin{array}{l}\text { APPROVED/ACCREDITED LABORATORY } \\
\text { MANDATED REQUIREMENTS: }\end{array}$ & 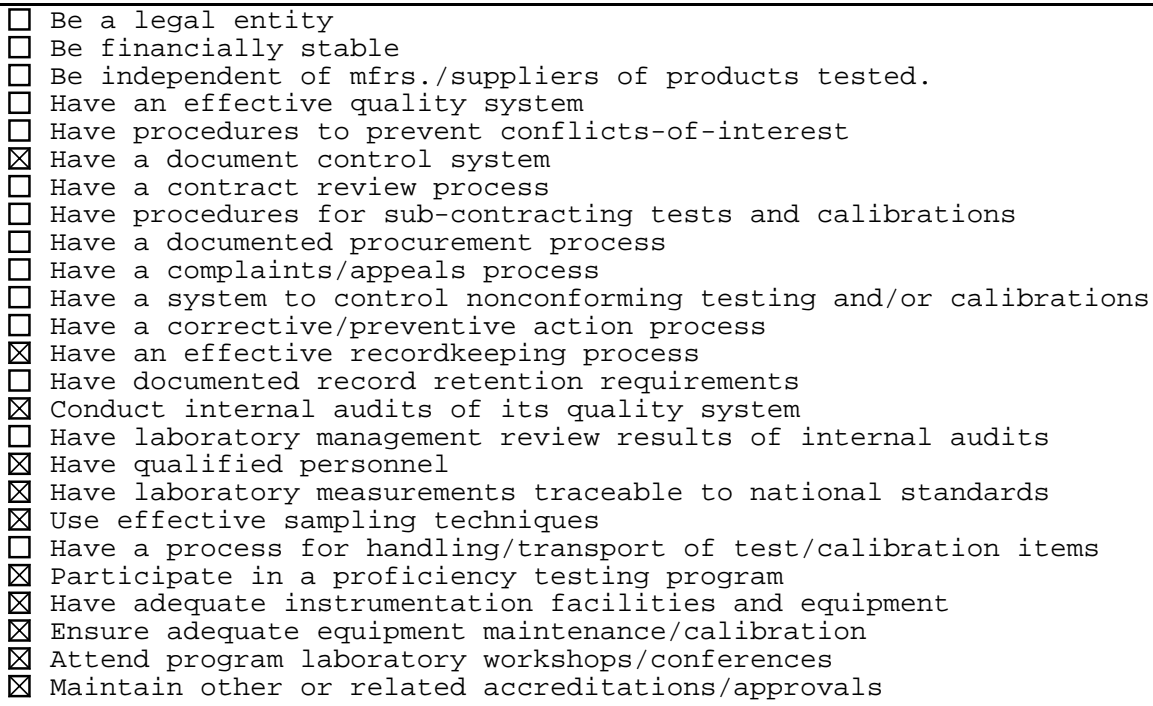 \\
\hline $\begin{array}{l}\text { STANDARD (S) WITH WHICH PROGRAM } \\
\text { CONFORMS : }\end{array}$ & $\begin{array}{r}\text { Yes, Complies with Food and Drug } \\
\text { Administration (FDA) standards } \\
\text { under Pasteurized Milk Ordinance. }\end{array}$ \\
\hline $\begin{array}{l}\text { PROGRAM RECOGNITION/MUTUAL } \\
\text { RECOGNITION ARRANGEMENTS: }\end{array}$ & Not appli \\
\hline
\end{tabular}




\begin{tabular}{|c|c|}
\hline DEPARTMENT/AGENCY : & NEW HAMPSHIRE DEPARTMENT OF ENVIRONMENTAL SERVICES \\
\hline ADDRESS : & $\begin{array}{l}\text { Program Manager, NH Environmental Laboratory Accreditation Program, } 6 \\
\text { Hazen Drive, P.O. Box 95, Concord, NH 03302-0095 }\end{array}$ \\
\hline & $\begin{array}{l}\text { INTERNET } \\
\text { URL: wWw.des.state.nh.us/nhelap }\end{array}$ \\
\hline & E-MAIL: cdyeredes.state.nh.us \\
\hline NAME OF PROGRAM: & New Hampshire Environmental Laboratory Accreditation Program. \\
\hline PROGRAM DESCRIPTION: & Accreditation of environmental laboratories. \\
\hline DATE PROGRAM STARTED: & 1988. \\
\hline FIELDS OF TESTING: & $\begin{array}{ll}\square \text { Acoustic and Vibration Testing } & \square \text { Mechanical Testing } \\
\text { Biological Testing } & \square \text { Metrology } \\
\text { \ Chemical Testing } & \square \text { Nondestructive Testing } \\
\square \text { Electrical Testing } & \square \text { Optics and Photometry } \\
\text { Ionizing Radiation } & \square \text { Thermal Testing } \\
\text { D Other - Whole effluent toxicity. } & \end{array}$ \\
\hline $\begin{array}{l}\text { CATEGORIES OF PRODUCTS } \\
\text { COVERED BY THE PROGRAM: }\end{array}$ & Drinking Water, Wastewater. \\
\hline $\begin{array}{l}\text { NUMBER OF LABS ACCREDITED/ } \\
\text { APPROVED: }\end{array}$ & 107. \\
\hline $\begin{array}{l}\text { ACCREDITATION/APPROVAL } \\
\text { VALID FOR: }\end{array}$ & 1 year. \\
\hline NUMBER OF ASSESSORS: & 2 . \\
\hline $\begin{array}{l}\text { MANDATORY ASSESSOR } \\
\text { QUALIFICATIONS : }\end{array}$ & \begin{tabular}{|l|l}
$\square$ No & $\begin{array}{r}\text { Yes, must hold a Bachelor's degree in biology, } \\
\text { microbiology, chemistry, environmental }\end{array}$ \\
science, chemical engineering or other \\
degree related to environmental or lab \\
sciences, NELAC approved training (if \\
available), 4 audits w/experienced assessor.
\end{tabular} \\
\hline PARTICIPATION FEE : & $\begin{array}{l}\bigotimes \text { Yes, available upon request by calling (603) } \\
\text { 271-2991. }\end{array}$ \\
\hline FEE SCHEDULE AVAILABLE: & $\begin{array}{l}\text { \ Yes, available upon request by calling (603) 271- } \\
\text { 2991. }\end{array}$ \\
\hline PROGRAM HAS AN APPEALS PROCEDURE: & 区 Yes, New Hampshire Water Council. \\
\hline PROGRAM HAS A LOGO: & $\triangle$ Yes, NELAP logo. \\
\hline $\begin{array}{l}\text { PROGRAM IS OPEN TO } \\
\text { LABORATORIES : }\end{array}$ & $\begin{array}{lll}\text { All U.S. Private Sector } & \bigotimes \text { All U.S. Government } \\
\square \text { All Foreign Private Sector } & \square \text { All Foreign Government } \\
\text { Other - NH Municipal labs. } & \end{array}$ \\
\hline $\begin{array}{l}\text { AVAILABILITY OF PUBLISHED LIST OF } \\
\text { LABORATORIES: }\end{array}$ & 囚 Yes, available on website. \\
\hline $\begin{array}{l}\text { AVAILABILITY OF ASSESSMENT } \\
\text { CRITERIA/PROCEDURES: }\end{array}$ & NELAC standards. \\
\hline
\end{tabular}




\begin{tabular}{|c|c|}
\hline $\begin{array}{l}\text { APPROVED/ACCREDITED LABORATORY } \\
\text { MANDATED REQUIREMENTS: }\end{array}$ & 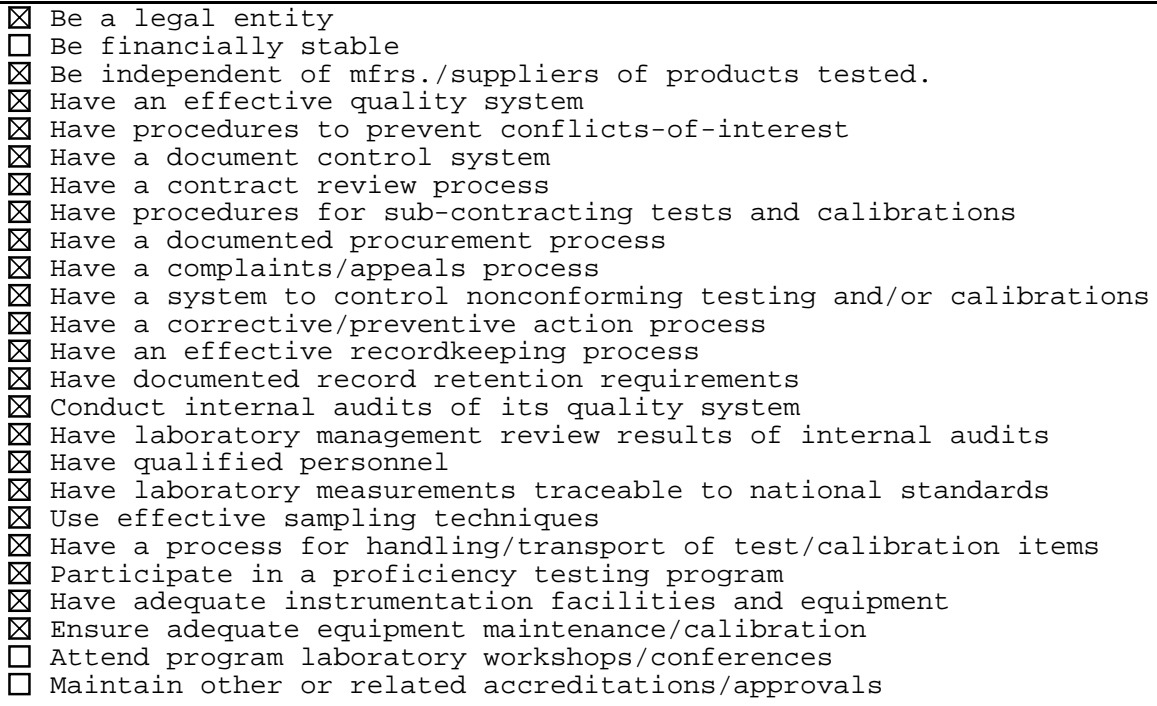 \\
\hline $\begin{array}{l}\text { STANDARD (S) WITH WHICH PROGRAM } \\
\text { CONFORMS: }\end{array}$ & 区 Yes, 1999 NELAC Standards. \\
\hline $\begin{array}{l}\text { PROGRAM RECOGNITION/MUTUAL } \\
\text { RECOGNITION ARRANGEMENTS : }\end{array}$ & diting Authorities. \\
\hline
\end{tabular}




\begin{tabular}{|c|c|}
\hline DEPARTMENT/AGENCY : & NEW JERSEY DEPARTMENT OF ENVIRONMENTAL PROTECTION \\
\hline ADDRESS : & $\begin{array}{l}\text { Chief, Office of Quality Assurance, P.O.Box 424, } 9 \text { Ewing Street, } \\
\text { Trenton, NJ } 08625\end{array}$ \\
\hline & INTERNET URL: $\mathrm{N} / \mathrm{A}$ \\
\hline & E-MAIL: jaiello@dep.state.nj.us \\
\hline NAME OF PROGRAM: & New Jersey Environmental Laboratory Certification Program \\
\hline PROGRAM DESCRIPTION: & $\begin{array}{l}\text { Administer activities to ensure that NJ's environmental community has } \\
\text { accurate \& reliable analytical data to make management, regulatory } \\
\text { and scientific decisions. Also administer regulations \& policies to } \\
\text { assure the quality of environmental laboratory data used by the } \\
\text { NJDEP, industry, local government, municipal authorities \& private } \\
\text { citizens. Certifies and licenses the analytical capabilities of } \\
\text { environmental laboratories that analyze NJ drinking water, } \\
\text { groundwater, wastewater, ambient water, air, soil, solid waste, } \\
\text { hazardous waste and sludge for microbiologicl, inorganic, organic, } \\
\text { radiochemical, radon \& biological properties. }\end{array}$ \\
\hline DATE PROGRAM STARTED: & 1979. \\
\hline FIELDS OF TESTING: & $\begin{array}{ll}\square \text { Acoustic and Vibration Testing } & \square \text { Mechanical Testing } \\
\bigotimes \text { Biological Testing } & \square \text { Metrology } \\
\bigotimes \text { Chemical Testing } & \square \text { Nondestructive Testing } \\
\square \text { Electrical Testing } & \square \text { Optics and Photometry } \\
\text { Ionizing Radiation } & \square \text { Thermal Testing } \\
\square \text { Other } & \end{array}$ \\
\hline $\begin{array}{l}\text { CATEGORIES OF PRODUCTS } \\
\text { COVERED BY THE PROGRAM: }\end{array}$ & Environmental matrices. \\
\hline $\begin{array}{l}\text { NUMBER OF LABS ACCREDITED/ } \\
\text { APPROVED: }\end{array}$ & 800 \\
\hline $\begin{array}{l}\text { ACCREDITATION/APPROVAL } \\
\text { VALID FOR: }\end{array}$ & Up to one year. \\
\hline NUMBER OF ASSESSORS: & 17 . \\
\hline $\begin{array}{l}\text { MANDATORY ASSESSOR } \\
\text { QUALIFICATIONS : }\end{array}$ & $\begin{array}{l}\text { \ Yes, must have appropriate college degrees and } \\
\text { lab experience. }\end{array}$ \\
\hline PARTICIPATION FEE: & $\begin{array}{l}\bigotimes \text { Yes, available from NJ Dept. of Environmental } \\
\text { Protection, Office of Quality Assurance, at } \\
\text { the address listed above. }\end{array}$ \\
\hline FEE SCHEDULE AVAILABLE: & $\begin{array}{l}\text { Yes, available from NJ Dept.Environmental } \\
\text { Protection, Office of Quality Assurance, at } \\
\text { the address listed above. }\end{array}$ \\
\hline PROGRAM HAS AN APPEALS PROCEDURE : & $\begin{array}{c}\triangle \text { Yes, NJ Dept.of Environmental Protection and NJ } \\
\text { Attorney General's Office. }\end{array}$ \\
\hline PROGRAM HAS A LOGO: & $\square$ Yes \\
\hline $\begin{array}{l}\text { PROGRAM IS OPEN TO } \\
\text { LABORATORIES : }\end{array}$ & $\begin{array}{l}\square \text { All U.S. Private Sector } \\
\square \text { All Foreign Private Sector } \\
\square \text { other }\end{array}$ \\
\hline $\begin{array}{l}\text { AVAILABILITY OF PUBLISHED LIST OF } \\
\text { LABORATORIES: }\end{array}$ & $\begin{array}{l}\text { \Yes, available upon request from NJ DEP, Office } \\
\text { of Quality Assurance, at the address } \\
\text { above. }\end{array}$ \\
\hline $\begin{array}{l}\text { AVAILABILITY OF ASSESSMENT } \\
\text { CRITERIA/PROCEDURES : }\end{array}$ & $\begin{array}{l}\text { Available upon request from NJ DEP, Office of Quality Assurance, at } \\
\text { the address listed above. }\end{array}$ \\
\hline
\end{tabular}




\begin{tabular}{|c|c|}
\hline $\begin{array}{l}\text { APPROVED/ACCREDITED LABORATORY } \\
\text { MANDATED REQUIREMENTS: }\end{array}$ & 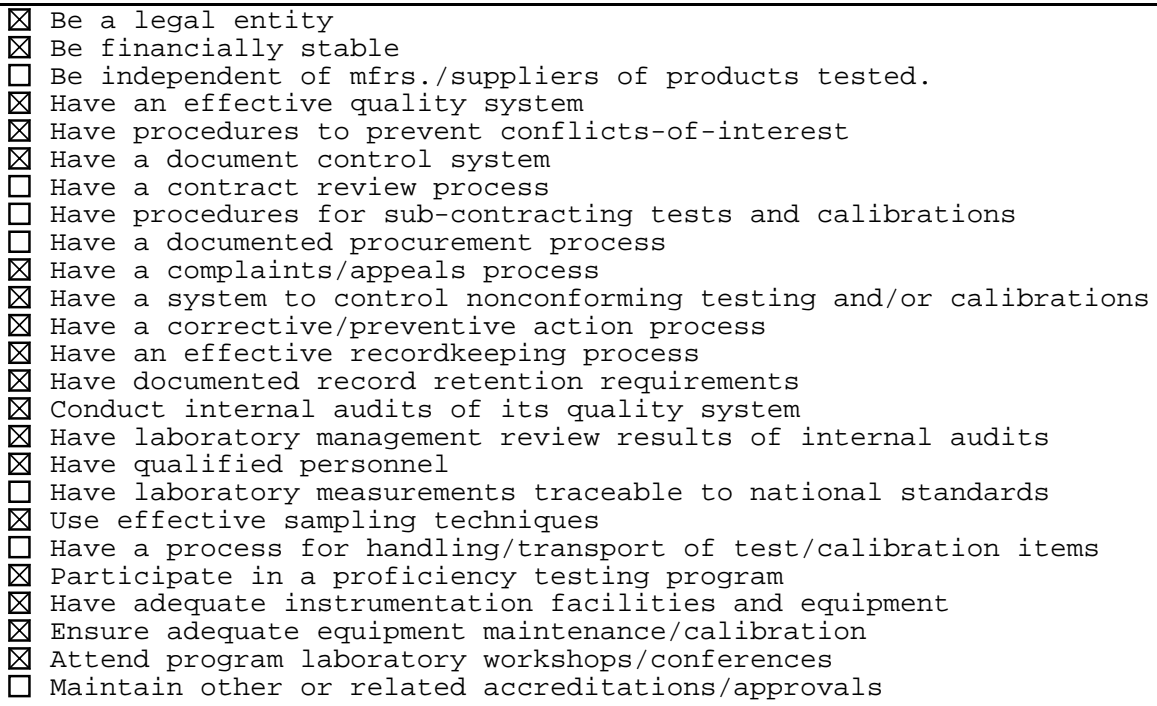 \\
\hline $\begin{array}{l}\text { STANDARD (S) WITH WHICH PROGRAM } \\
\text { CONFORMS : }\end{array}$ & $\begin{array}{l}\text { Yes, those of the National } \\
\text { Environmental Laboratory } \\
\text { Accreditation Program \& others. }\end{array}$ \\
\hline $\begin{array}{l}\text { PROGRAM RECOGNITION/MUTUAL } \\
\text { RECOGNITION ARRANGEMENTS : }\end{array}$ & hal Environmental Laboratory Accreditation Conference. \\
\hline
\end{tabular}




\begin{tabular}{|c|c|}
\hline DEPARTMENT/AGENCY : & $\begin{array}{l}\text { NEW MEXICO ENVIRONMENTAL DEPARTMENT-DRINKING WATER } \\
\text { BUREAU }\end{array}$ \\
\hline ADDRESS : & $\begin{array}{l}\text { Program Manager, Drinking Water Bureau, } 525 \text { Camino De Los Marquez, } \\
\text { suite 4, Santa Fe, NM } 87501\end{array}$ \\
\hline & INTERNET URL: wWw.nmenv.state.nm.us \\
\hline & $\begin{array}{l}\text { E-MAIL: } \\
\text { stephen.wust@nmenv. state.nm.us }\end{array}$ \\
\hline NAME OF PROGRAM: & Drinking Water \\
\hline PROGRAM DESCRIPTION: & Primary agency for Safe Drinking water Act regulations. \\
\hline DATE PROGRAM STARTED: & Not available. \\
\hline FIELDS OF TESTING: & $\begin{array}{ll}\text { Acoustic and Vibration Testing } & \square \text { Mechanical Testing } \\
\square \text { Biological Testing } & \square \text { Metrology } \\
\square \text { Chemical Testing } & \square \text { Nondestructive Testing } \\
\square \text { Electrical Testing } & \square \text { Optics and Photometry } \\
\square \text { Ionizing Radiation } & \square \text { Thermal Testing } \\
\text { D Other Regulatory certification of drinking water compliance } \\
\text { analysis-no accreditation. }\end{array}$ \\
\hline $\begin{array}{l}\text { CATEGORIES OF PRODUCTS } \\
\text { COVERED BY THE PROGRAM: }\end{array}$ & Drinking water. \\
\hline $\begin{array}{l}\text { NUMBER OF LABS ACCREDITED/ } \\
\text { APPROVED: }\end{array}$ & 27 for microbiology, 3 for chemistry. \\
\hline $\begin{array}{l}\text { ACCREDITATION/APPROVAL } \\
\text { VALID FOR: }\end{array}$ & 2 and 4 years. \\
\hline NUMBER OF ASSESSORS: & 1 \\
\hline $\begin{array}{l}\text { MANDATORY ASSESSOR } \\
\text { QUALIFICATIONS : }\end{array}$ & $\square$ Yes \\
\hline PARTICIPATION FEE : & $\square$ Yes \\
\hline FEE SCHEDULE AVAILABLE: & $\square$ Yes \\
\hline PROGRAM HAS AN APPEALS PROCEDURE: & $\square$ Yes \\
\hline PROGRAM HAS A LOGO: & Q Yes, available upon request. \\
\hline $\begin{array}{l}\text { PROGRAM IS OPEN TO } \\
\text { LABORATORIES : }\end{array}$ & $\begin{array}{ll}\text { All U.S. Private Sector } & \text { All U.S. Government } \\
\bigotimes \text { All Foreign Private Sector } & \text { All Foreign Government } \\
\square \text { Other } & \end{array}$ \\
\hline $\begin{array}{l}\text { AVAILABILITY OF PUBLISHED LIST OF } \\
\text { LABORATORIES: }\end{array}$ & $\square$ Yes \\
\hline $\begin{array}{l}\text { AVAILABILITY OF ASSESSMENT } \\
\text { CRITERIA/PROCEDURES : }\end{array}$ & $\begin{array}{l}\text { Available on request. Only Mandated Requirement is that the } \\
\text { laboratory be accredited. }\end{array}$ \\
\hline
\end{tabular}




\begin{tabular}{|c|c|}
\hline $\begin{array}{l}\text { APPROVED/ACCREDITED LABORATORY } \\
\text { MANDATED REQUIREMENTS: }\end{array}$ & $\begin{array}{l}\square \text { Be a legal entity } \\
\square \text { Be financially stable } \\
\square \text { Be independent of mfrs./suppliers of products tested. } \\
\square \text { Have an effective quality system } \\
\square \text { Have procedures to prevent conflicts-of-interest } \\
\square \text { Have a document control system } \\
\square \text { Have a contract review process } \\
\square \text { Have procedures for sub-contracting tests and calibrations } \\
\square \text { Have a documented procurement process } \\
\square \text { Have a complaints/appeals process } \\
\square \text { Have a system to control nonconforming testing and/or calibrations } \\
\square \text { Have a corrective/preventive action process } \\
\square \text { Have an effective recordkeeping process } \\
\square \text { Have documented record retention requirements } \\
\square \text { Conduct internal audits of its quality system } \\
\square \text { Have laboratory management review results of internal audits } \\
\square \text { Have qualified personnel } \\
\square \text { Have laboratory measurements traceable to national standards } \\
\square \text { Use effective sampling techniques } \\
\square \text { Have a process for handling/transport of test/calibration items } \\
\square \text { Participate in a proficiency testing program } \\
\square \text { Have adequate instrumentation facilities and equipment } \\
\square \text { Ensure adequate equipment maintenance/calibration } \\
\square \text { Attend program laboratory workshops/conferences } \\
\square \text { Maintain other or related accreditations/approvals }\end{array}$ \\
\hline $\begin{array}{l}\text { STANDARD (S) WITH WHICH PROGRAM } \\
\text { CONEORMS : }\end{array}$ & 区 No $\square$ Don't know \\
\hline $\begin{array}{l}\text { PROGRAM RECOGNITION/MUTUAL } \\
\text { RECOGNITION ARRANGEMENTS : }\end{array}$ & None. \\
\hline
\end{tabular}




\begin{tabular}{|c|c|}
\hline DEPARTMENT/AGENCY : & $\begin{array}{l}\text { NEW YORK STATE DEPARTMENT OF AGRICULTURE AND MARKETS, } \\
\text { DIVISION OF MILK CONTROL }\end{array}$ \\
\hline ADDRESS : & II Winners Circle, Albany, New York 12235. \\
\hline & PHONE : $518-457-8873$ \\
\hline & E-MAIL: jim.fitts@agmkt.state.ny.us \\
\hline NAME OF PROGRAM: & $\begin{array}{l}\text { National Conference on Interstate Milk Shipments (NCIMS) Laboratory } \\
\text { Accreditation Program. }\end{array}$ \\
\hline PROGRAM DESCRIPTION: & $\begin{array}{l}\text { NCIMS is a voluntary cooperative program between the states and FDA, } \\
\text { to achieve uniformity in regulations governing the dairy industry and } \\
\text { interstate trade of dairy products. }\end{array}$ \\
\hline DATE PROGRAM STARTED: & 1987 . \\
\hline FIELDS OF TESTING: & $\begin{array}{lll}\square \text { Acoustic and Vibration Testing } & \square \text { Mechanical Testing } \\
\square \text { Biological Testing } & \square \text { Metrology } \\
\square \text { Chemical Testing } & \square \text { Nondestructive Testing } \\
\square \text { Electrical Testing } & \square \text { Optics and Photometry } \\
\square \text { Ionizing Radiation } & \square \text { Thermal Testing } \\
\bigotimes \text { Other Milk testing. } & \end{array}$ \\
\hline $\begin{array}{l}\text { CATEGORIES OF PRODUCTS } \\
\text { COVERED BY THE PROGRAM: }\end{array}$ & Milk. \\
\hline $\begin{array}{l}\text { NUMBER OF LABS ACCREDITED/ } \\
\text { APPROVED : }\end{array}$ & 100 \\
\hline $\begin{array}{l}\text { ACCREDITATION/APPROVAL } \\
\text { VALID FOR: }\end{array}$ & 2 years. \\
\hline NUMBER OF ASSESSORS: & 2 . \\
\hline $\begin{array}{l}\text { MANDATORY ASSESSOR } \\
\text { QUALIFICATIONS : }\end{array}$ & \begin{tabular}{|c|c|}
$\square$ No & Yes, certification is conducted by Food and \\
Drug Administration (FDA), Laboratory \\
Quality Assurance Branch (LQAB).
\end{tabular} \\
\hline PARTICIPATION FEE: & $\square$ Yes \\
\hline FEE SCHEDULE AVAILABLE: & $\square$ Yes \\
\hline PROGRAM HAS AN APPEALS PROCEDURE: & $\square$ Yes \\
\hline PROGRAM HAS A LOGO: & $\square$ Yes \\
\hline $\begin{array}{l}\text { PROGRAM IS OPEN TO } \\
\text { LABORATORIES: }\end{array}$ & $\begin{array}{|ll|}\text { All U.S. Private Sector } & \text { All U.S. Government } \\
\square \text { All Foreign Private Sector } & \square \text { All Foreign Government } \\
\square \text { Other } & \end{array}$ \\
\hline $\begin{array}{l}\text { AVAILABILITY OF PUBLISHED LIST OF } \\
\text { DIRECTORIES AVAILABLE: }\end{array}$ & \begin{tabular}{l|l} 
Yes, they are published by FDA: It is the IMS \\
List of Sanitation Compliance and \\
Enforcement Ratings of Interstate Milk \\
Shippers. Also published on the internet. \\
The URL is available on request.
\end{tabular} \\
\hline $\begin{array}{l}\text { AVAILABILITY OF ASSESSMENT } \\
\text { CRITERIA/PROCEDURES: }\end{array}$ & $\begin{array}{l}\text { "Evaluation of Milk Laboratories" available from the U.S. Department } \\
\text { of Health and Human Services (HHS), Public Health Services (PHS), and } \\
\text { Food and Drug Administration (FDA). }\end{array}$ \\
\hline
\end{tabular}




\begin{tabular}{|c|c|}
\hline $\begin{array}{l}\text { APPROVED/ACCREDITED LABORATORY } \\
\text { MANDATED REQUIREMENTS: }\end{array}$ & 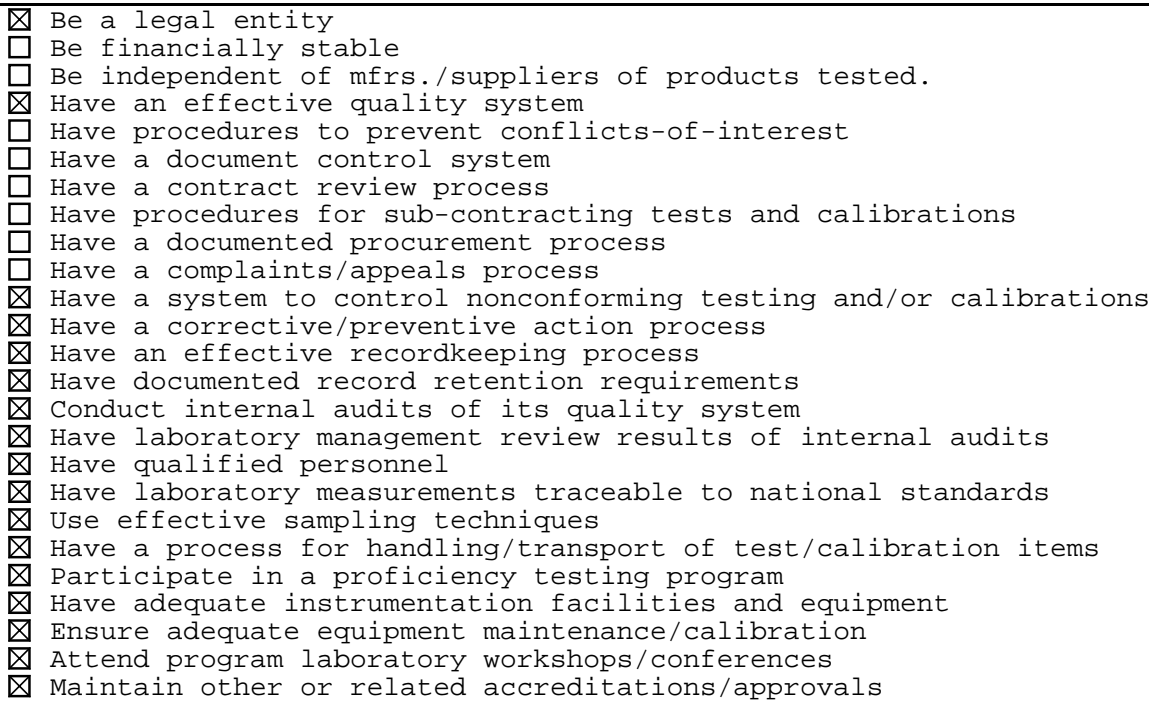 \\
\hline $\begin{array}{l}\text { STANDARD (S) WITH WHICH PROGRAM } \\
\text { CONFORMS : }\end{array}$ & 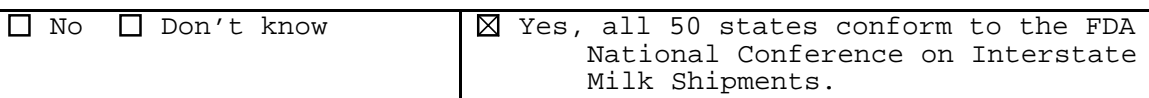 \\
\hline $\begin{array}{l}\text { PROGRAM RECOGNITION/MUTUAL } \\
\text { RECOGNITION ARRANGEMENTS: }\end{array}$ & $\begin{array}{l}\text { Program is recognized throughout the U.S. The FDA/NCIMS program is a } \\
\text { cooperative program between the states and FDA to ensure uniformity } \\
\text { between the states in the regulation of the dairy industry. }\end{array}$ \\
\hline
\end{tabular}




\begin{tabular}{|c|c|}
\hline DEPARTMENT/AGENCY : & $\begin{array}{l}\text { NEW YORK-THE CITY OF NEW YORK-DEPARTMENT OF BUILDINGS- } \\
\text { MATERIALS AND EQUIPMENT ACCEPTANCE DIVISION [MEA] }\end{array}$ \\
\hline \multirow[t]{3}{*}{ ADDRESS : } & $\begin{array}{l}\text { Department of Buildings, 14th Floor, } 60 \text { Hudson Street, New York, NY } \\
10013-3394\end{array}$ \\
\hline & INTERNET URL: wWW.nyc.gov/buildings \\
\hline & E-MAIL: info@nyc.gov/buildings \\
\hline NAME OF PROGRAM: & $\begin{array}{l}\text { MEA Acceptable Testing Service or Laboratory and Approved Concrete } \\
\text { Testing Laboratories }\end{array}$ \\
\hline PROGRAM DESCRIPTION: & $\begin{array}{l}\text { Determination of acceptability of testing and inspection laboratories } \\
\text { and firms to provide data on compliance of products on installation } \\
\text { meeting New York City Building Codes for mechanical requirements. }\end{array}$ \\
\hline DATE PROGRAM STARTED: & 1965 revisions. \\
\hline FIELDS OF TESTING: & $\begin{array}{ll}\text { Acoustic and Vibration Testing } & \text { Mechanical Testing } \\
\text { Biological Testing } & \square \text { Metrology } \\
\text { \ Chemical Testing } & \square \text { Nondestructive Testing } \\
\text { Electrical Testing } & \square \text { Optics and Photometry } \\
\square \text { Ionizing Radiation } & \square \text { Thermal Testing } \\
\square \text { Other } & \end{array}$ \\
\hline $\begin{array}{l}\text { CATEGORIES OF PRODUCTS } \\
\text { COVERED BY THE PROGRAM: }\end{array}$ & $\begin{array}{l}\text { Construction and building products such as air conditioning, } \\
\text { hardware, finishes and flooring. }\end{array}$ \\
\hline $\begin{array}{l}\text { NUMBER OF LABS ACCREDITED/ } \\
\text { APPROVED: }\end{array}$ & 110 as of April 2001. \\
\hline $\begin{array}{l}\text { ACCREDITATION/APPROVAL } \\
\text { VALID FOR: }\end{array}$ & Until revoked. \\
\hline NUMBER OF ASSESSORS: & 3. \\
\hline $\begin{array}{l}\text { MANDATORY ASSESSOR } \\
\text { QUALIFICATIONS : }\end{array}$ & \begin{tabular}{|c|c}
$\square$ No & $\begin{array}{c}\text { Yes, a mechanical engineering degree is } \\
\text { required. }\end{array}$
\end{tabular} \\
\hline PARTICIPATION FEE: & \begin{tabular}{|l|l|} 
No & $\square$ Yes \\
\end{tabular} \\
\hline FEE SCHEDULE AVAILABLE: & $\square$ Yes \\
\hline PROGRAM HAS AN APPEALS PROCEDURE: & $\begin{array}{l}\bigotimes \text { Yes, normal New York City due process appeals } \\
\text { system, as well as appeals to the Building } \\
\text { Commissioner appeal. }\end{array}$ \\
\hline PROGRAM HAS A LOGO: & \ $\mid$ Yes, issued acceptance number. \\
\hline $\begin{array}{l}\text { PROGRAM IS OPEN TO } \\
\text { LABORATORIES : }\end{array}$ & $\begin{array}{ll}\text { All U.S. Private Sector } & \text { All U.S. Government } \\
\square \text { All Foreign Private Sector } & \square \text { All Foreign Government } \\
\square \text { Other } & \end{array}$ \\
\hline $\begin{array}{l}\text { AVAILABILITY OF PUBLISHED LIST OF } \\
\text { LABORATORIES: }\end{array}$ & 凶 Yes, available upon request. \\
\hline $\begin{array}{l}\text { AVAILABILITY OF ASSESSMENT } \\
\text { CRITERIA/PROCEDURES : }\end{array}$ & Available upon request. \\
\hline
\end{tabular}




\begin{tabular}{|c|c|}
\hline $\begin{array}{l}\text { APPROVED/ACCREDITED LABORATORY } \\
\text { MANDATED REQUIREMENTS: }\end{array}$ & $\begin{array}{l}\text { Be legal entity } \\
\square \text { Be financially stable } \\
\bigotimes \text { Be independent of mfrs./suppliers of products tested. } \\
\text { Have an effective quality system } \\
\text { Have procedures to prevent conflicts-of-interest } \\
\square \text { Have a document control system } \\
\text { Have a contract review process } \\
\text { Have procedures for sub-contracting tests and calibrations } \\
\text { Have a documented procurement process } \\
\square \text { Have a complaints/appeals process } \\
\text { Have a system to control nonconforming testing and/or calibrations } \\
\text { Have a corrective/preventive action process } \\
\text { Have an effective recordkeeping process } \\
\text { Have documented record retention requirements } \\
\text { Honduct internal audits of its quality system } \\
\text { Have laboratory management review results of internal audits } \\
\text { Have qualified personnel } \\
\text { Have laboratory measurements traceable to national standards } \\
\square \text { Use effective sampling techniques } \\
\square \text { Have a process for handling/transport of test/calibration items } \\
\square \text { Participate in a proficiency testing program } \\
\text { Have adequate instrumentation facilities and equipment } \\
\square \text { Ensure adequate equipment maintenance/calibration } \\
\square \text { Attend program laboratory workshops/conferences } \\
\square \text { Maintain other or related accreditations/approvals }\end{array}$ \\
\hline $\begin{array}{l}\text { STANDARD (S) WITH WHICH PROGRAM } \\
\text { CONFORMS: }\end{array}$ & $\square$ No $\quad$ Don't know \\
\hline $\begin{array}{l}\text { PROGRAM RECOGNITION/MUTUAL } \\
\text { RECOGNITION ARRANGEMENTS : }\end{array}$ & None. \\
\hline
\end{tabular}




\begin{tabular}{|c|c|}
\hline DEPARTMENT/AGENCY : & $\begin{array}{l}\text { NORTH CAROLINA DEPARTMENT OF INSURANCE, OFFICE OF STATE } \\
\text { FIRE MARSHAL AND N.C. BUILDING CODE COUNCIL }\end{array}$ \\
\hline \multirow[t]{3}{*}{ ADDRESS : } & State Electrical Inspector, 410 N. Boylan Ave., Raleigh, NC 27603 \\
\hline & \begin{tabular}{l|l} 
PHONE : 919-733-3901 & INTERNET URL: N/A
\end{tabular} \\
\hline & FAX: $919-715-4607$ \\
\hline NAME OF PROGRAM: & $\begin{array}{l}\text { State of North Carolina: Acceptance of laboratories to provide } \\
\text { evidence of safety of electrical and mechanical goods. }\end{array}$ \\
\hline PROGRAM DESCRIPTION: & $\begin{array}{l}\text { Acceptance of electrical and mechanical products by laboratories } \\
\text { accredited by the N.C. Building Code Council [NCBCC] that can } \\
\text { demonstrate capability to test and examine electrical and mechanical } \\
\text { products and modular structures that are required to be approved in } \\
\text { accordance with the North Carolina Building code. }\end{array}$ \\
\hline DATE PROGRAM STARTED: & 1982. \\
\hline FIELDS OF TESTING: & $\begin{array}{ll}\square \text { Acoustic and Vibration Testing } & \text { Mechanical Testing } \\
\square \text { Biological Testing } & \square \text { Metrology } \\
\square \text { Chemical Testing } & \square \text { Nondestructive Testing } \\
\bigotimes \text { Electrical Testing } & \square \text { Optics and Photometry } \\
\square \text { Ionizing Radiation } & \square \text { Thermal Testing } \\
\square \text { Other } & \end{array}$ \\
\hline $\begin{array}{l}\text { CATEGORIES OF PRODUCTS } \\
\text { COVERED BY THE PROGRAM: }\end{array}$ & $\begin{array}{l}53 \text { equipment categories listed, which is available upon request and } \\
\text { which covers building and construction materials ranging from } \\
\text { conductors for general wiring through insulating tape and closures. }\end{array}$ \\
\hline $\begin{array}{l}\text { NUMBER OF LABS ACCREDITED/ } \\
\text { APPROVED : }\end{array}$ & 12 . \\
\hline $\begin{array}{l}\text { ACCREDITATION/APPROVAL } \\
\text { VALID FOR: }\end{array}$ & 1 year. \\
\hline NUMBER OF ASSESSORS: & Not available. \\
\hline $\begin{array}{l}\text { MANDATORY ASSESSOR } \\
\text { QUALIFICATIONS : }\end{array}$ & \begin{tabular}{l|l} 
No & $\square$ Yes
\end{tabular} \\
\hline PARTICIPATION FEE : & \Yes \\
\hline FEE SCHEDULE AVAILABLE: & \ Yes, available upon request. \\
\hline PROGRAM HAS AN APPEALS PROCEDURE : & 区 Yes, North Carolina Building Code Council \\
\hline PROGRAM HAS A LOGO: & $\square$ Yes \\
\hline $\begin{array}{l}\text { PROGRAM IS OPEN TO } \\
\text { LABORATORIES: }\end{array}$ & $\begin{array}{ll}\text { 冈 All U.S. Private Sector } & \text { All U.S. Government } \\
\bigotimes \text { All Foreign Private Sector } & \text { All Foreign Government } \\
\square \text { Other } & \end{array}$ \\
\hline $\begin{array}{l}\text { AVAILABILITY OF PUBLISHED LIST OF } \\
\text { LABORATORIES: }\end{array}$ & 凶Yes, available upon request. \\
\hline $\begin{array}{l}\text { AVAILABILITY OF ASSESSMENT } \\
\text { CRITERIA/PROCEDURES: }\end{array}$ & Available upon request. \\
\hline
\end{tabular}




\begin{tabular}{|c|c|}
\hline $\begin{array}{l}\text { APPROVED/ACCREDITED LABORATORY } \\
\text { MANDATED REQUIREMENTS: }\end{array}$ & $\begin{array}{l}\text { Be a legal entity } \\
\text { Be financially stable } \\
\text { Be independent of mfrs./suppliers of products tested. } \\
\text { Have an effective quality system } \\
\text { Have procedures to prevent conflicts-of-interest } \\
\text { Have a document control system } \\
\text { Have a contract review process } \\
\text { Have procedures for sub-contracting tests and calibrations } \\
\text { Have a documented procurement process } \\
\text { Have a complaints/appeals process } \\
\text { Have a system to control nonconforming testing and/or calibrations } \\
\text { Have a corrective/preventive action process } \\
\text { Have an effective recordkeeping process } \\
\text { Have documented record retention requirements } \\
\text { H Conduct internal audits of its quality system } \\
\text { Have laboratory management review results of internal audits } \\
\text { Have qualified personnel } \\
\text { Have laboratory measurements traceable to national standards } \\
\text { Hse effective sampling techniques } \\
\text { Unave a process for handling/transport of test/calibration items } \\
\square \text { Participate in a proficiency testing program } \\
\text { Have adequate instrumentation facilities and equipment } \\
\text { Hensure adequate equipment maintenance/calibration } \\
\square \text { Attend program laboratory workshops/conferences } \\
\square \text { Maintain other or related accreditations/approvals }\end{array}$ \\
\hline $\begin{array}{l}\text { STANDARD (S) WITH WHICH PROGRAM } \\
\text { CONFORMS : }\end{array}$ & $\square$ No $\otimes$ Don't know \\
\hline $\begin{array}{l}\text { PROGRAM RECOGNITION/MUTUAL } \\
\text { RECOGNITION ARRANGEMENTS : }\end{array}$ & Nor \\
\hline
\end{tabular}




\begin{tabular}{|c|c|}
\hline DEPARTMENT/AGENCY : & OHIO BOARD OF BUILDING STANDARDS \\
\hline \multirow[t]{3}{*}{ ADDRESS : } & P.O. Box 4009, 6606 Tussing Road, Reynoldsburg, Ohio 43068-9009 \\
\hline & \begin{tabular}{l|l} 
PHONE : 614-644-2613 & INTERNET URL: \\
& wWw.com.state.oh.us/odoc/dic
\end{tabular} \\
\hline & \begin{tabular}{l|l} 
FAX: & E-MAIL: wmbrady@com.state.oh.us
\end{tabular} \\
\hline NAME OF PROGRAM: & Approved Agencies. \\
\hline PROGRAM DESCRIPTION: & $\begin{array}{l}\text { To establish accreditation of testing/inspection agencies in } \\
\text { accordance with requirements of the Ohio State Basic Building Code } \\
\text { and other rules of the Board of Building Standards. }\end{array}$ \\
\hline DATE PROGRAM STARTED: & 1965. \\
\hline FIELDS OF TESTING: & $\begin{array}{ll}\text { Acoustic and Vibration Testing } & \text { Mechanical Testing } \\
\square \text { Biological Testing } & \square \text { Metrology } \\
\bigotimes \text { Chemical Testing } & \square \text { Nondestructive Testing } \\
\bigotimes \text { Electrical Testing } & \square \text { Optics and Photometry } \\
\square \text { Ionizing Radiation } & \square \text { Thermal Testing } \\
\square \text { Other } & \end{array}$ \\
\hline $\begin{array}{l}\text { CATEGORIES OF PRODUCTS } \\
\text { COVERED BY THE PROGRAM: }\end{array}$ & $\begin{array}{l}\text { Products, materials and assemblies are required by the Ohio Basic } \\
\text { Building Code to be approved, labeled or grade-marked for use in } \\
\text { building construction. }\end{array}$ \\
\hline $\begin{array}{l}\text { NUMBER OF LABS ACCREDITED/ } \\
\text { APPROVED: }\end{array}$ & 49. \\
\hline $\begin{array}{l}\text { ACCREDITATION/APPROVAL } \\
\text { VALID FOR: }\end{array}$ & Until expiration date listed on their certification. \\
\hline NUMBER OF ASSESSORS : & 7 . \\
\hline $\begin{array}{l}\text { MANDATORY ASSESSOR } \\
\text { QUALIFICATIONS : }\end{array}$ & \begin{tabular}{|l|l}
$\square$ No & Y Yes, Appendix P of Code.
\end{tabular} \\
\hline PARTICIPATION FEE : & $\square$ Yes \\
\hline FEE SCHEDULE AVAILABLE: & 区 Yes, Rule 4101:2-1-54 available upon request. \\
\hline PROGRAM HAS AN APPEALS PROCEDURE : & \ Yes, available upon request. \\
\hline PROGRAM HAS A LOGO: & $\square$ Yes \\
\hline $\begin{array}{l}\text { PROGRAM IS OPEN TO } \\
\text { LABORATORIES : }\end{array}$ & $\begin{array}{ll}\text { All U.S. Private Sector } & \text { All U.S. Government } \\
\text { All Foreign Private Sector } & \text { All Foreign Government } \\
\square \text { Other } & \end{array}$ \\
\hline $\begin{array}{l}\text { AVAILABILITY OF PUBLISHED LIST OF } \\
\text { LABORATORIES: }\end{array}$ & 囚 Yes, available upon request. \\
\hline $\begin{array}{l}\text { AVAILABILITY OF ASSESSMENT } \\
\text { CRITERIA/PROCEDURES: }\end{array}$ & Available upon request. \\
\hline
\end{tabular}




\begin{tabular}{|c|c|}
\hline $\begin{array}{l}\text { APPROVED/ACCREDITED LABORATORY } \\
\text { MANDATED REQUIREMENTS: }\end{array}$ & $\begin{array}{l}\text { Be a legal entity } \\
\square \text { Be financially stable } \\
\text { Be independent of mfrs./suppliers of products tested. } \\
\text { Have an effective quality system } \\
\text { Have procedures to prevent conflicts-of-interest } \\
\square \text { Have a document control system } \\
\square \text { Have a contract review process } \\
\text { Have procedures for sub-contracting tests and calibrations } \\
\square \text { Have a documented procurement process } \\
\square \text { Have a complaints/appeals process } \\
\bigotimes \text { Have a system to control nonconforming testing and/or calibrations } \\
\bigotimes \text { Have a corrective/preventive action process } \\
\bigotimes \text { Have an effective recordkeeping process } \\
\square \text { Have documented record retention requirements } \\
\text { Conduct internal audits of its quality system } \\
\bigotimes \text { Have laboratory management review results of internal audits } \\
\text { Have qualified personnel } \\
\text { Have laboratory measurements traceable to national standards } \\
\square \text { Use effective sampling techniques } \\
\square \text { Have a process for handling/transport of test/calibration items } \\
\square \text { Participate in a proficiency testing program } \\
\bigotimes \text { Have adequate instrumentation facilities and equipment } \\
\text { Ensure adequate equipment maintenance/calibration } \\
\square \text { Attend program laboratory workshops/conferences } \\
\square \text { Maintain other or related accreditations/approvals }\end{array}$ \\
\hline $\begin{array}{l}\text { STANDARD (S) WITH WHICH PROGRAM } \\
\text { CONEORMS: }\end{array}$ & $\square$ No D Don't know \\
\hline $\begin{array}{l}\text { PROGRAM RECOGNITION/MUTUAL } \\
\text { RECOGNITION ARRANGEMENTS : }\end{array}$ & None. \\
\hline
\end{tabular}




\begin{tabular}{|c|c|}
\hline DEPARTMENT/AGENCY : & $\begin{array}{l}\text { OHIO, CITY OF CLEVELAND, DEPARTMENT OF COMMUNITY } \\
\text { DEVELOPMENT, DIVISION OF BUILDING AND HOUSING }\end{array}$ \\
\hline \multirow[t]{3}{*}{ ADDRESS : } & 601 Lakeside Avenue, Room 320, Cleveland, Ohio 44114-1070. \\
\hline & PHONE : $216-664-2418$ \\
\hline & E-MAIL: N/A \\
\hline NAME OF PROGRAM: & Approved Testing Laboratory Listing. \\
\hline PROGRAM DESCRIPTION: & $\begin{array}{l}\text { To list approved testing laboratories for energy, electrical safety } \\
\text { and gas-fired appliances. }\end{array}$ \\
\hline DATE PROGRAM STARTED: & 1981 \\
\hline FIELDS OF TESTING: & $\begin{array}{ll}\text { Acoustic and Vibration Testing } & \text { Mechanical Testing } \\
\square \text { Biological Testing } & \square \text { Metrology } \\
\text { Chemical Testing } & \square \text { Nondestructive Testing } \\
\bigotimes \text { Electrical Testing } & \square \text { Optics and Photometry } \\
\square \text { Ionizing Radiation } & \square \text { Thermal Testing } \\
\square \text { Other } & \end{array}$ \\
\hline $\begin{array}{l}\text { CATEGORIES OF PRODUCTS } \\
\text { COVERED BY THE PROGRAM: }\end{array}$ & $\begin{array}{l}\text { Products, materials and assemblies are required by the Ohio Basic } \\
\text { Building code to be approved, labeled or grade-marked for use in } \\
\text { building construction. }\end{array}$ \\
\hline $\begin{array}{l}\text { NUMBER OF LABS ACCREDITED/ } \\
\text { APPROVED: }\end{array}$ & 9 . \\
\hline $\begin{array}{l}\text { ACCREDITATION/APPROVAL } \\
\text { VALID FOR: }\end{array}$ & Until expiration date listed on their certification. \\
\hline NUMBER OF ASSESSORS: & 7 . \\
\hline $\begin{array}{l}\text { MANDATORY ASSESSOR } \\
\text { QUALIFICATIONS : }\end{array}$ & \begin{tabular}{|l|l}
$\square$ No & Yes, Appendix P of Code.
\end{tabular} \\
\hline PARTICIPATION FEE : & \begin{tabular}{l|l}
$\square$ No & $\square$ Yes
\end{tabular} \\
\hline FEE SCHEDULE AVAILABLE: & $\square$ No \\
\hline PROGRAM HAS AN APPEALS PROCEDURE : & \begin{tabular}{|l|l}
$\square$ No & Yes, available upon request.
\end{tabular} \\
\hline PROGRAM HAS A LOGO: & 凶 No \\
\hline $\begin{array}{l}\text { PROGRAM IS OPEN TO } \\
\text { LABORATORIES : }\end{array}$ & $\begin{array}{ll}\text { All U.S. Private Sector } & \text { All U.S. Government } \\
\text { All Foreign Private Sector } & \text { All Foreign Government } \\
\square \text { Other } & \end{array}$ \\
\hline $\begin{array}{l}\text { AVAILABILITY OF PUBLISHED LIST OF } \\
\text { LABORATORIES: }\end{array}$ & \begin{tabular}{|l|l}
$\square$ No & \es, available upon request.
\end{tabular} \\
\hline $\begin{array}{l}\text { AVAILABILITY OF ASSESSMENT } \\
\text { CRITERIA/PROCEDURES : }\end{array}$ & Available through the Ohio Board of Building Standards. \\
\hline
\end{tabular}




\begin{tabular}{|c|c|}
\hline $\begin{array}{l}\text { APPROVED/ACCREDITED LABORATORY } \\
\text { MANDATED REQUIREMENTS: }\end{array}$ & $\begin{array}{l}\text { Be a legal entity } \\
\square \text { Be financially stable } \\
\text { Be independent of mfrs./suppliers of products tested. } \\
\text { Have an effective quality system } \\
\text { Have procedures to prevent conflicts-of-interest } \\
\square \text { Have a document control system } \\
\square \text { Have a contract review process } \\
\text { Have procedures for sub-contracting tests and calibrations } \\
\square \text { Have a documented procurement process } \\
\square \text { Have a complaints/appeals process } \\
\bigotimes \text { Have a system to control nonconforming testing and/or calibrations } \\
\bigotimes \text { Have a corrective/preventive action process } \\
\bigotimes \text { Have an effective recordkeeping process } \\
\square \text { Have documented record retention requirements } \\
\text { Conduct internal audits of its quality system } \\
\bigotimes \text { Have laboratory management review results of internal audits } \\
\text { Have qualified personnel } \\
\text { Have laboratory measurements traceable to national standards } \\
\square \text { Use effective sampling techniques } \\
\square \text { Have a process for handling/transport of test/calibration items } \\
\square \text { Participate in a proficiency testing program } \\
\bigotimes \text { Have adequate instrumentation facilities and equipment } \\
\text { Ensure adequate equipment maintenance/calibration } \\
\square \text { Attend program laboratory workshops/conferences } \\
\square \text { Maintain other or related accreditations/approvals }\end{array}$ \\
\hline $\begin{array}{l}\text { STANDARD (S) WITH WHICH PROGRAM } \\
\text { CONEORMS: }\end{array}$ & $\square$ No D Don't know \\
\hline $\begin{array}{l}\text { PROGRAM RECOGNITION/MUTUAL } \\
\text { RECOGNITION ARRANGEMENTS : }\end{array}$ & None. \\
\hline
\end{tabular}




\begin{tabular}{|c|c|}
\hline DEPARTMENT/AGENCY : & $\begin{array}{l}\text { OREGON-THREE AGENCY PROGRAM-DEPARTMENT OF ENVIRONMENTAL } \\
\text { QUALITY, DEPARTMENT OF AGRICULTURE, DEPARTMENT OF } \\
\text { HUAMAN SERVICES (HEALTH DIV.) }\end{array}$ \\
\hline ADDRESS : & $\begin{array}{l}\text { ORELAP Administrator, Oregon State Public Health Laboratory, } \\
1717 \text { SW 10th Avenue, Portland, OR } 97201\end{array}$ \\
\hline & $\begin{array}{l}\text { INTERNET URL: } \\
\text { wWw.deg.state.or.us/lab/ } \\
\text { orelap }\end{array}$ \\
\hline & E-MAIL: Irene.E.Ronning@state.or.us. \\
\hline NAME OF PROGRAM: & Oregon Environmental Laboratory Accreditation Program \\
\hline PROGRAM DESCRIPTION : & $\begin{array}{l}\text { Accredits environental testing laboratories for analysis under EPA } \\
\text { programs, CAA, CUA, RCRA and SDWA. }\end{array}$ \\
\hline DATE PROGRAM STARTED: & $\begin{array}{l}\text { Recognized accrediting authority since } 02 / 00 ; \text { began lab } \\
\text { accreditations on } 01 / 01 .\end{array}$ \\
\hline FIELDS OF TESTING: & $\begin{array}{ll}\square \text { Acoustic and Vibration Testing } & \square \text { Mechanical Testing } \\
\bigotimes \text { Biological Testing } & \square \text { Metrology } \\
\bigotimes \text { Chemical Testing } & \square \text { Nondestructive Testing } \\
\square \text { Electrical Testing } & \square \text { Optics and Photometry } \\
\square \text { Ionizing Radiation } & \square \text { Thermal Testing } \\
\square \text { Other } & \end{array}$ \\
\hline $\begin{array}{l}\text { CATEGORIES OF PRODUCTS } \\
\text { COVERED BY THE PROGRAM: }\end{array}$ & Environmental samples. \\
\hline $\begin{array}{l}\text { NUMBER OF LABS ACCREDITED/ } \\
\text { APPROVED: }\end{array}$ & 3 as of 01/01, with about 50 more in process. \\
\hline $\begin{array}{l}\text { ACCREDITATION/APPROVAL } \\
\text { VALID FOR: }\end{array}$ & 1 year. \\
\hline NUMBER OF ASSESSORS: & 5, plus as many third parties approved. \\
\hline $\begin{array}{l}\text { MANDATORY ASSESSOR } \\
\text { QUALIFICATIONS : }\end{array}$ & \begin{tabular}{|l|l}
$\square$ No & $\begin{array}{l}\text { Yes, a bachelor's degree in appropriate } \\
\text { scientific discipline, lab experience, } \\
\text { assessor training courses \& joint } \\
\text { inspections prior to performing on site } \\
\text { alone. }\end{array}$ \\
\end{tabular} \\
\hline PARTICIPATION FEE : & 凶 Yes, available on website and upon request. \\
\hline FEE SCHEDULE AVAILABLE: & 区 Yes, available on website and upon request. \\
\hline PROGRAM HAS AN APPEALS PROCEDURE: & $\begin{array}{l}\text { \ Yes, to the Department of Human Services, Health } \\
\text { Division. }\end{array}$ \\
\hline PROGRAM HAS A LOGO: & 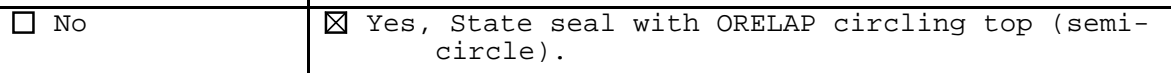 \\
\hline $\begin{array}{l}\text { PROGRAM IS OPEN TO } \\
\text { LABORATORIES : }\end{array}$ & $\begin{array}{ll}\text { All U.S. Private Sector } & \text { All U.S. Government } \\
\text { All Foreign Private Sector } & \text { All Foreign Government } \\
\text { 冈 Other municipal and county labs. } & \end{array}$ \\
\hline $\begin{array}{l}\text { AVAILABILITY OF PUBLISHED LIST OF } \\
\text { LABORATORIES: }\end{array}$ & $\begin{array}{l}\bigotimes \text { Yes, the list will be on website in the near } \\
\text { future. }\end{array}$ \\
\hline $\begin{array}{l}\text { AVAILABILITY OF ASSESSMENT } \\
\text { CRITERIA/PROCEDURES : }\end{array}$ & NELAC 1999 standards, on NELAC website at www.epa.gov/ttn/nelac. \\
\hline
\end{tabular}




\begin{tabular}{|c|c|}
\hline $\begin{array}{l}\text { APPROVED/ACCREDITED LABORATORY } \\
\text { MANDATED REQUIREMENTS: }\end{array}$ & 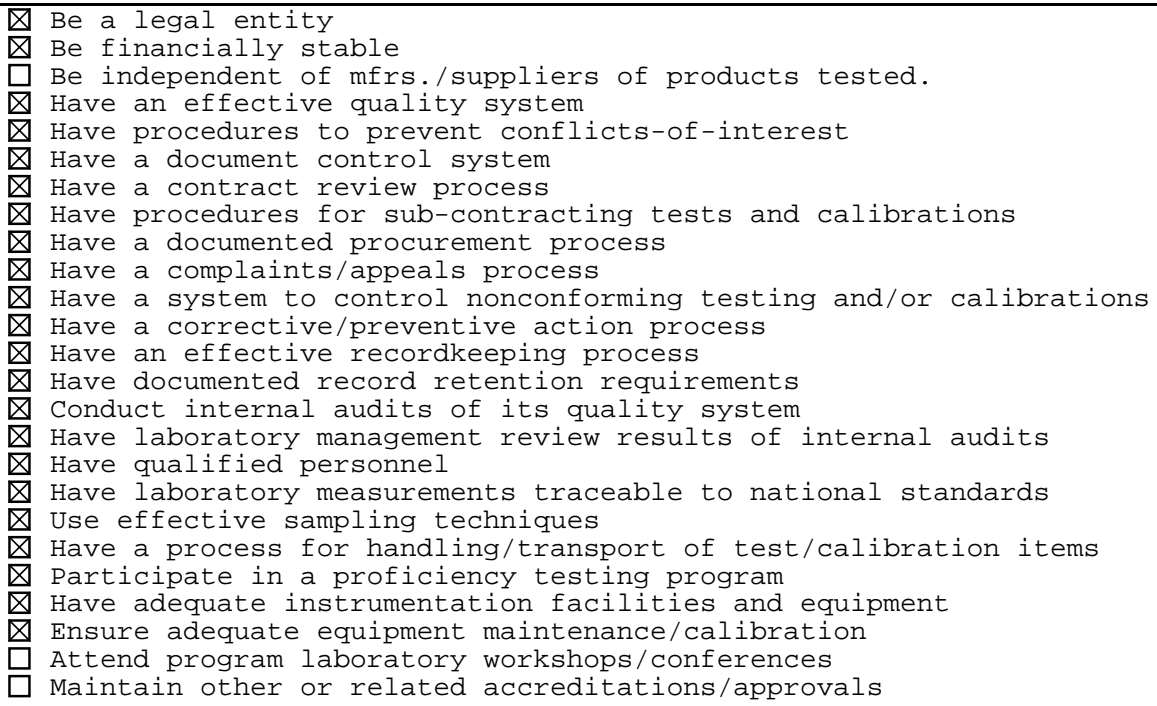 \\
\hline $\begin{array}{l}\text { STANDARD (S) WITH WHICH PROGRAM } \\
\text { CONFORMS : }\end{array}$ & 区 Yes, NELAC 1999 standards. \\
\hline $\begin{array}{l}\text { PROGRAM RECOGNITION/MUTUAL } \\
\text { RECOGNITION ARRANGEMENTS : }\end{array}$ & Other NELAP recognized accrediting authorities. \\
\hline
\end{tabular}




\begin{tabular}{|c|c|}
\hline DEPARTMENT/AGENCY : & $\begin{array}{l}\text { VIRGINIA, CITY OF RICHMOND, DEPARTMENT OF PUBLIC } \\
\text { UTILITIES; ENVIRONMENTAL MANAGEMENT SERVICES DIV. }\end{array}$ \\
\hline ADDRESS : & 600 East Broad Street, Room No. 821, Richmond, Virginia 23219 \\
\hline & PHONE : $804-646-5183$ \\
\hline & E-MAIL: wichserrecl.richmond.va.us \\
\hline NAME OF PROGRAM: & Regulatory Compliance and Special Studies \\
\hline PROGRAM DESCRIPTION: & $\begin{array}{l}\text { Acceptance of organizations judged by the Chief Utility Engineer to } \\
\text { be a recognized special studies body for the inspection and approval } \\
\text { of products and services. }\end{array}$ \\
\hline DATE PROGRAM STARTED: & April 1991. \\
\hline FIELDS OF TESTING: & $\begin{array}{ll}\square \text { Acoustic and Vibration Testing } & \square \text { Mechanical Testing } \\
\text { Biological Testing } & \square \text { Metrology } \\
\bigotimes \text { Chemical Testing } & \square \text { Nondestructive Testing } \\
\square \text { Electrical Testing } & \square \text { Optics and Photometry } \\
\square \text { Ionizing Radiation } & \square \text { Thermal Testing } \\
\square \text { Other } & \end{array}$ \\
\hline $\begin{array}{l}\text { CATEGORIES OF PRODUCTS } \\
\text { COVERED BY THE PROGRAM: }\end{array}$ & Drinking water; wastewater; utility infrastructure. \\
\hline $\begin{array}{l}\text { NUMBER OF LABS ACCREDITED/ } \\
\text { APPROVED: }\end{array}$ & 3 . \\
\hline $\begin{array}{l}\text { ACCREDITATION/APPROVAL } \\
\text { VALID FOR: }\end{array}$ & $3-5$ years. \\
\hline NUMBER OF ASSESSORS: & $2-3$ \\
\hline $\begin{array}{l}\text { MANDATORY ASSESSOR } \\
\text { QUALIFICATIONS : }\end{array}$ & 囚 Yes B.S./M.S. plus experience. \\
\hline PARTICIPATION FEE: & $\square$ Yes \\
\hline FEE SCHEDULE AVAILABLE: & $\square$ Yes \\
\hline PROGRAM HAS AN APPEALS PROCEDURE: & $\square$ Yes \\
\hline PROGRAM HAS A LOGO: & $\square$ Yes \\
\hline $\begin{array}{l}\text { PROGRAM IS OPEN TO } \\
\text { LABORATORIES : }\end{array}$ & $\begin{array}{l}\text { 冈 All U.S. Private Sector } \\
\square \text { All Foreign Private Sector } \\
\square \text { Other }\end{array}$ \\
\hline $\begin{array}{l}\text { AVAILABILITY OF PUBLISHED LIST OF } \\
\text { DIRECTORIES AVAILABLE: }\end{array}$ & $\square$ Yes \\
\hline $\begin{array}{l}\text { AVAILABILITY OF ASSESSMENT } \\
\text { CRITERIA/PROCEDURES : }\end{array}$ & \\
\hline
\end{tabular}




\begin{tabular}{|c|c|}
\hline $\begin{array}{l}\text { APPROVED/ACCREDITED LABORATORY } \\
\text { MANDATED REQUIREMENTS: }\end{array}$ & 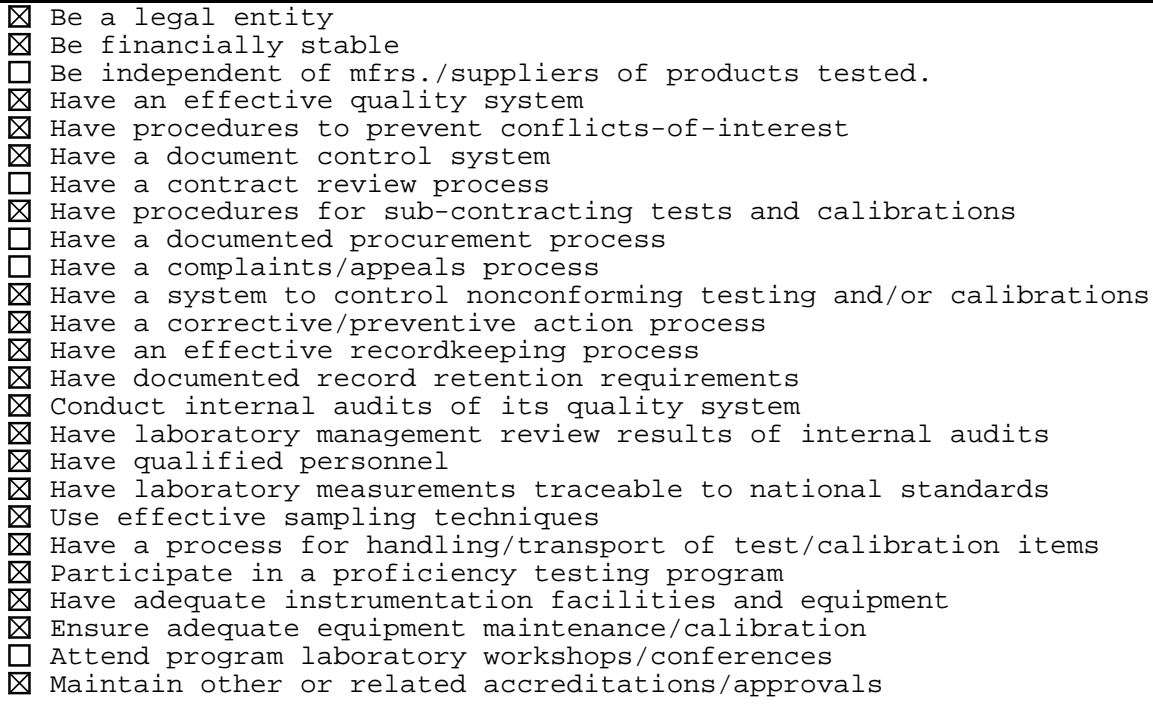 \\
\hline $\begin{array}{l}\text { STANDARD (S) WITH WHICH PROGRAM } \\
\text { CONFORMS: }\end{array}$ & $\square$ No $\triangle$ Don't know \\
\hline $\begin{array}{l}\text { PROGRAM RECOGNITION/MUTUAL } \\
\text { RECOGNITION ARRANGEMENTS : }\end{array}$ & None. \\
\hline
\end{tabular}


DATE RECEIVED: 08/01

\begin{tabular}{|c|c|}
\hline DEPARTMENT/AGENCY : & WASHINGTON STATE, DEPARTMENT OF LABOR AND INDUSTRIES \\
\hline ADDRESS : & P.O. Box 44460, Olympia, WA 98504-4460 \\
\hline & PHONE : $360-902-5249$ \\
\hline & E-MAIL: Fulr235@lni.wa.gov \\
\hline NAME OF PROGRAM: & Accreditation as an Electrical Testing Laboratory. \\
\hline PROGRAM DESCRIPTION: & $\begin{array}{l}\text { Approval of laboratories to perform field evaluations or list } \\
\text { electrical equipment that will be acceptable in the State of } \\
\text { Washington. }\end{array}$ \\
\hline DATE PROGRAM STARTED: & 1935. \\
\hline FIELDS OF TESTING: & $\begin{array}{ll}\square \text { Acoustic and Vibration Testing } & \square \text { Mechanical Testing } \\
\square \text { Biological Testing } & \square \text { Metrology } \\
\square \text { Chemical Testing } & \square \text { Nondestructive Testing } \\
\text { Electrical Testing } & \square \text { Optics and Photometry } \\
\square \text { Ionizing Radiation } & \square \text { Thermal Testing } \\
\square \text { Other } & \end{array}$ \\
\hline $\begin{array}{l}\text { CATEGORIES OF PRODUCTS } \\
\text { COVERED BY THE PROGRAM: }\end{array}$ & Not available. \\
\hline $\begin{array}{l}\text { NUMBER OF LABS ACCREDITED/ } \\
\text { APPROVED: }\end{array}$ & 9 as of June 15, 2001. \\
\hline $\begin{array}{l}\text { ACCREDITATION/APPROVAL } \\
\text { VALID FOR: }\end{array}$ & $\begin{array}{l}\text { Valid for } 5 \text { years for OSHA Nationally Recognized Testing Laboratories } \\
\text { (NRTLs), and one year for non-NRTL's. }\end{array}$ \\
\hline NUMBER OF ASSESSORS: & 8. \\
\hline $\begin{array}{l}\text { MANDATORY ASSESSOR } \\
\text { QUALIFICATIONS : }\end{array}$ & \begin{tabular}{c|c} 
No & $\begin{array}{c}\text { Yes, as prescribed by RCW 19.28 or WAC 296-46A, } \\
296-402 \mathrm{~A} . \text { Qualifications available upon } \\
\text { request. }\end{array}$
\end{tabular} \\
\hline PARTICIPATION FEE: & Q Yes \\
\hline FEE SCHEDULE AVAILABLE: & $\begin{array}{l}\text { 区 Yes. The Fee Schedule is published on the } \\
\text { inernet } \\
\text { at www.lni.wa.gov/scs/electrical }\end{array}$ \\
\hline PROGRAM HAS AN APPEALS PROCEDURE : & $\begin{array}{c}\text { Q Yes. Appeals are made to the State Electrical } \\
\text { Advisory Board - State of Washington. }\end{array}$ \\
\hline PROGRAM HAS A LOGO: & $\square$ Yes \\
\hline $\begin{array}{l}\text { PROGRAM IS OPEN TO } \\
\text { LABORATORIES : }\end{array}$ & $\begin{array}{ll}\text { All U.S. Private Sector } & \square \text { All U.S. Government } \\
\text { All Foreign Private Sector } & \square \text { All Foreign Government } \\
\square \text { Other } & \end{array}$ \\
\hline $\begin{array}{l}\text { AVAILABILITY OF PUBLISHED LIST OF } \\
\text { DIRECTORIES AVAILABLE: }\end{array}$ & $\begin{array}{l}\text { 囚 Yes. It's available on the website at } \\
\text { www.lni.wa.gov/scs/electrical. }\end{array}$ \\
\hline $\begin{array}{l}\text { AVAILABILITY OF ASSESSMENT } \\
\text { CRITERIA/PROCEDURES: }\end{array}$ & It's available on the website at www.lni.wa..gov/scs/electrical. \\
\hline
\end{tabular}




\begin{tabular}{|c|c|}
\hline $\begin{array}{l}\text { APPROVED/ACCREDITED LABORATORY } \\
\text { MANDATED REQUIREMENTS: }\end{array}$ & 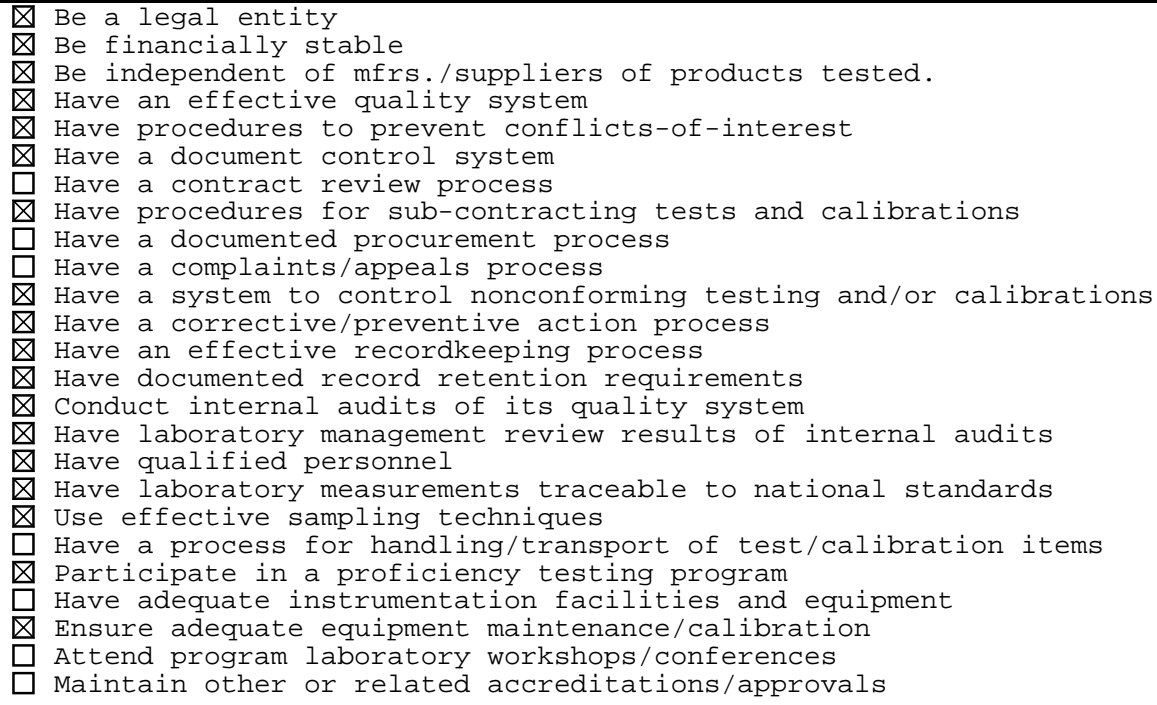 \\
\hline $\begin{array}{l}\text { STANDARD (S) WITH WHICH PROGRAM } \\
\text { CONFORMS : }\end{array}$ & \begin{tabular}{|l} 
Yes, those of the International \\
Standards Organization (ISO), and \\
the Department of Labor's \\
Occupational Safety and Health \\
Administration's (OSHA) Nationally \\
Recognized Testing Laboratory \\
(NRTL) Program.
\end{tabular} \\
\hline $\begin{array}{l}\text { PROGRAM RECOGNITION/MUTUAL } \\
\text { RECOGNITION ARRANGEMENTS: }\end{array}$ & Not applicable. \\
\hline
\end{tabular}




\begin{tabular}{|c|c|}
\hline DEPARTMENT/AGENCY : & $\begin{array}{l}\text { WYOMING DEPARTMENT OF ENVIRONMENTAL QUALITY, LUST/AUST } \\
\text { PROGRAM THROUGH AMERICAN ASSOCIATION FOR LABORATORY } \\
\text { ACCREDITATION [A2LA] }\end{array}$ \\
\hline ADDRESS : & $\begin{array}{l}\text { Laust Remidiation Program Principle, } 122 \text { W 25th Street, 4W, Herschler } \\
\text { Bldg., Cheyenne, WY } 82002\end{array}$ \\
\hline & PHONE : $307-777-7077$ \\
\hline & E-MAIL: khalvo@state.wy.us \\
\hline NAME OF PROGRAM: & A2LA laboratory accreditation program for lUST/AUST Program. \\
\hline PROGRAM DESCRIPTION: & Laboratory Accreditation. \\
\hline DATE PROGRAM STARTED: & 2001 \\
\hline FIELDS OF TESTING: & $\begin{array}{ll}\square \text { Acoustic and Vibration Testing } & \square \text { Mechanical Testing } \\
\square \text { Biological Testing } & \square \text { Metrology } \\
\square \text { Chemical Testing } & \square \text { Nondestructive Testing } \\
\square \text { Electrical Testing } & \square \text { Optics and Photometry } \\
\square \text { Ionizing Radiation } & \square \text { Thermal Testing } \\
\bigotimes \text { Other Environmental } & \end{array}$ \\
\hline $\begin{array}{l}\text { CATEGORIES OF PRODUCTS } \\
\text { COVERED BY THE PROGRAM: }\end{array}$ & Available through A2LA. \\
\hline $\begin{array}{l}\text { NUMBER OF LABS ACCREDITED/ } \\
\text { APPROVED: }\end{array}$ & Available through A2LA. \\
\hline $\begin{array}{l}\text { ACCREDITATION/APPROVAL } \\
\text { VALID FOR: }\end{array}$ & Available through A2LA. \\
\hline NUMBER OF ASSESSORS: & Available through A2LA. \\
\hline $\begin{array}{l}\text { MANDATORY ASSESSOR } \\
\text { QUALIFICATIONS : }\end{array}$ & 区 Yes, A2LA requirements available from A2LA. \\
\hline PARTICIPATION FEE : & 凶 Yes, available from A2LA. \\
\hline FEE SCHEDULE AVAILABLE: & 囚 Yes, available from A2LA. \\
\hline PROGRAM HAS AN APPEALS PROCEDURE : & 区 Yes, available through A2LA. \\
\hline PROGRAM HAS A LOGO: & $\square$ Yes \\
\hline $\begin{array}{l}\text { PROGRAM IS OPEN TO } \\
\text { LABORATORIES : }\end{array}$ & $\begin{array}{l}\text { Q All U.S. Private Sector } \\
\text { All Foreign Private Sector } \\
\square \text { Other }\end{array}$ \\
\hline $\begin{array}{l}\text { AVAILABILITY OF PUBLISHED LIST OF } \\
\text { LABORATORIES : }\end{array}$ & $\square$ Yes \\
\hline $\begin{array}{l}\text { AVAILABILITY OF ASSESSMENT } \\
\text { CRITERIA/PROCEDURES : }\end{array}$ & Available through A2LA. \\
\hline
\end{tabular}




\begin{tabular}{|c|c|}
\hline $\begin{array}{l}\text { APPROVED/ACCREDITED LABORATORY } \\
\text { MANDATED REQUIREMENTS: }\end{array}$ & $\begin{array}{l}\text { Be a legal entity } \\
\text { Be financially stable } \\
\text { Be independent of mfrs./suppliers of products tested. } \\
\text { Have an effective quality system } \\
\text { Have procedures to prevent conflicts-of-interest } \\
\text { Have a document control system } \\
\text { Have a contract review process } \\
\text { Have procedures for sub-contracting tests and calibrations } \\
\text { Have a documented procurement process } \\
\text { Have a complaints/appeals process } \\
\text { Have a system to control nonconforming testing and/or calibrations } \\
\text { Have a corrective/preventive action process } \\
\text { Have an effective recordkeeping process } \\
\text { Have documented record retention requirements } \\
\text { H Conduct internal audits of its quality system } \\
\text { Have laboratory management review results of internal audits } \\
\text { Have qualified personnel } \\
\text { Have laboratory measurements traceable to national standards } \\
\text { Use effective sampling techniques } \\
\text { Have a process for handling/transport of test/calibration items } \\
\text { Participate in a proficiency testing program } \\
\text { Have adequate instrumentation facilities and equipment } \\
\text { H Ensure adequate equipment maintenance/calibration } \\
\text { Httend program laboratory workshops/conferences } \\
\text { Haintain other or related accreditations/approvals }\end{array}$ \\
\hline $\begin{array}{l}\text { STANDARD (S) WITH WHICH PROGRAM } \\
\text { CONFORMS : }\end{array}$ & 区 Yes, available through A2LA. \\
\hline $\begin{array}{l}\text { PROGRAM RECOGNITION/MUTUAL } \\
\text { RECOGNITION ARRANGEMENTS : }\end{array}$ & Nor \\
\hline
\end{tabular}




\section{APPENDIX I - INDICES}

INDEX

PAGE

State / Local/Municipal Accreditation/Designation

SYSTEMS

64

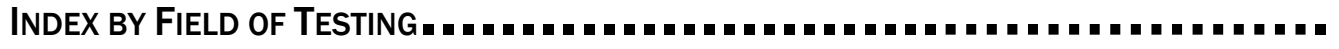

67

INDEX BY PRODUCTS TESTED

69 


\section{INDEX OF STATE/LOCAL/MUNICIPAL ACCREDITATION/DESIGNATION SYSTEMS}

$\underline{\text { STATE }}$

$\underline{\text { PAGES }}$

Alabama Department of EnVIRONMENTAL MANAGEMENt [ADEM] 9

California State Department of Health Services 11

CALIFORNIA, CITY OF LOS ANGELES 13

FLORIDA DIVISION OF STATE FIRE MARSHAL

FlORIDA - MIAMI- DADE PRODUCT CONTROL DIVISION

Georgia EnVIROnMental Protection Division

KANSAS - DEPARTMENT OF HEALTH \& ENVIRONMENT LABORATORY IMPROVEMENT PROGRAM (\# 1 )

KANSAS - DEPARTMENT OF HEALTH \& ENVIRONMENT -

LABORATORY IMPROVEMENT PROGRAM (\#2)

KANSAS - DEPARTMENT OF HEALTH \& ENVIRONMENT -

LABORATORY IMPROVEMENT PROGRAM (\#3)

KANSAS - DEPARTMENT OF HEALTH \& ENVIRONMENT LABORATORY IMPROVEMENT PROGRAM (\#4)

KENTUCKY DEPARTMENT FOR ENVIRONMENTAL PROTECTION,

DRINKING WATER BRANCH

LOUISIANA DEPARTMENT OF ENVIRONMENTAL QUALITY

LOUISIANA DEPARTMENT OF HEALTH \& HOSPITALS,

OfFICE Of PUBLIC HEALTH

MINNESOTA DEPARTMENT OF AGRICULTURE,

AGRONOMY/PLANT PROTECTION INCIDENT RESPONSE PROGRAM

MINNESOTA DEPARTMENT OF AGRICULTURE,

LABORATORY SERVICES DIVISION

NeW HAMPSHIRE DepartMENT OF ENVIRONMENTAL SERVICE

NEW JeRsey DepartMent of ENVIRONMENTAL PROTECTION 
New York State Department Of Agriculture AND MARKETS,

DIVISION OF MILK CONTROL --_--_-- 45

NEW YORK - THE CITY OF NEW YORK - DePARTMENT OF BUILDINGS MATERIALS AND EQUIPMENT ACCEPTANCE DIVISION [MEA]

North Carolina Department of Insurance, Office Of State Fire Marshal AND N.C. BUILDING CODE COUNCIL -

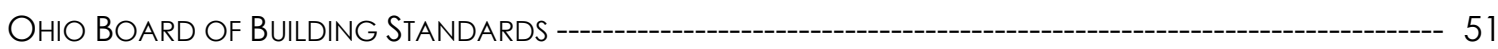

Ohio, City of Cleveland, Department of Community Development, DIVISION OF BUILDING AND HOUSING --_ 53

OREGON - THREE-AGENCY PROGRAM - DEPARTMENT OF ENVIRONMENTAL QUALITY,

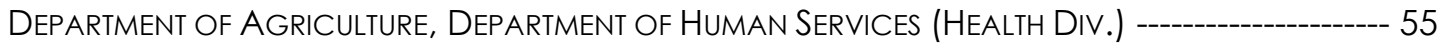

VIRGINIA, City Of Richmond, DepartMent OF PubliC Utilities;

ENVIRONMENTAL MANAGEMENT SERVICES DIV. --_-_- 57

WASHINGTON STATE, DEPARTMENT OF LABOR AND INDUSTRIES -- 59

WYOMING DEPARTMENT OF ENVIRONMENTAL QUALITY, LUST/AUST PROGRAM

THROUGH AMERICAN ASSOCIATION FOR LABORATORY ACCREDITATION [A2LA] ----------------- 61 


\section{INDEX BY FIELD OF TESTING7}

\begin{tabular}{|c|c|c|}
\hline & FIELD OF TESTING 8 & PAGE \\
\hline $5.1 \quad$ & Acoustics and Vibration Testing & $11,51,53$ \\
\hline $5.2 \mathrm{I}$ & Biological Testing & $11,21,23,25,27,29,31,33,37,39,41,47,55,57$ \\
\hline $5.3 \mathrm{C}$ & Chemical Testing & $\begin{array}{l}9,11,15,21,23,25,27,29,31,33,35,37,39,41,47, \\
51,53,55,57\end{array}$ \\
\hline $5.4 \quad 1$ & Electrical Testing & $13,15,47,49,51,53,59$ \\
\hline $5.5 \mathrm{I}$ & Ionizing Radiation & $11,31,39,41$ \\
\hline $5.6 \quad 1$ & Mechanical Testing & $13,47,49,51,53$ \\
\hline 5.7. 1 & Metrology & 47 \\
\hline $5.8 \mathrm{I}$ & Nondestructive Testing & $51,53,57$ \\
\hline $5.9 \quad \mathrm{c}$ & Optics and Photometry & \\
\hline 5.107 & Thermal Testing & $15,41,47,51,53$ \\
\hline Other: & $\begin{array}{l}\text { - Sanitation } \\
\text { - Hurricane Protection } \\
\text { - Environmental } \\
\text { - Whole Effluent Toxicity } \\
\text { - Regulatory Certification of Drinking Water } \\
\text { Compliance Analysis } \\
\text { - Milk Testing }\end{array}$ & $\begin{array}{l}\text { - } 11 \\
-17 \\
-19,61 \\
\cdot-39 \\
\text { - } 43 \\
\text { - } 45\end{array}$ \\
\hline
\end{tabular}

${ }^{7}$ Includes requirements for New Mexico and Wyoming. These states do not accredit laboratories directly, but rely on private sector laboratory accreditation programs. ${ }^{8}$ Categories from E 1224-94 Standard Guide for Categorizing Fields of Testing for Laboratory Accreditation Purposes "5. Part One - Testing Technology (Fields of Testing)" 


\begin{tabular}{|c|c|}
\hline Products/Services Tested 9 & PAGE \\
\hline \multicolumn{2}{|l|}{ Acoustics and Vibration Testing: } \\
\hline - 6.28 Construction Materials & 51,53 \\
\hline - Health Services & 11 \\
\hline \multicolumn{2}{|l|}{ Biological Testing: } \\
\hline - Air & 23,41 \\
\hline - $\quad$ Breath Alcohol & 21 \\
\hline - Drinking Water & $9,11,29,33,41,43,57$ \\
\hline - $\quad 6.36$ Drugs & 27 \\
\hline - Environmental Biology & $19,31,41,55$ \\
\hline - Hazardous Waste & 11,41 \\
\hline - 5.25 Human Studies & 25 \\
\hline - Milk & $37,43,45$ \\
\hline - $\quad$ Pesticides Residues & 11 \\
\hline - Shellfish Sanitation & 11 \\
\hline - Soil & 23,41 \\
\hline - Wastewater & $11,39,41,57$ \\
\hline \multicolumn{2}{|l|}{ Chemical Testing: } \\
\hline - Arson Investigations Forensic & 15 \\
\hline - Breath Alcohol & 21 \\
\hline - $\quad 6.28$ Construction Materials & $17,43,47,49,51,53$ \\
\hline - Drinking Water & $9,23,29,33,41,43,57$ \\
\hline - $\quad 6.36$ Drugs & 27 \\
\hline - $\quad$ 6.45 Environmental Testing & $19,31,41,55,61$ \\
\hline - Hazardous Waste & 11,41 \\
\hline - Human Studies & 25 \\
\hline - Insecticides Agricultural & 35 \\
\hline - Milk & 37 \\
\hline - $\quad$ Pesticides Residues & 11 \\
\hline - Shellfish Sanitation & 11 \\
\hline
\end{tabular}




\begin{tabular}{|c|c|}
\hline - Waste Water & $11,23,39,41,57$ \\
\hline \multicolumn{2}{|l|}{ Electrical Testing: } \\
\hline - Arson Investigations Forensic & 15 \\
\hline - 6.28 Construction Materials & $17,51,53,59$ \\
\hline - $\quad$ Construction Materials \& Equipment & $13,47,49,59$ \\
\hline \multicolumn{2}{|l|}{ Ionizing Radiation: } \\
\hline - Environmental Testing & 31,41 \\
\hline - Health Services & 11,39 \\
\hline \multicolumn{2}{|l|}{ Mechanical Testing: } \\
\hline - 6.28 Construction Materials & 17 \\
\hline - Environmental Testing & 61 \\
\hline $\begin{array}{l}\text { - Structural Steel, wood, concrete, roofing, glass, } \\
\text { insulation, etc. }\end{array}$ & $13,47,49$ \\
\hline \multicolumn{2}{|l|}{ Metrology: } \\
\hline - Construction Testing Equipment & $43,51,53$ \\
\hline \multicolumn{2}{|l|}{ Nondestructive Testing: } \\
\hline - 6.28 Construction Materials & $51,53,57$ \\
\hline \multicolumn{2}{|l|}{ Optics and Photometry: } \\
\hline \multicolumn{2}{|l|}{ Thermal Testing: } \\
\hline - Arson Investigations Forensic & 15 \\
\hline - 6.28 Construction Materials & $17,47,51,53$ \\
\hline
\end{tabular}




\section{APPENDIX II: FORMAT USED FOR EACH ENTRY}

DATE RECEIVED: Date Material Received from State/Local Government Agency

\begin{tabular}{|c|c|}
\hline Department/Agency: & $\begin{array}{l}\text { The name of the responsible office/agency/ department, as well as its } \\
\text { address, telephone, telex, and fax numbers, internet URL address, and } \\
\text { e-mail address (if available). }\end{array}$ \\
\hline Name of Program: & $\begin{array}{l}\text { Name of the program or part of the program responsible for laboratory } \\
\text { accreditation or designation. }\end{array}$ \\
\hline Program Description: & Brief description of the program's mission, scope, and intent. \\
\hline Date Program was Started: & The date the program was established. \\
\hline Fields of Testing: & $\begin{array}{l}\text { The fields of testing covered by the program as listed in ASTM E 1224- } \\
94 \text { Standard Guide for Categorizing Fields of Capability for } \\
\text { Laboratory Accreditation Purposes and/or other fields as indicated by } \\
\text { the agency. }\end{array}$ \\
\hline Categories of Products Covered by the Program: & $\begin{array}{l}\text { The products, services, installations, etc., which laboratories are } \\
\text { accredited or designated to test, examine, inspect, etc. }\end{array}$ \\
\hline Number of Labs Accredited/Approved: & The number of labs accredited as of a specified date. \\
\hline How long is accreditation/approval valid? & The period of time for which an accreditation or designation is valid. \\
\hline How often is accreditation/approval reissued? & $\begin{array}{l}\text { The period of time after which all or part of the } \\
\text { accreditation/designation process must be repeated. }\end{array}$ \\
\hline How many assessors do you have? & $\begin{array}{l}\text { The number of individuals/organizations that conduct or are eligible to } \\
\text { conduct laboratory accreditation/designation assessments. }\end{array}$ \\
\hline $\begin{array}{l}\text { Are there any mandatory assessors' } \\
\text { qualifications? } \\
\text { Yes__ No__ }\end{array}$ & $\begin{array}{l}\text { Any educational, training and/or experience requirements for the } \\
\text { individuals/organizations as a prerequisite for performing laboratory } \\
\text { accreditation/designation assessments. }\end{array}$ \\
\hline If Yes, how may a copy be obtained? & $\begin{array}{l}\text { Information on how a copy of any mandatory assessor qualification } \\
\text { standards or requirements may be obtained. }\end{array}$ \\
\hline Is there a fee to participate? Yes_ No & $\begin{array}{l}\text { Charges or cost reimbursement fees required from a laboratory seeking } \\
\text { accreditation/ designation. }\end{array}$ \\
\hline $\begin{array}{l}\text { Is there fee schedule? Yes_No _ } \\
\text { If Yes, how may a copy be obtained? }\end{array}$ & $\begin{array}{l}\text { Existence of a formal schedule of fees and other charges for } \\
\text { accreditation/designation services. If such as schedule exists, how a } \\
\text { copy can be obtained. }\end{array}$ \\
\hline $\begin{array}{l}\text { Can accreditation/approval decisions be } \\
\text { appealed? } \\
\text { Yes__ No__ If Yes, to whom is the appeal made? }\end{array}$ & $\begin{array}{l}\text { Existence of an appeals process/procedures used to review appeals and } \\
\text { other complaints regarding actions taken by the program or by the } \\
\text { designated/accredited laboratories. If such a process exists, the name of } \\
\text { the individual or body to whom such an appeal may be made. }\end{array}$ \\
\hline
\end{tabular}




\begin{tabular}{|c|c|}
\hline $\begin{array}{l}\text { Logo: Does your program have a logo or mark? } \\
\text { Yes__No__ If Yes, please describe. }\end{array}$ & $\begin{array}{l}\text { Determination of the existence of a logo, mark or similar symbol, } \\
\text { which identifies the accreditation/designation status of a laboratory. If } \\
\text { such a logo, mark or similar symbol exists, the terms under which } \\
\text { accredited/designated laboratories may use it. }\end{array}$ \\
\hline $\begin{array}{l}\text { Is your program open to (Check as appropriate: } \\
\text { _All U.S. Private Sector Laboratories } \\
\text { _All U.S. Government Laboratories } \\
\text { _All Foreign Private Sector Laboratories } \\
\text { _ All Foreign Government Laboratories } \\
\text { _ Other: (Please List) }\end{array}$ & $\begin{array}{l}\text { Whether the accreditation or designation system is open to U.S. } \\
\text { independent testing labs; profit and/or nonprofit private sector labs; } \\
\text { federal labs; other state, local and municipal government labs; and/or } \\
\text { private or governmental foreign labs. }\end{array}$ \\
\hline $\begin{array}{l}\text { Do you publish lists or directories of } \\
\text { laboratories? } \\
\text { Yes_No_If Yes, please provide a copy or title } \\
\text { and information on availability: }\end{array}$ & $\begin{array}{l}\text { Availability of directories/lists of accredited or designated laboratories } \\
\text { or directories/lists of approved or acceptable products. If copies could } \\
\text { not be provided with response, the title of the publication with } \\
\text { information on how it may be obtained. }\end{array}$ \\
\hline $\begin{array}{l}\text { Where can your assessment criteria/procedures } \\
\text { be obtained? }\end{array}$ & $\begin{array}{l}\text { The documents containing the procedures or criteria used by the } \\
\text { accrediting agency, (including referenced international, federal and } \\
\text { non-government standards, specifications and test methods) that define } \\
\text { conditions/ requirements for accreditation or designation and } \\
\text { information on how such documents can be obtained. }\end{array}$ \\
\hline $\begin{array}{l}\text { An approved/accredited laboratory must: (Please } \\
\text { check as appropriate) }\end{array}$ & $\begin{array}{l}\text { Conditions/requirements must be met by accredited/designated laboratories: } \\
\text {-Be A Legal Entity } \\
\text {-Be Financially Stable } \\
\text {-Be Independent Of Manufacturers/Suppliers Of Products Tested } \\
\text {-Have An Effective Quality System } \\
\text {-Have Procedures To Prevent Conflicts-Of-Interest } \\
\text {-Have A Document Control System } \\
\text {-Have A Contract Review Process } \\
\text {-Have Procedures For Sub-Contracting Tests And Calibrations } \\
\text {-Have A Documented Procurement Process } \\
\text {-Have A Complaints/Appeals Process } \\
\text {-Have A System To Control Nonconforming Testing And/Or Calibrations } \\
\text {-Have A Corrective/Preventive Action Process } \\
\text {-Have An Effective Recordkeeping } \\
\text {-Have Documented Record Retention Requirements } \\
\text {-Conduct Internal Audits Of Its Quality System } \\
\text {-Have Laboratory Management Review Results Of Internal Audits } \\
\text {-Have Qualified Personnel } \\
\text {-Have Laboratory Measurements Traceable To National Standards (Where } \\
\text { Applicable) } \\
\text {-Use Effective Sampling Techniques } \\
\text {-Have A Process For Handling/Transport Of Test/Calibration Items } \\
\text {-Participate In A Proficiency Testing Program } \\
\text {-Have Adequate Instrumentation Facilities And Equipment } \\
\text {-Ensure Adequate Equipment } \\
\text {-Maintenance/Calibration } \\
\text {-Attend Program Laboratory Workshops/ Conferences } \\
\text {-Maintain Other Or Related Accreditation's/ Approvals }\end{array}$ \\
\hline
\end{tabular}




\begin{tabular}{|c|c|}
\hline $\begin{array}{l}\text { Are your requirements for laboratories } \\
\text { comparable to those in any other state, national, } \\
\text { or international standard? } \\
\text { Yes_No__Don't Know__ } \\
\text { If Yes, please identify the state(s)/standard: }\end{array}$ & $\begin{array}{l}\text { Comparability of program's requirements to those in specific state, } \\
\text { national or international programs/standards, including the } \\
\text { identification of those programs/standards. }\end{array}$ \\
\hline $\begin{array}{l}\text { Does your program operate in conformance with } \\
\text { any national or international standard: Yes__ } \\
\text { No__Don't Know__ } \\
\text { If yes, please specify the standard: }\end{array}$ & $\begin{array}{l}\text { Conformance of program's requirements to any national or } \\
\text { international standard and the identity of such standard. }\end{array}$ \\
\hline $\begin{array}{l}\text { Is your program recognized by or do you have } \\
\text { any mutual recognition arrangements with any } \\
\text { federal, state (other than your own), foreign } \\
\text { government agency or private sector } \\
\text { organization? (Please identify) }\end{array}$ & $\begin{array}{l}\text { Determination if there are any national, international, private sector or } \\
\text { government mutual recognition arrangements or other recognition that } \\
\text { affects the status of accredited/approved laboratories. If such exists, } \\
\text { identification of each arrangement/recognition. }\end{array}$ \\
\hline
\end{tabular}




\title{
APPENDIX III
}

\section{INFORMATION AND PUBLICATIONS AVAILABLE FROM}

\author{
Standards Services Division \\ National Institute of Standards and Technology \\ Gaithersburg, Maryland 20899-2100 \\ (See last page for ordering information)
}

\section{REPORTS ON THE U.S. STANDARDS SYSTEM}

[Note: A list of U.S. private sector standards developing organizations is available at: http://www.nssn.org/acrodesc.html]

Proceedings of the NIST Centennial Standards Symposium - Standards in the Global Economv: Past, Present, and Future (NIST SP974)

In honor of its $100^{\text {th }}$ anniversary, the National Institute of Standards and Technology sponsored the NIST Centennial Standards Symposium on March 7, 2001. Representatives of the public and private sector celebrated the role that NIST has played in standards in a variety of industry sectors and partnership with numerous organizations. This publication is a compilation of speeches and presentations from multiple speakers on various standards-related topics. Order from NIST by \#PB 2002-101061.

\section{A Guide to Documentary Standards (NISTIR 6802)}

This Guide is intended to provide information on the U.S. standards system, entities within that system, and different types of documentary standards. It includes descriptions of performance and design standards; voluntary consensus standards; defense standards; mandatory standards; National Institute of Justice (NIJ) standards; Federal standards; de facto standards; industry standards; consortia standards; and, international standards. In addition, the Guide includes information on the implementation of the National Technology Transfer and Advance Act (NTTAA) and on the link between standards and conformity assessment. Order from NTIS by \#PB 2001-109032.

\section{The ABC's of Standards-Related Activities in the United States (NBSIR 87-3576)}

This report is an introduction to voluntary standardization, product certification and laboratory accreditation for readers not fully familiar with these topics. It stresses some of the more important aspects of these fields; furnishes the reader with both historical and current information on these topics; describes the importance and impact of the development and use of standards; and serves as background for using available documents and services. Order from NTIS by \#PB 87-224309.

Standards Activities of Organizations in the United States (NIST SP 806, 1996 Edition).

The directory identifies and describes activities of over 700 U.S. public and private sector organizations, which develop, publish, and revise standards; participate in this process; or identify standards and make them available through information centers or distribution channels. The revision covers activities related to both mandatory and voluntary U.S. standards. It also contains a subject index and related listings that cover acronyms and initials, defunct bodies and organizations with name changes. Order from NTIS by \#PB 97-124135/AS.

\section{Toward a National Standards Strategy - Conference Report (NISTIR 6290)}

This more complete report of the September 23, 1998 Summit Proceedings contains full texts, figures, and such background papers as were provided by the speakers; they are presented in the order shown on the agenda. Transcriptions of comments 
and questions from the floor and the ensuing panelist responses are interspersed appropriately. Information about the speakers appears in Appendix A; the list of Conference attendees may be found in Appendix B; and a glossary of acronyms is provided in Appendix C. Order from NTIS by \#PB 99-129629.

\section{Toward a National Standards Strategy - Conference Summary Report (NISTIR 6259)}

On September 23, 1998, the National Institute of Standards and Technology (NIST) and the American National Standards Institute (ANSI) co-hosted a summit conference, 'Toward a National Standards Strategy to Meet Global Needs.' NIST Director Ray Kammer and ANSI President Sergio Mazza co-chaired a program that featured keynote addresses by Deputy Secretary of Commerce Robert Mallet and Dana Mead, Chairman and CEO of Tenneco and World Standards Day Chairman; a luncheon address by Evangelos Vardakas, Director, Directorate B, Legislation and Standardization, Telematics Networks, Directorate General (DG)-III: Industrial Affairs, European Commission; three panels comprised of standards experts from industry and government; and discussions of comments and questions raised by some of the 339 registered participants.

Order from NTIS by \#PB 99-111239.

\section{REPORTS ON THE U.S. CONFORMITY ASSESSMENT SYSTEM}

[Note: additional information on Conformity Assessment is available on the NIST website at: http://ts.nist.gov/ts/htdocs/210/gsig/cainfo.htm]

\section{ABC's of the U.S. Conformity Assessment System (NISTIR 6014)}

This report is designed to provide the reader with an introduction to conformity assessment and information on how the various conformity assessment activities are interlinked. It highlights some of the field's more important aspects and serves as background for using available documents and services. Order from NTIS by \#PB 97-197107.

\section{The ABC'S of Certification Activities in the United States (NBSIR 88-3821)}

This report, a sequel to NBSIR 87-3576, The ABC'S of Standards-Related Activities in the United States, provides an introduction to certification for readers not entirely familiar with this topic. It highlights some of the more important aspects of this field, furnishes the reader with information necessary to make informed purchases, and serves as background for using available documents and services. Order from NTIS by \#PB 88-239793.

\section{The U.S. Certification Svstem from a Government Perspective (NISTIR 6077)}

This report is designed to provide the reader with an introduction to the U.S. certification system from a governmental perspective. It highlights some of the relationships that exist between federal and state agencies and the private sector and discusses some of the history and philosophy behind the U.S. system. Order from NTIS by \#PB 98-104086.

\section{Directory of Federal Government Certification and Related Programs (NIST SP 739, 1999 Edition)}

This fourth edition describes federal government procurement and regulatory programs that may affect product and services in the marketplace. Entries describe the scope and nature of each program, contact points, testing and inspection practices, standards used, methods of identification and enforcement, reciprocal recognition or acceptance of certification and other relevant details. The entries also describe assessment procedures used by federal agencies to provide assurance that the products and services regulated or procured by federal agencies have the required characteristics and/or perform in a specific manner. The methods used by federal agencies to assure conformance can be very different than those traditionally employed by the private sector and by third party certifiers. Order from NTIS by \#PB 99-154825. 
This revision presents information on 122 private sector groups in the United States that engage in product certification activities. Entries describe the type and purpose of each organization, the nature of the activity, a pictorial representation of the organization's mark (if available), products certified, standards used, certification requirements, any accreditation or recognition by a U.S. or foreign private sector or government agency, availability of services, methods of cost determination, and other relevant details. Order from NTIS by \#PB 2001-102896.

Semi-Annual Listing: North American Quality System Registration Organizations (NAQSRO) (NISTIR 6515) This semi-annual listing provides information on organizations that have informed NIST about their quality system registration according to the ASQ Q-9000 series, QS-9000, ISO 14000 (EMS) or equivalent criteria for the United States, Canada and Mexico. Copies are available from the Technical Standards Activities Program at (301) 975-4029, fax: (301) 975-5414; e-mail - tsap@nist.gov. It is also available at the following web site: http://ts.nist.gov/gsip, go to 'Related Information', and click on 'ISO 9000 information.'

\section{Environmental Management Systems Voluntary Project Evaluation Guidance (NISTIR 6120)}

The intent of this document is to provide a framework for the collection of information of value to regulatory agencies and others interested in determining the impact in several key areas of environmental management systems based on ISO 14001. Order from NTIS by \#PB 98-128890.

\section{NVCASE Program Handbook: Procedures for Obtaining NIST Recognition as an Accreditor (NISTIR 6440)}

This document provides detailed information regarding the National Voluntary Conformity Assessment Systems Evaluation Program (NVCASE) evaluation requirements leading to an organization obtaining NIST recognition as an authorized accreditation body under provisions of certain other agency programs or specified Mutual Recognition Agreements with other countries or geographic areas. NVCASE covers three basic types of accreditors: 1) laboratory; 2) product certifier; and 3) quality system registrar. Order from NTIS by \#PB 2000-101337.

\section{Laboratory Accreditation in the United States (NISTIR 4576)}

This report, a companion to NBSIR 87-3576 The ABC'S of Standards-Related Activities in the United States and NBSIR 883821 The ABC'S of Certification Activities in the United States, is designed to provide information on laboratory accreditation to readers who are new to this field. It discusses some of the more significant facets of this topic, provides information necessary to make informed decisions on the selection and use of laboratories, and serves as background for using other available documents and services. Order from NTIS by \#PB 91-194495.

\section{National Voluntary Laboratory Accreditation Program (NVLAP) Directory (NIST SP 810)}

This annual directory lists laboratories that have been found to be competent to perform certain tests or calibrations as specified. These laboratories are allowed to use the NVLAP logo on their test or calibration certificates or reports, which implies that the processes used to achieve the tests or calibrations have been evaluated by NVLAP as being technically adequate when performed under the conditions specified in the laboratories' quality manuals and associated documentation. Copies are available from NVLAP at (301) 975-4016; fax: (301) 926-2884 or e-mail - nvlap@nist.gov. A listing of accredited laboratories, updated quarterly, is available on the NVLAP website at http://ts.nist.gov/nvlap.

\section{Directory of Federal Government Laboratory Accreditation/Designation Programs $\quad$ (NIST SP 808)}

This directory provides updated information on 31 federal government laboratory accreditation and similar type programs conducted by the federal government. These programs, which include some type of assessment regarding laboratory capability, designate sets of laboratories or other entities to conduct testing to assist federal agencies in carrying out their responsibilities. The directory also lists 13 other federal agency programs of possible interest, including programs involving very limited laboratory assessment and programs still under development. Order from NTIS by \#PB 91-167379. 
Directory of Professional/Trade Organization Laboratory Accreditation/Designation Programs (NIST SP 831, 1999 Edition)

This update is a guide to laboratory accreditation and similar types of programs conducted by professional and trade organizations. These programs accredit or designate laboratories or other entities to assist private sector professional societies, trade associations, related certification bodies, their membership, as well as government agencies, in carrying out their responsibilities. This accreditation or designation is based on an assessment of the capability of the laboratory to conduct the testing. However, the nature of the assessment varies considerably by organization and program. Order from NTIS by \#PB 2000-100398.

Directory of State and Local Government Laboratory Accreditation/Designation Programs (NIST SP 815) This directory provides updated information on 21 state and 11 local government laboratory accreditation and similar type programs. These programs, which include some type of assessment regarding laboratory capability, designate private sector laboratories or other entities to conduct testing to assist state and local government agencies in carrying out their responsibilities. Entries describe the scope and nature of each program, laboratory assessment criteria and procedures used in the program, products and fields of testing covered, program authority, and other relevant details. Order from NTIS by \#PB 92-108968.

\section{A Selective Review of Testing Laboratory Accreditation Movements in the United States (NIST GCR 98-740) \\ In conjunction with a cooperative effort by the Laboratory Accreditation Working Group (LAWG), consisting of public and private sector entities that call for laboratories to be accredited, the affected laboratories, and accreditation bodies, the author has conducted a selective review of a number of previous attempts to systemize laboratory accreditation activities in the United States. In conclusion, a number of recommendations are made for the developing National Cooperation on Laboratory Accreditation (NACLA), the proposed formal structure to succeed the planning organization, LAWG. $\underline{\text { Order }}$ from NTIS by \#PB 99-175606.}

Report on the Open Forum on Establishment of the National Council for Laboratory Accreditation (NACLA) at the National Institute of Standards and Technology January 7, 1997 (NISTIR 6008)

The forum was jointly sponsored by NIST, ACIL (formerly the American Council of Independent Laboratories), and the American National Standards Institute (ANSI). More than 300 representatives from private industry and the government attended it. The purpose of the Forum was to discuss a proposal to establish the National Council for Laboratory Accreditation (NACLA), which would be a cooperative partnership between the public and private sectors designed to provide a national infrastructure for laboratory accreditation in the United States. Order from NTIS by \#PB 97-158133.

Examination of Laboratory Accreditation Programs in the United States and the Potential Role for a National Laboratory Accreditation System (NIST GCR 97-714)

This report presents an initial study of existing U.S. laboratory accreditation programs, with a focus on government programs, particularly at the Federal level. The study was conducted in two phases: Phase I established categories of existing laboratory accreditation programs in the Federal government, at the state and local level, and in the private sector. Phase II compared technical standards used by five Federal government laboratory accreditation programs with general standards for laboratory accreditation established by ISO. Order from NTIS by \#PB 97-167365.

\section{Proceedings of the Open Forum on Laboratory Accreditation at the National Institute of Standards and Technology October 13, 1995 (NIST SP 902) \\ The American National Standards Institute and ACIL requested that NIST work with them in an informal Laboratory Accreditation working group (LAWG) to evaluate the current situation in laboratory accreditation in the United States. This group sponsored a forum to hear reports from various sectors and to arrive at some consensus on the need to improve the current situation and infrastructure for laboratory accreditation in the United States. Order from NTIS by \#PB 96-210141.}




\section{REPORTS ON THE IMPLEMENTATION OF THE NATIONAL TECHNOLOGY TRANSFER AND ADVANCEMENT ACT (NTTAA)}

[Note: Additional information on the NTTAA is available at: http://standards.gov/ and at:

http://ts.nist.gov/ts/htdocs/210/nttaa/nttaa.htm]

\section{The National Technology Transfer and Advancement Act - Plan for Implementation (NISTIR 5967)}

The National Technology Transfer and Advancement Act (P.L. 104-113) gives NIST responsibility to coordinate standards and conformity assessment activities with other Federal agencies, state and local governments, and with the private sector. Congress required NIST to submit a plan for implementing the coordination activities. Specific activities in strategic standards management, responsiveness to international trade concerns, greater use of voluntary standards, and conformity assessment procedures are described. Responsibilities of governments, standards developers, and private sector interests are outlined, as are a number of specific tasks. Order from NTIS by \#PB 97-153001.

\section{Towards Strategic Management of Standards Activities at NIST (NISTIR 6292)}

This paper describes strategic standards management, its use by U.S. companies, and the potential use of this important methodology by DoC/NIST and other government agencies. It also describes the role of NIST's Standards Advisory Committee (SAC) in defining a plan that each NIST Operating Unit (OU) can tailor to manage its own standards activities, and other agencies can similarly adopt. A checklist is provided for use by NIST OUs as they set priorities and implement activities in support of the standards-related work associated with their missions. This checklist can also be adapted for use by other Federal agencies. Order from NTIS by \#PB 99-127896.

\section{Using Voluntary Standards in the Federal Government (NISTIR 6086)}

This report is a compilation of presentations given at a NIST-sponsored conference held on September 8, 1997 to foster better understanding among Federal agencies of the private sector standardization process. The conference took place as part of a major effort by NIST to implement the National Technology Transfer and Advancement Act which gives NIST responsibility to coordinate standards and conformity assessment activities with other Federal agencies, state and local governments, and with the private sector. Order from NTIS by \#PB 98-110281.

\section{Guidelines for NIST Staff Participating in Voluntary Standards Developing Organizations' Activities (NISTIR 6778)}

These guidelines were prepared by the NIST, Standards Services Division (SSD that coordinates the formulation and implementation of the voluntary standards and conformity assessment policies of the federal government. A revision of the October 1989 guidelines, this document provides guidance and background for NIST staff considering or beginning participation in voluntary standards activities and NIST managers who support these activities. Order from NTIS by \#PB 2002-106711).

Annual Report to the Office of Management and Budget on the Implementation of OMB Circular A-119 and P.L. 104-113 This report describes the progress that Federal agencies have made in the use of voluntary consensus standards and participation in standardization activities. It also highlights some examples of agency's interactions with private-sector voluntary consensus standards bodies, agency's use of government unique standards, and the activities of the Interagency Committee on Standards Policy. It discusses the National Institute of Standards and Technology's efforts in coordinating Federal agencies standards-related activities including interactions with key private sector organizations. Copies of the latest and past reports are available at the following web site: $\mathrm{http}$ ://ts.nist.gov/ssd, select 'National Technology Transfer and Advancement Act (NTTAA)', click on 'NTTAA Library'. 


\title{
REPORTS ON INTERNATIONAL STANDARDS AND CONFORMITY ASSESSMENT SYSTEMS
}

\author{
A Review of U.S. Participation in the International Organization for Standardization (ISO) and the International \\ Electrotechnical Commission (IEC) (NISTIR 6492) \\ This report describes the role of international standards, their importance in world trade, and the extent of U.S. participation \\ in the International Organization for Standardization (ISO) and the International Electrotechnical Commission (IEC) over the \\ 32-year period from 1966 to 1998. To the extent possible, a comparison of U.S. exports with U.S. participation in the ISO \\ and IEC is provided. Mention is also made of the World Trade Organization's Agreement on Technical Barriers to Trade. \\ Order from NTIS by \#PB 2000-105984.
}

Directory of International and Regional Organizations Conducting Standards-Related Activities (NIST SP 767) This directory contains information on 338 international and regional organizations, which conduct standardization, certification, laboratory accreditation, or other standards-related activities. It describes their work in these areas, as well as the scope of each organization, national affiliations of members, U.S. participants, restrictions on membership, and the availability of any standards in English. Order from NTIS by \#PB 89-221147 or Global Engineering Documents by Order \#Cat.SP767.

\section{ISO Environmental Management Standardization Efforts (NISTIR 5638-1)}

This report describes the development of planned "environmental management" standards by the International Organization for Standardization (ISO). These standards address management systems and the environmental aspects of products in the areas of life cycle assessment and labeling. The report outlines the current status of the ISO standards and also covers developments relating to third party certification of environmental management systems. Order from NTIS by \#PB 96158662. Updated information on this topic is also available on the following website: http://ts.nist.gov/ts/htdocs/210/gsig/iso14.htm

\section{Questions and Answers on Quality, the ISO 9000 Standard Series, Quality System Registration, and Related Issues} (NISTIR 4721)

This report provides information on the development, content and application of the ISO 9000 standards to readers who are unfamiliar with these aspects of the standards. It attempts to answer some of the most commonly asked questions on quality; quality systems; the content, application and revision of the ISO 9000 standards; quality system approval/registration; European Community requirements for quality system approval/registration; and sources for additional help. Order from NTIS by \#PB 93-152080/AS. Updated information on this topic is also available on the following website: http://ts.nist.gov/ts/htdocs/210/gsig/iso9000.htm

\section{More Questions and Answers on the ISO 9000 Standard Series and Related Issues (NISTIR 5122)}

This report, a sequel to NISTIR 4721, provides additional information on the ISO 9000 standards and related issues to readers unfamiliar with some of the new developments in this area. It attempts to answer additional questions on ISO 9000 standards related issues which NIST has received since the publication of NISTIR 4721 and identifies sources for further help in this area. Order from NTIS by \#PB 93-140689. Updated information on this topic is also available on the following website: http://ts.nist.gov/ts/htdocs/210/gsig/iso9000.htm 


\title{
REPORTS ON REGIONAL STANDARDS AND CONFORMITY ASSESSMENT SYSTEMS - EUROPEAN UNION
}

\author{
A Guide to EU Standards and Conformity Assessment (NIST SP 951) \\ This guide is an easy-to-use introductory reference for industry and government officials on the general principles and \\ concepts behind the European Union's (EU) "New Approach" laws and directives. It is designed to help business and \\ government officials understand the new laws, the EU's standardization process, and the relationship between the European \\ Commission and the European standardization bodies in the European Union. Order from NTIS by \#PB 2000-105983. \\ Also available at the following web site: http://ts.nist.gov/ncsci, click on 'European Union.'
}

\section{A Guide to the EU In vitro Diagnostic Medical Devices Directive (NIST GCR 01-817)}

This guide is an easy-to-use introductory reference for industry and government officials on the requirements of the European Union's (EU) In vitro Diagnostic Medical Device Directive [Directive 98/79/EEC]. Order from NTIS by \#PB 2001$\underline{106308 .}$

\section{A Guide to the EU Active Implantable Medical Devices Directive (NIST GCR 01-816)}

This guide is an easy-to-use introductory reference for industry and government officials on the requirements of the European Union's (EU) Active Implantable Medical Device Directive [Directive 90/385/EEC]. Order from NTIS by \# PB 2001106309.

\section{A Guide to the EU Medical Device Directive (NIST GCR 01-815)}

This guide is an easy-to-use introductory reference for industry and government officials on the requirements of the European Union's (EU) Medical Device Directive (MDD) (93/42/ECC). Order from NTIS by \#PB 2001-106759.

\section{A Guide to the EU Machinery Directive (NIST GCR 01-814)}

This guide is an easy-to-use introductory reference for industry and government officials on the requirements of the European Union's (EU) Machinery Directive [Directive 98/37/EEC]. Order from NTIS by \#PB 2001-105514.

A Guide to the EU Directive on Electromagnetic Compatibility (NIST GCR 01-813)

This guide is an easy-to-use introductory reference for industry and government officials on the requirements of the European Union's (EU) Electromagnetic Compatibility (EMC) Directive [Directive 89/336/EEC]. Order from NTIS by \#PB 2001$\underline{105513 .}$

A Guide to the EU Low Voltage Directive (NIST GCR 01-812)

This guide is an easy-to-use introductory reference for industry and government officials on the requirements of the European Union's (EU) Low Voltage Directive (LVD) [Directive 73/23/EEC]. Order from NTIS by \#PB 2001-107673.

\section{A Guide to the EU Safety of Toys Directive (NIST GCR 01-823)}

This report is an easy-to-use introductory reference for industry and government officials on the requirements of the European Union's (EU) Safety of Toys Directive [88/378/EEC]. It is designed to help business and government officials understand the purpose of the directive, its relationship to other directives, the essential requirements contained in the directive, and the basic steps necessary for compliance. The guide offers explanations of such requirements as: the products covered by the directive; particular risks associated with toys and specific guidelines for those risks; toy hygiene; radioactivity toys; warnings and indications of precautions; and the use of standards. The guide contains the text of the directive and a list of applicable standards. In addition, the guide references appropriate sections of NIST Special Publication 
951: A Guide to EU Standards and Conformity Assessment for further information on some of the generic conformity assessment concepts and requirements of the EU's New Approach. Order from NTIS by \#PB 2001-109030.

A Guide to the EU Directive Concerning Liability for Defective Products (Product Liability Directive) (NIST GCR 01-824) This guide is an easy-to-use introductory reference for industry and government officials on the requirements of the European Union's (EU) Directive Concerning Liability for Defective Products (Product Liability Directive) [85/374/ECC]. Order from NTIS by \#PB 2001-109029.

A Guide to the EU Directive on General Product Safety (NIST GCR 01-825)

This guide is an easy-to-use introductory reference for industry and government officials on the requirements of the European Union's (EU) Directive on General Product Safety [92/59/EEC]. Order from NTIS by \#PB 2002-100126.

A Guide to the EU Directive on Radio Equipment and Telecommunications Terminal Equipment (NIST GCR 01-826) This guide is an easy-to-use introductory reference for industry and government officials on the requirements of the European Union's (EU) Directive on Radio Equipment and telecommunications Terminal Equipment (1999/5/EC). Order from NTIS by \#PB 2002-100125.

Standards Setting in the European Union - Standards Organizations and Officials in EU Standards Activities (NIST SP 891, 1997 Edition)

The guide is designed to help U.S. manufacturers, exporters, and other interested persons in locating contact points for important information on the development of standards and conformity assessment issues. The report includes a history of the role of standards in the European Union (EU) and the latest information on the EU's harmonization directives for implementing the "New Approach" and the "Global Approach" for harmonizing technical regulations and standards to reduce barriers to trade. Order from NTIS as \#PB 97-153738.

\section{Directory of European Regional Standards-Related Organizations (NIST SP 795)}

This directory identifies more than 150 European regional organizations - both governmental and private - that engage in standards development, certification, laboratory accreditation and other standards-related activities, such as quality assurance. Entries describe the type and purpose of each organization; acronyms; national affiliations of members; the nature of the standards-related activity; and other related information. Order from NTIS by \#PB 91-107599 or Global Engineering Documents by Order \#Cat. 0258-3.

\section{REPORTS ON REGIONAL STANDARDS AND CONFORMITY ASSESSMENT SYSTEMS - ASIA-PACIFIC ECONOMIC COOPERATION (APEC)}

An Overview of the Development of Technical Infrastructure in the Asia-Pacific Region: The Work of the Asia-Pacific Economic Cooperation (APEC) Sub-committee on Standards and Conformance (SCSC) and the Specialist Regional Bodies (SRBS) (NISTIR 6325)

The development and maintenance of an economy's standards and conformance technical infrastructure is critical to its economic health. By increasing the competence of its measurement and testing capabilities, in particular, an economy can provide substantial benefits to its manufacturers and consumers. It is also better equipped to participate in the confidence building requirements of the region to take advantage of increased trade opportunities. Technical infrastructure development is a major focus of the Asia-Pacific Economic Cooperation Sub-committee on Standards and Conformance and the Specialist Regional Bodies, which work together closely to develop practical programs to assist this development. Order from NTIS by \#PB 99-142580. 


\title{
REPORTS ON REGIONAL STANDARDS AND CONFORMITY ASSESSMENT SYSTEMS - THE AMERICAS
}

\author{
Free Trade Area of the Americas (FTAA) Conformity Assessment Infrastructure \\ (NIST SP 941) \\ This publication summarizes the status of the technical infrastructure for conformity assessment in the Americas. It provides \\ information about inspection and testing, product certification, quality system registration and laboratory accreditation for the \\ countries that will comprise the Free Trade Area of the Americas. Order from NTIS by \#PB 99-167447.
}

\author{
****Directory of Accredited North American Quality System Registration Organizations (NAQSRO) (NISTIR 6515, 2002 \\ Edition) \\ This semi-annual listing provides information on organizations that have informed NIST about their quality system \\ registration according to the ASQ Q-9000 series, QS-9000, ISO 14000 (EMS) or equivalent criteria for the United States, \\ Canada and Mexico. Order from NTIS by PB2003-100541 (NISTIR 65152002 ED) or \\ Copies are available from the Standards Coordination and Conformity Group at (301) 975-4039, fax: (301) 975-5414; \\ e-mail - sccg@nist.gov. It is also available at the following web site: http://ts.nist.gov/gsig, go to 'Related \\ Information,' and click on 'ISO 9000 information.'
}

\section{REPORTS ON FOREIGN NATIONAL STANDARDS AND CONFORMITY ASSESSMENT SYSTEMS}

\section{Profiles of National Standards-Related Activities (NIST SP 912)}

This directory describes the metrology, standardization, testing and quality (MSTQ) activities of more than 70 countries. Each entry includes basic data on the country's economy and trade; agencies and institutions responsible for metrology and calibration, standards development, testing, product certification, quality and environmental system registration and accreditation; and key contacts and information sources. Entries are formatted to facilitate access to specific information. An introductory section provides general information on development of the directory and an overview of world-wise MSTQ activities. Order from NTIS by \#PB 97-169874/AS.

Survev on the Implementation of ISO/IEC Guide 25 bv National Laboratorv Accreditation Programs (NISTIR 5473) ISO/IEC Guide 25, General Requirements for the Competence of Calibration and Testing Laboratories, has been used by many laboratory accreditation programs worldwide to establish accreditation requirements designed to promote confidence in the calibrations and testing results of laboratories. National delegations to the International Laboratory Accreditation Conference (ILAC) were surveyed to collect information on the implementation and supplementation of the requirements of ISO/IEC Guide 25 within the context of their countries' laboratory accreditation programs. This report summarizes the results of that survey and includes a bibliographic list of publications concerned with ISO/IEC Guide 25 implementation complied from the information by the national delegations. Order from NTIS by \#PB 94-210150. 


\title{
TO ORDER PUBLICATIONS
}

\section{NOTE: Many of these publications are available via the Internet at http://ts. nist.gov/ncsci or http://ts.nist.gov/ssd.}

\author{
National Technical Information Service (NTIS) \\ 285 Port Royal Road \\ Springfield, Virginia 22161, USA \\ Telephone: (703) 605-6000 \\ Orders Only: (800) 553-6847 \\ Fax: (703) 605-6900 \\ Email: orders@ntis.gov \\ Internet: http://www.ntis.gov
}

\author{
Global Engineering Documents \\ 15 Inverness Way East \\ Englewood, Colorado 80112-5704 USA \\ Telephone: (800) 854-7179 or (303) 397-7956 \\ Fax: (303) 397-2740 \\ Email: global@ihs.com \\ Internet: http://global.ihs.com
}




\begin{abstract}
APPENDIX IV
OTHER LISTS OF STATE LABORATORY ACCREDITATION/DESIGNATION BODIES OR RELATED ORGANIZATIONS
\end{abstract}

- LIST OF STATE NELAP RECOGNIZED ACCREDITING AUTHORITIES. .87

- LIST OF CLIA STATE SURVEY AGENCIES .......................................89

- LIST OF STATE GRADE 'A' MILK SANITATION PERSONNEL ...................... 97

- LIST OF STATE CERTIFICATION OFFICERS FOR DRINKING WATER

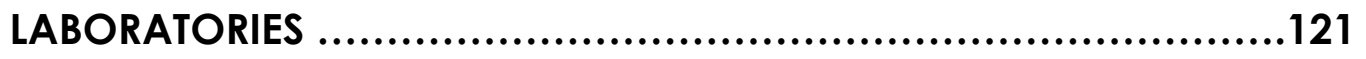

- LIST OF STATE CONTACT INFORMATION AVAILABLE FROM FEDERAL AGENCIES ......................................................... 129

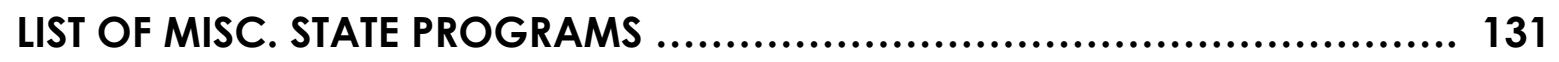




\section{LIST OF STATE NELAP RECOGNIZED ACCREDITING AUTHORITIES Available at: http://www.epa.gov/ttn/nelac/nelapaa.html}

\begin{tabular}{|c|c|c|c|c|}
\hline CALIFORNIA & $\begin{array}{l}\text { Environmental } \\
\text { Laboratory } \\
\text { Accreditation } \\
\text { Program }\end{array}$ & $\begin{array}{l}\text { Dr. George } \\
\text { Kulasingam }\end{array}$ & $\begin{array}{l}\text { T:510/540-2800 } \\
\text { F:510/849-5106 } \\
\text { E: gkulasin@dhs.ca.gov }\end{array}$ & $\begin{array}{l}\text { www.dhs.ca.gov/ps/ls/elap/elapindex.htm } \\
\text { caaainfo.pdf } \operatorname{PDF}(2.6 \mathrm{~K})\end{array}$ \\
\hline FLORIDA & $\begin{array}{l}\text { FL Dept of Health, } \\
\text { Bureau of } \\
\text { Laboratories }\end{array}$ & Mr. Stephen Arms & \begin{tabular}{|l|} 
T:904/791-1502 \\
F:904/791-1591 \\
E: steve_arms@doh.state.fl.us
\end{tabular} & www.floridadep.org/labs/qa/dohforms.htm \\
\hline ILLINOIS & $\begin{array}{l}\text { EPA, Div. of Lab., } \\
\text { QA Section }\end{array}$ & Mr. Jim Shaw & $\begin{array}{l}\text { T:217/782-6455 } \\
\text { F:217/524-0944 } \\
\text { E: epa6109@epa.state.il.us }\end{array}$ & www.epa.state.il.us/labs/index.html \\
\hline KANSAS & $\begin{array}{l}\text { KS Dept. of Health } \\
\& \text { Environment }\end{array}$ & Mr. Stan Sutton & $\begin{array}{l}\text { T:785/296-1640 } \\
\text { F:785/296-1641 } \\
\text { E: ssutton@kdhe.state.ks.us }\end{array}$ & www.kdhe.state.ks.us/lipo/index.html \\
\hline LOUISIANA & $\begin{array}{l}\text { LA Dept. of Health } \\
\& \text { Hospitals }\end{array}$ & Mr. Louis Wales & $\begin{array}{l}\text { T:504/568-5359 } \\
\text { F:504/568-8057 } \\
\text { E:1wales@dhh.state.la.us }\end{array}$ & Not Available \\
\hline LOUISIANA & $\begin{array}{l}\text { LA Dept. of } \\
\text { Environmental } \\
\text { Quality }\end{array}$ & $\begin{array}{l}\text { Mr. Louis R. C. } \\
\text { Johnson }\end{array}$ & $\begin{array}{l}\text { T:225/765-0582 } \\
\text { F:225/765-2408 } \\
\text { E:louis_j@deq.state.la.us }\end{array}$ & www.deq.state.la.us \\
\hline NEW HAMPSHIRE & $\begin{array}{l}\text { NH Environmental } \\
\text { Lab. Accreditation } \\
\text { Program }\end{array}$ & Mr. Charles Dyer & $\begin{array}{l}\text { T:603/271-2991 } \\
\text { F:603/271-2997 } \\
\text { E: c_dyer@des.state.nh.us }\end{array}$ & www.des.state.nh.us/nhelap \\
\hline NEW JERSEY & \begin{tabular}{|l} 
Dept. of \\
Environmental \\
Protection
\end{tabular} & Mr. Joseph Aiello & $\begin{array}{l}\text { T:609/292-3950 } \\
\text { F:609/777-1774 } \\
\text { E: jaiello@dep.state.nj.us }\end{array}$ & njaainfo.pdf $\underline{\text { PDF }}$ \\
\hline NEW YORK & $\begin{array}{l}\text { NY State Dept. of } \\
\text { Health }\end{array}$ & $\begin{array}{l}\text { Dr. Kenneth } \\
\text { Jackson }\end{array}$ & $\begin{array}{l}\text { T:518/485-5570 } \\
\text { F:518/485-5568 } \\
\text { E: jackson@wadsworth.org }\end{array}$ & www.wadsworth.org/labcert \\
\hline OREGON & $\begin{array}{l}\text { Oregon Health } \\
\text { Division }\end{array}$ & $\begin{array}{l}\text { Dr. Irene E. } \\
\text { Ronning }\end{array}$ & \begin{tabular}{|l|}
$\mathrm{T}: 503 / 229 / 5505$ \\
F:503/229-5682 \\
E: irene.e.ronning@state.or.us
\end{tabular} & www.deq.state.or.us/lab/ORELAP/orelap.htm \\
\hline PENNSYLVANIA & $\begin{array}{l}\text { Bureau of Labs, } \\
\text { Dept. of Environ. } \\
\text { Prot. }\end{array}$ & $\begin{array}{l}\text { Mr. Richard } \\
\text { Sheibley }\end{array}$ & $\begin{array}{l}\text { T:717/783-7150 } \\
\text { F:717/783-1502 } \\
\text { E: rsheibley@state.pa.us }\end{array}$ & www.dep.state.pa.us/dep/deputate/mts/bol \\
\hline UTAH & Utah Dept. of Health & $\begin{array}{l}\text { Mr. Dave } \\
\text { Mendenhall }\end{array}$ & $\begin{array}{l}\text { T:801/584-8470 } \\
\text { F:801/584-8501 } \\
\text { E: dmendenh@doh.state.ut.us }\end{array}$ & hlunix.hl.state.ut.us/els/lamimp \\
\hline
\end{tabular}




\title{
LIST OF \\ CLIA STATE SURVEY AGENCIES \\ CONTACT LIST
}

(The Clinical Laboratory Improvement Amendments of 1988 (CLIA) program ensures quality laboratory testing by requiring that all clinical laboratories must be properly certified to receive Medicare or Medicaid payments)

(Available at: http://cms.hhs.gov/clia/saaddres.asp\#al)

\author{
$\underline{\text { REGION I - Boston }}$ \\ CLIA LABORATORY PROGRAM \\ DEPARTMENT OF PUBLIC HEALTH \\ 410 Capitol Avenue, MS\#12HSR \\ PO BOX 340308 \\ HARTFORD, CT 06134-0308 \\ (860) 509-7400 \\ FAX: (860) 509-7543 \\ DEPARTMENT OF PUBLIC HEALTH \\ CLINICAL LABORATORY PROGRAM \\ 305 SOUTH STREET, RM. 224 \\ JAMAICA PLAIN, MA 02130 \\ (617) 983-6739 \\ FAX: (617) 983-6740 \\ CLIA LABORATORY PROGRAM \\ DIVISION OF FACILITIES REGULATION \\ DEPARTMENT OF HEALTH \\ 3 CAPITOL HILL \\ PROVIDENCE RI 02908 \\ (401) 222-4526 \\ FAX: (401) 222-3999 \\ CLIA LABORATORY PROGRAM \\ DIVISION OF LICENSING \& CERTIFICATION \\ 442 Civic Center Drive, Station \#11 \\ AUGUSTA ME 04330 \\ (207) 287-9339 \\ FAX: (207) 287-9304 \\ CLIA LABORATORY PROGRAM \\ HEALTH FACILITIES ADMINISTRATION \\ DEPARTMENT OF HEALTH \& HUMAN SERVICES \\ 129 PLEASANT STREET \\ CONCORD, NH 03301 \\ (603) 271-4832 \\ FAX: (603) 271-4968 \\ VERMONT DEPARTMENT OF HEALTH \\ CLIA LABORATORY PROGRAM
}


108 CHERRY STREET

BURLINGTON, VT 05402-1125

(802) 652-4145

FAX: (802) 865-7701

\section{REGION II - New York}

CLINICAL LABORATORY IMPROVEMENT SERVICE

STATE OF NEW JERSEY

DEPARTMENT OF HEALTH \& SENIOR SERVICES

CN 360

TRENTON, NJ 08625-0360

(609) 292-0016

FAX: (609) 292-9285

STATE OF NEW YORK DEPARTMENT OF HEALTH

PHYSICIAN OFFICE LABORATORY EVALUATION PROGRAM

EMPIRE STATE PLAZA

PO BOX 509

ALBANY, NY 12201-0509

(518) 485-5352

COMMONWEALTH OF PUERTO RICO

PUERTO RICO HEALTH DEPARTMENT

OFFICE OF CERTIFICATION \& LICENSURE

FORMER-RUIZ SOLER HOSPITAL

ROAD NO. 2

BAYAMON, PR 00619

(787) 782-0120 Ext. 2222

$* * * * *$ FOR LABS IN VIRGIN ISLANDS (48) DIRECT THEM TO NEW YORK RO (33)**** REGION III - Philadelphia

OFFICE OF HEALTH FACILITIES

LICENSING AND CERTIFICATION

2055 LIMESTONE ROAD, SUITE 200

WILMINGTON, DE 19808

(302) 653-2870

DEPARTMENT OF CONSUMER \& REGULATORY AFFAIRS

825 N. CAPITAL STREET NE, 2nd Fl.

WASHINGTON, DC 20002

(202) 442-4706

MARYLAND DEPARTMENT OF HEALTH \& MENTAL HYGIENE

OFFICE OF HEALTH CARE - LABS

BLAND BRYANT BUILDING

SPRING GROVE HOSPITAL CENTER

55 WADE AVE.

CATONSVILLE, MD 21228

(410) 402-8025

PENNSYLVANIA DEPARTMENT OF HEALTH

BUREAU OF LABORATORIES

PO BOX 500

EXTON, PA 19341-0500 
(610) $280-3464$

VIRGINIA DEPARTMENT OF HEALTH

CENTER FOR QUALITY HEALTH CARE SERVICES

$3600 \mathrm{~W}$ BROAD ST, Suite 216

RICHMOND, VA 23230

(804) 367-2107

WEST VIRGINIA DEPARTMENT OF HEALTH

OFFICE OF LABORATORY SERVICES

167 11TH AVENUE

SOUTH CHARLESTON, WV 25303-1137

(304) 558-3530

\section{REGION IV - Atlanta}

ALABAMA DEPARTMENT OF PUBLIC HEALTH

DIVISION OF HEALTH CARE FACILITIES

CLIA PROGRAM,

P.O. BOX 303017

MONTGOMERY, AL 36130-3017

(334) 206-5120

STATE OF FLORIDA

AGENCY FOR HEALTH CARE ADMINISTRATION

LABORATORY LICENSING UNIT

2727 MAHAN DR, mail Stop 32

TALLAHASSEE, FL 32308

(850) 487-3109

Fax - (850) 410-1511

GEORGIA DEPARTMENT OF HUMAN RESOURCES

OFFICE OF REGULATORY SERVICES

DIAGNOSTIC SERVICE UNIT

2 PEACHTREE STREET, N.W. 33-250

ATLANTA, GA 30303-3142

(404) 657-5447

DIVISION OF LICENSING \& REGULATION

CABINET FOR HEALTH SERVICES

275 E MAIN ST

FRANKFORT, KY 40621

(502) 564-2800

LICENSURE AND CERTIFICATION

MISSISSIPPI DEPARTMENT OF PUBLIC HEALTH

PO BOX 1700

JACKSON, MS 39215-1700

(601) $576-7300$

NORTH CAROLINA DEPARTMENT OF HUMAN SERVICES DIVISION OF FACILITY SERVICES/CLIA CERTIFICATION 2713 MAIL SERVICE CENTER

RALEIGH, NC 27699-2713

(919) $733-1610$

Fax - (919) 733-0176 
SOUTH CAROLINA DEPARTMENT OF HEALTH \& ENVIRONMENTAL CONTROL BUREAU OF HEALTH LICENSURE \& CERTIFICATION

2600 BULL ST

COLUMBIA, SC 29201

(803) 545-4203

TENNESSEE HEALTH CARE FACILITIES

CORDELL HULL BLDG, 1st FL

425 5TH AVENUE NORTH

NASHVILLE, TN 37247-0508

(615) 741-7023

\section{REGION V - Chicago}

ILLINOIS DEPARTMENT OF PUBLIC HEALTH

DIVISION OF HEALTH CARE FACILITIES \& PROGRAMS

525 W JEFFERSON ST

FOURTH FLR

SPRINGFIELD, IL 62761

(217) 782-7412

INDIANA STATE DEPARTMENT OF HEALTH

DIVISION OF ACUTE CARE SERVICES

2 NORTH MERIDIAN ST

INDIANAPOLIS, IN 46204

(317) 233-7502

MICHIGAN DEPARTMENT OF CONSUMER \& INDUSTRY SERVICES

LABORATORY IMPROVEMENT SECTION

BOX 30664

LANSING, MI 48909

(517) 241-2648

MINNESOTA DEPARTMENT OF HEALTH

LICENSING \& CERTIFICATION SECTION

85 EAST SEVENTH PLACE, SUITE 300

BOX 64900

ST PAUL, MN 55164-0900

(651) 215-8704

OHIO DEPARTMENT OF HEALTH

LABORATORY CERTIFICATION PROGRAM

246 N HIGH ST FIFTH FLR

COLUMBUS, OH 43266-0588

(614) 644-1845

WISCONSIN DEPARTMENT OF HEALTH \& FAMILY SERVICES

DIVISION OF SUPPORTIVE LIVING

CLINICAL LABORATORY UNIT/BQA

PO BOX 2969

MADISON, WI 53701-2969

(608) 266-5753 


\section{$\underline{\text { REGION VI - Dallas }}$}

DIVISION OF HEALTH FACILITY SERVICES

ARKANSAS DEPARTMENT OF HEALTH

$5800 \mathrm{~W} 10$ th ST STE 400

LITTLE ROCK, AR 72204

(501) 661-2201

DEPARTMENT OF HEALTH \& HOSPITALS

HEALTH STANDARDS SECTION

655 N. 5TH. STREET

BATON ROUGE, LA 70821

(225) 342-9324

DEPARTMENT OF HEALTH

DIVISION OF HEALTH IMPROVEMENT

HEALTH FACILITY LICENSING AND CERTIFICATION BUREAU

CLIA PROGRAM

2040 South Pacheco Street 2nd Floor, Room 413

SANTA FE, NM 87505

(505) $841-4524$

Fax: (505) 841-5834

OKLAHOMA STATE DEPARTMENT OF HEALTH

SPECIAL HEALTH SERVICES

MEDICAL FACILITIES

1000 NE 10TH STREET

OKLAHOMA CITY, OK 73117-1299

(405) 271-6576

HEALTH FACILITY COMPLIANCE DIVISION

TEXAS DEPARTMENT OF HEALTH

1100 WEST 49TH STREET

AUSTIN, TX 78756-3199

(512) $834-6650$

\section{REGION VII - Kansas City}

IOWA DEPARTMENT OF INSPECTIONS \& APPEALS

DIVISION OF HEALTH FACILITIES

LUCAS STATE OFFICE BUILDING 3RD FL

DES MOINES, IA 50319-0083

(515) 281-3765

KANSAS DEPARTMENT OF HEALTH \& ENVIRONMENT

LABORATORY CERTIFICATION

BUILDING 740

FORBES FIELD

TOPEKA, KS 66620-0001

(785) 296-3811

MISSOURI DEPARTMENT OF HEALTH

CLIA SECTION

PO BOX 570

JEFFERSON CITY, MO 65102 
(573) $751-6318$

NEBRASKA STATE HEALTH \& HUMAN SERVICES

REGULATIONS AND LICENSURE

CREDENTIALING DIVISION

PO BOX 95007

LINCOLN, NE 68509-5007

(402) 471-4363

\section{REGION VIII - Denver}

COLORADO DEPARTMENT OF PUBLIC HEALTH \& ENVIRONMENT

CERTIFICATION PROGRAMS

LABORATORY \& RADIATION SERVICES DIVISION

8100 Lowry Blvd.

DENVER, CO 80230-6928

(303) 692-3295

FAX: (303) 344-9965

CERTIFICATION BUREAU

DEPARTMENT OF PUBLIC HEALTH \& HUMAN SERVICES

COGSWELL BUILDING

PO BOX 202951

HELENA, MT 59620-2951

(406) 444-1451

FAX: (406) 444-3456

HEALTH RESOURCES SECTION

NORTH DAKOTA DEPARTMENT OF HEALTH

STATE CAPITAL

600 EAST BOULEVARD AVENUE/DEPT 301

BISMARCK, ND 58505-0200

(701) 328-2352

FAX: (701) 328-4727

SOUTH DAKOTA DEPARTMENT OF HEALTH LICENSURE AND CERTIFICATION 615 E 4TH STREET

PIERRE, SD 57501-1700

(605) 773-3694

FAX: (605) 773-6667

UTAH DEPARTMENT OF HEALTH

BUREAU OF LABORATORY IMPROVEMENT

46 NORTH MEDICAL DRIVE

SALT LAKE CITY, UT 84113-1105

(801) 584-8471

FAX: (801) 584-8501

WYOMING DEPARTMENT OF HEALTH

LABORATORY LICENSURE \& CERTIFICATION

2020 CAREY AVE, 8TH FLOOR

CHEYENNE, WY 82002-0480

(307) 777-6057

FAX: (307) 777-5970 


\section{REGION IX - San Francisco}

ARIZONA DEPARTMENT OF HEALTH

BUREAU OF STATE LABORATORY SERVICES

OFFICE OF LABORATORY LICENSURE, CERTIFICATION \& TRAINING

1740 W. ADAMS, ROOM 203 North

PHOENIX, AZ 85007

(602) 364-0741

FAX: (602) 364-0759

CALIFORNIA DEPARTMENT OF HEALTH SERVICES

LABORATORY FIELD SERVICES

DIVISION OF LABORATORY SCIENCE

865 S. FIGUEROA STREET, Suite 360

LOS ANGELES, CA 90017

(213) 833-6000

FAX: (213) 833-6014

HAWAII DEPARTMENT OF HEALTH

CLIA PROGRAM

2725 WAIMANO HOME ROAD

PEARL CITY, HI 96782

(808) 453-6692

FAX: (808) 453-6662

FOR LABS IN GUAM (65) AMERICAN SAMOA (64) OR SAIPAN (66) DIRECT THEM TO HAWAII (12)

NEVADA STATE HEALTH DIVISION

BUREAU OF LIC. AND CERTIFICATION

1550 E. COLLEGE PARKWAY, \#158

CARSON CITY, NV 89706

(775) $687-4475$

FAX: (775) 687-6588

\section{$\underline{\text { REGION X - Seattle }}$}

OFF. OF LABORATORY QUALITY ASSURANCE

DEPARTMENT OF HEALTH

1610 NE 150TH STREET K17-9

SEATTLE, WA 98155-9701

(206) 361-2805

FAX: (206) 361-2813

OREGON STATE PUBLIC HEALTH LABORATORIES

CLIA PROGRAM

PO BOX 275

PORTLAND, OR 97207-0275

(503) 229-5853

FAX: (503) 229-5682

LABORATORY IMPROVEMENT SECTION

BUREAU OF LABORATORIES

2220 OLD PENITENTIARY ROAD

BOISE, ID 83712-8299

(208) $334-2235 \times 245$

FAX: (208) 334-2382 
OFFICE OF HEALTH FACILITIES \& LICENSURE

DEPT. OF HEALTH AND SOCIAL SERVICES

4730 BUSINESS PARK BOULEVARD, SUITE 18

ANCHORAGE, AK 99503-7137

(907) 334-2491

FAX: (907) 561-3011 


\section{LIST OF}

\section{STATE GRADE 'A' MILK SANITATION PERSONNEL}

\section{ALABAMA}

\section{Regulatory}

Alabama Dept. of Health

The RSA Tower

Bur. of Envm. Services

Suite 1250

PO Box 303017

Montgomery, AL 36130-3017

(334) 206-5375

(FAX) 206-5788

\section{Laboratory}

Birmingham Div. of Lab.

AL Dept. of Public Health

PO Box 2646

Birmingham, AL 35233-2646

(205) 933-1388

(FAX) 933-2922

\section{ALASKA}

\section{Regulatory}

Div. Of Envm. Health

Dept. Of Environmental Conservation

500 S. Alaska Street

Palmer, AK 99645

(907) 745-3236

(FAX) 745-8125

\section{Laboratory}

Div. Of Environmental Health

Dept. Of Environmental Conservation

500 S. Alaska Street

Palmer, AK 99645

(907) 745-3236

(FAX) 745-8125

\section{ARIZONA}




\begin{tabular}{|c|}
\hline Regulatory \\
\hline $\begin{array}{l}\text { Arizona Dept. Of Agriculture } \\
\text { Animal Services Div. } \\
\text { Dairy \& Dairy Products Control } \\
\text { 1688 West Adams Street } \\
\text { Phoenix, AZ } 85007 \\
\text { (602) 542-4189 } \\
\text { (FAX) 542-4194 }\end{array}$ \\
\hline Laboratory \\
\hline $\begin{array}{l}\text { AZ Dept. Of Agriculture } \\
2422 \text { W. Holly Street } \\
\text { Phoenix, AZ } 85009 \\
\text { (602) 744-4920 } \\
\text { (FAX) 253-2247 }\end{array}$ \\
\hline ARKANSAS \\
\hline Regulatory \\
\hline $\begin{array}{l}\text { Milk \& Dairy Products Control } \\
\text { AR Dept. Of Health } \\
4815 \text { W. Markham Street, Slot } 46 \\
\text { Little Rock, AR 72205-3867 } \\
\text { (501) 661-2171 } \\
\text { (FAX) 661-2572 }\end{array}$ \\
\hline Laboratory \\
\hline $\begin{array}{l}\text { Microbiology Branch } \\
\text { Div. Of Public Health Labs. } \\
\text { AR Dept. Of Health } \\
\text { 4815 W. Markham Street } \\
\text { Little Rock, AR 72205-3867 } \\
\text { (501) 661-2738 } \\
\text { (FAX) 661-2310 } \\
\end{array}$ \\
\hline CALIFORNIA \\
\hline Regulatory \\
\hline $\begin{array}{l}\text { Milk \& Dairy Foods Control Branch } \\
\text { Div. Of Animal Industry } \\
\text { CA Dept. Of Food \& Agriculture } \\
1220 \text { N Street } \\
\text { Sacramento, CA } 95814 \\
\text { (916) 654-0773 } \\
\text { (FAX) 654-1274 }\end{array}$ \\
\hline
\end{tabular}




\begin{tabular}{|c|}
\hline Laboratory \\
\hline COLORADO \\
\hline Regulatory \\
\hline $\begin{array}{l}\text { CO Dept. Of Public Health \& Environment } \\
\text { Consumer Protection Division } \\
\text { Milk and Dairy Program } \\
4300 \text { E. Cherry Creek Drive South, CPD-MD B-2 } \\
\text { Denver, CO 80246-1530 } \\
\text { (303) 692-3643 } \\
\text { (FAX) 753-6809 }\end{array}$ \\
\hline $\begin{array}{c}\text { Laboratory \& Radiation Services } \\
\text { CO Dept. Of Public Health \& Environment } \\
\text { 8100 Lowry Blvd. } \\
\text { Denver, CO } 80220 \\
\text { (303) 692-3297 } \\
\text { (FAX) 344-9989 }\end{array}$ \\
\hline CONNECTICUT \\
\hline Regulatory \\
\hline $\begin{array}{l}\text { Bur. Of Regulation \& Insp. } \\
\text { State Dept. Of Agriculture } \\
765 \text { Asylum Ave. } \\
\text { Hartford, CT } 06105 \\
\text { (860) 713-2508 } \\
\text { (FAX) 713-2515 }\end{array}$ \\
\hline $\begin{array}{l}\text { Bur. Of Laboratory } \\
\text { CT Dept. Of Health } \\
10 \text { Clinton Street } \\
\text { Hartford, CT 06106-1684 } \\
\text { (860) 509-8500 } \\
\text { (FAX) 509-8691 }\end{array}$ \\
\hline $\begin{array}{l}\text { Environmental Health Div. } \\
\text { CT Dept. Of Public Health } \\
\text { Mall Stop 51 LAB } \\
\text { 450 Capitol Avenue } \\
\text { P.O. Box 340308 } \\
\text { Hartford, CT 06134-0308 } \\
\quad \text { (860) 509-7386 }\end{array}$ \\
\hline
\end{tabular}




\begin{tabular}{|c|}
\hline (FAX) 509-7378 \\
\hline DELAWARE \\
\hline Regulatory \\
\hline $\begin{array}{c}\text { Dairy Safety Program } \\
\text { DE Div. Of Public Health } \\
\text { Jesse Cooper Bldg. } \\
\text { P.O. Box 637 } \\
\text { Dover, DE } 19903 \\
\text { (302) 739-4731 } \\
\text { (FAX) 739-6617 } \\
\end{array}$ \\
\hline Laboratory \\
\hline $\begin{array}{c}\text { Div. Of Laboratories } \\
\text { Div. Of Public Health \& Lab. } \\
\text { DE Dept. Of Health \& Social Services } \\
\text { 30 Sunny Side Road } \\
\text { P.O. Box 1047 } \\
\text { Smyrna, DE 19977-1047 } \\
\text { (302) 653-2870 } \\
\text { (FAX) 653-2877 }\end{array}$ \\
\hline DISTRICT OF COLUMBIA \\
\hline Regulatory \\
\hline $\begin{array}{c}\text { Food Protection Branch } \\
\text { Dept. Of Consumer Regulatory Affairs } \\
\text { Business Inspection Div. } \\
\text { 614 H Street, NW Rm. } 616 \\
\text { Washington, DC } 20001 \\
\text { (202) 727-7250 } \\
\text { (FAX) 727-8030 }\end{array}$ \\
\hline Laboratory \\
\hline $\begin{array}{c}\text { Bur. Of Laboratories } \\
300 \text { Indiana Avenue, NW Washington, DC } 20001 \\
\text { (202) 727-0557 (FAX) 727-0563 }\end{array}$ \\
\hline FLORIDA \\
\hline Regulatory \\
\hline $\begin{array}{l}\text { Div. Of Dairy Industry } \\
\text { FL Dept. Of Agriculture \& Consumer Services } \\
3125 \text { Conner Blvd. }\end{array}$ \\
\hline
\end{tabular}




\begin{tabular}{|c|}
\hline $\begin{array}{l}\text { Tallahassee, FL 32399-1650 } \\
\text { (850) 487-1450 } \\
\text { (FAX) 922-9444 }\end{array}$ \\
\hline Laboratory \\
\hline $\begin{array}{l}\text { Central Dairy Laboratory } \\
\text { 3023 Lake Alfred Drive } \\
\text { Winter Haven, FL 33881-1438 } \\
\text { (863) 298-7748 } \\
\text { (FAX) 297-3091 } \\
\end{array}$ \\
\hline $\begin{array}{l}\text { Florida Dept. of Agriculture } \\
\text { 3125 Conner Blvd. } \\
\text { Tallahassee, FL 32399-1650 } \\
\text { (850) } 487-1490 \\
\text { (FAX) } 922-9444\end{array}$ \\
\hline GEORGIA \\
\hline Regulatory \\
\hline $\begin{array}{l}\text { Dairy Regulatory Div. } \\
\text { GA Dept. Of Agriculture } \\
19 \text { Martin Luther King Dr. } \\
\text { Capitol Square } \\
\text { Atlanta, GA 30334 } \\
\text { (404) 656-3625 } \\
\text { (FAX) 656-3723 }\end{array}$ \\
\hline Rating \\
\hline $\begin{array}{c}\text { IMS Rating \& Sampling Surveillance Programs Div. } \\
19 \text { Martin Luther King Dr. } \\
\text { Capitol Square } \\
\text { Atlanta, GA 30334 } \\
\text { (404) 656-3627 }\end{array}$ \\
\hline Laboratory \\
\hline $\begin{array}{c}\text { Laboratory Division } \\
\text { GA Dept. Of Agriculture } \\
19 \text { Martin Luther King Drive, Room } 610 \\
\text { Atlanta, GA 30334-2001 } \\
\text { (404) 656-3654 } \\
\text { (FAX) 656-9380 }\end{array}$ \\
\hline HAWAII \\
\hline Regulatory \\
\hline Sanitation Branch \\
\hline
\end{tabular}




\begin{tabular}{|c|}
\hline $\begin{array}{l}\text { Environmental Health Services Division } \\
\text { HI Dept. Of Health } \\
\text { 591 Ala Moana Blvd. } \\
\text { Honolulu, HI } 96813 \\
\text { (808) 586-8000 } \\
\text { (FAX) } 586-4729\end{array}$ \\
\hline Laboratory \\
\hline $\begin{array}{l}\text { HI State Dept. Of Health } \\
\text { State Laboratories Div. } \\
\text { Envm. Micro. Section } \\
2725 \text { Waimano Home Road } \\
\text { Pearl City, HI } 96782 \\
\text { (808) 453-6602 } \\
\text { (FAX) 453-6612 }\end{array}$ \\
\hline IDAHO \\
\hline Regulatory \\
\hline $\begin{array}{l}\text { Bureau of Dairying } \\
\text { ID Dept. Of Agriculture } \\
\text { 2270 Old Penitentiary Road } \\
\text { Boise, ID } 83712 \\
\text { (208) 332-8550 } \\
\text { (FAX) 334-4062 }\end{array}$ \\
\hline Laboratory \\
\hline $\begin{array}{l}\text { ID Dept. Of Agriculture } \\
\text { Animal Health Laboratory } \\
2230 \text { Old Penitentiary Road } \\
\text { Boise, ID } 83712 \\
\text { (208) 332-8570 } \\
\text { (FAX) 334-4619 }\end{array}$ \\
\hline ILLINOIS \\
\hline Regulatory \\
\hline $\begin{array}{l}\text { Div. Of Food, Drugs and Dairies } \\
\text { IL Dept. Of Public Health } \\
525 \text { W. Jefferson Street } \\
\text { Springfield, IL } 62761 \\
\text { (217) 785-2439 } \\
\text { (FAX) 782-0943 }\end{array}$ \\
\hline Laboratory \\
\hline Envm. Labs. Service \\
\hline
\end{tabular}




\begin{tabular}{|c|}
\hline $\begin{array}{l}\text { IL Dept. Of Public Health } \\
\text { Div. Of Laboratories } \\
825 \text { N. Rutledge Avenue } \\
\text { Springfield, IL 62794-9435 } \\
\text { (217) 782-6562 } \\
\text { (FAX) 524-7924 }\end{array}$ \\
\hline $\begin{array}{l}\text { Dept. Of Public Health } \\
\text { Chicago Laboratory } \\
2121 \text { W. Taylor Street } \\
\text { Chicago, IL } 60612 \\
\text { (312) 793-4760 } \\
\text { (FAX) 793-1322 }\end{array}$ \\
\hline INDIANA \\
\hline Regulatory \\
\hline $\begin{array}{c}\text { Dairy Division } \\
\text { IN State Board of Animal Health } \\
805 \text { Beachway Drive, Suite } 50 \\
\text { Indianapolis, IN 46224-7785 } \\
\text { (317) 227-0350 } \\
\text { Toll Free: } 877-747-3038 \times 350 \\
\text { (FAX) 227-0330 } \\
\end{array}$ \\
\hline Laboratory \\
\hline $\begin{array}{c}\text { Dairy Division } \\
\text { IN State Board of Animal Health } \\
805 \text { Beachway Drive, Suite } 50 \\
\text { Indianapolis, IN } 46224 \\
\text { (765) 935-6655 } \\
\text { (FAX) (317) 227-0330 }\end{array}$ \\
\hline Central Laboratory \\
\hline $\begin{array}{c}\text { Food Microbiology Dairy Products Testing Lab. } \\
\text { Consumer Health Labs. } \\
\text { IN State Dept. of Health } \\
\text { 1330 W. Michigan Street } \\
\text { P.O. Box 1964 } \\
\text { Indianapolis, IN 46206-1964 } \\
\text { (317) 233-8013 } \\
\text { (FAX) 233-8003 } \\
\end{array}$ \\
\hline IOWA \\
\hline Regulatory \\
\hline Dairy Products Control \\
\hline
\end{tabular}




\begin{tabular}{|c|}
\hline $\begin{array}{l}\text { IA Dept. Of Agriculture } \\
\text { Henry A. Wallace Bldg. } \\
\text { East 9th St. \& Grand Ave. } \\
\text { Des Moines, IA 50319 } \\
\text { (515) 281-3545 } \\
\text { (FAX) 281-8888 }\end{array}$ \\
\hline Rating \\
\hline $\begin{array}{l}\text { Div. Of Health Protection } \\
\text { IA Dept. Of Pub. Health } \\
\text { Lucas State Office Bldg. } \\
\text { Des Moines, IA 50319 } \\
\text { (515) 281-3773 } \\
\text { (FAX) 281-4529 }\end{array}$ \\
\hline Laboratory \\
\hline $\begin{array}{l}\text { Dairy Product Laboratory } \\
\text { IA Dept. Of Agriculture } \\
\text { Henry A. Wallace Bldg. } \\
\text { East 9th St. \& Grand Ave. } \\
\text { Des Moines, IA 50319 } \\
\text { (515) 242-6378 }\end{array}$ \\
\hline KANSAS \\
\hline Regulatory \\
\hline $\begin{array}{l}\text { Div. Of Inspections-Dairy } \\
\text { KS State Board of Agric. } \\
901 \text { S. Kansas Avenue 7th Floor E } \\
\text { Topeka, KS 66612-1272 } \\
\text { (785) 296-7020 } \\
\text { (FAX) 296-0673 }\end{array}$ \\
\hline Rating \\
\hline $\begin{array}{l}\text { Div. Of Inspection-Dairy } \\
\text { State Bd. Of Agriculture } \\
\text { 109 SW 9th Street } \\
\text { Topeka, KS 66612-1280 } \\
\text { (785) 296-3786 }\end{array}$ \\
\hline $\begin{array}{c}\text { Laboratory Division } \\
\text { KS Bd. Of Agriculture } \\
\text { 2524 SW 6th Avenue } \\
\text { Topeka, KS 66606-1703 } \\
\text { (785) 296-3301 } \\
\text { (FAX) 296-0253 }\end{array}$ \\
\hline
\end{tabular}




\begin{tabular}{|c|}
\hline KENTUCKY \\
\hline Regulatory \\
\hline $\begin{array}{c}\text { Milk Safety Branch } \\
\text { Cabinet for Human Resources/ } \\
\text { Dept. Of Health Services/ } \\
\text { Div. Of Envm. Health \& Community Safety } \\
\text { 275 E. Main Street } \\
\text { Frankfort, KY 40621-0001 } \\
\text { (502) 564-3340 } \\
\text { (FAX) } 564-8787\end{array}$ \\
\hline Laboratory \\
\hline $\begin{array}{l}\text { Div. of Laboratory Services } \\
100 \text { Sower Blvd. } \\
\text { Frankfort, KY } 40601 \\
\text { (502) } 564-4446 \text { x4426 } \\
\text { (FAX) } 564-7019\end{array}$ \\
\hline LOUISIANA \\
\hline Regulatory \\
\hline $\begin{array}{l}\text { Admin. Milk \& Dairy Product Unit } \\
\text { LA Dept. Of Health \& Hospitals } \\
6867 \text { Bluebonnet Road } \\
\text { Baton Rogue, LA 70810 } \\
\text { (225) 765-5022 } \\
\text { (FAX) 763-5552 }\end{array}$ \\
\hline Laboratory \\
\hline $\begin{array}{l}\text { LA Dept. Of Health \& Hospitals } \\
325 \text { Loyola Avenue, Rm } 709 \\
\text { New Orleans, LA } 70112 \\
\text { (504) 568-8229 } \\
\text { (FAX) 568-5393 }\end{array}$ \\
\hline MAINE \\
\hline Regulatory \\
\hline $\begin{array}{l}\text { Div. Of Animal Health \& Industry } \\
\text { ME Dept. Of Agriculture } \\
\text { State House Station } \# 28 \\
\text { Augusta, ME } 04333 \\
\text { (207) 287-3701 } \\
\text { (FAX) 287-7548 }\end{array}$ \\
\hline
\end{tabular}




\begin{tabular}{|c|}
\hline Laboratory \\
\hline $\begin{array}{c}\text { Div. Of Vet. Services } \\
\text { Dept. Of Agriculture } \\
\text { State House Station \#28 } \\
\text { Augusta, ME 04333-0028 } \\
\text { (207) 287-7623 } \\
\text { (FAX) 287-7548 }\end{array}$ \\
\hline MARYLAND \\
\hline Regulatory \\
\hline $\begin{array}{c}\text { Div. Of Milk Control } \\
\text { MD Dept. Of Health \& Mental Hygiene } \\
\text { 6 Saint Paul Street, Ste 1301 } \\
\text { Baltimore, MD 21202-1608 } \\
\text { (410) 767-8429 } \\
\text { (FAX) 333-8931 }\end{array}$ \\
\hline Laboratory \\
\hline $\begin{array}{c}\text { Lab. Administration } \\
\text { MD Dept. Of Health \& Mental Hygiene } \\
\text { 201 W. Preston Street } \\
\text { Baltimore, MD 21203-2355 } \\
\text { (410) 767-6146 } \\
\text { (FAX) 333-5403 }\end{array}$ \\
\hline MASSACHUSETTS \\
\hline Regulatory \\
\hline $\begin{array}{l}\text { (Raw Milk \& Farm Insp.) } \\
\text { Div. Of Dairy Services } \\
\text { MA Dept. Of Food \& Agric. } \\
\text { 251 Causeway St. Ste. 500 } \\
\text { Boston, MA 02114-2151 } \\
\text { (617) 626-1810 } \\
\text { (FAX) 626-1850 }\end{array}$ \\
\hline $\begin{array}{l}\text { (Pasteurized Milk \& Past. Plants) } \\
\text { Div. Of Food \& Drugs } \\
\text { MA State Dept. Of Health } \\
\text { 305 South Street } \\
\text { Jamaica Plain, MA 02130 } \\
\text { (617) } 983-6712 \\
\text { (FAX) } 983-6770\end{array}$ \\
\hline Laboratory \\
\hline
\end{tabular}




\begin{tabular}{|c|}
\hline $\begin{array}{l}\text { Div. Of Food \& Drugs } \\
\text { MA Dept. Of Public Health } \\
\text { 305 South Street } \\
\text { Jamaica Plain, MA 02130 } \\
\text { (617) 983-6616 } \\
\text { (FAX) 522-6618 } \\
\end{array}$ \\
\hline MICHIGAN \\
\hline Regulatory \\
\hline $\begin{array}{l}\text { Food and Dairy Division } \\
\text { MI Dept. Of Agriculture } \\
\text { P.O. Box } 30017 \\
\text { Lansing, MI } 48909 \\
\text { (517) 373-1060 } \\
\text { (FAX) 373-9742 }\end{array}$ \\
\hline Laboratory \\
\hline $\begin{array}{c}\text { MI State LEO } \\
\text { MI Dept. Of Agriculture } \\
\text { Laboratory Division } \\
\text { 8585 Anderson Highway } \\
\text { Vermontville, MI } 49096 \\
\text { (517) 726-1541 } \\
\text { (FAX) 726-1541 } \\
\end{array}$ \\
\hline MINNESOTA \\
\hline Regulatory \\
\hline $\begin{array}{l}\text { MN Dept. Of Agriculture } \\
\text { Dairy \& Food Inspection Div. } \\
90 \text { W. Plato Blvd. } \\
\text { Saint Paul, MN 55107 } \\
\text { (651) 296-1586 } \\
\text { (FAX) 297-5176 }\end{array}$ \\
\hline Laboratory \\
\hline $\begin{array}{c}\text { MN Dept. Of Agriculture } \\
90 \text { W Plato Blvd. } \\
\text { Saint Paul, MN 55107-2004 } \\
\text { (651) 297-1901 } \\
\text { (FAX) 297-8787 }\end{array}$ \\
\hline MISSISSIPPI \\
\hline Regulatory \\
\hline
\end{tabular}




\begin{tabular}{|c|}
\hline $\begin{array}{l}\text { Dairy Farm Inspection Div. A101 } \\
\text { MS State Dept. Of Health } \\
570 \text { E. Woodrow Wilson } \\
\text { PO Box 1700 } \\
\text { Jackson, MS 39215-1700 } \\
\text { (601) 576-7628 } \\
\text { (FAX) 576-7632 }\end{array}$ \\
\hline $\begin{array}{c}\text { Bottled Water \& Milk Plant Inspection Br. } \\
\text { MS Dept. Of Health } \\
570 \text { E. Woodrow Wilson } \\
\text { PO Box } 1700 \\
\text { Jackson, MS } 39215-1700 \\
\text { (601) 576-7628 } \\
\text { (FAX) 576-7632 }\end{array}$ \\
\hline Laboratory \\
\hline $\begin{array}{l}\text { Bur. Of Public Health Labs } \\
\text { MS Board of Health } \\
2423 \text { N. State Street } \\
\text { PO Box 1700 } \\
\text { Jackson, MS 39215-1700 } \\
\text { (601) 576-7582 } \\
\text { (FAX) 576-7720 }\end{array}$ \\
\hline MISSOURI \\
\hline Regulatory \\
\hline $\begin{array}{l}\text { MO State Milk Board } \\
\text { 915-C Leslie Blvd. } \\
\text { Jefferson City, MO 65101 } \\
\text { (573) 751-3830 } \\
\text { (FAX) 751-2527 } \\
\end{array}$ \\
\hline Rating \\
\hline $\begin{array}{l}\text { MO Dept. Of Health } \\
1730 \text { East Elm Street } \\
\text { Jefferson City, MO 65102 } \\
\text { (573) } 751-6095 \\
\text { (FAX) 526-7377 }\end{array}$ \\
\hline Laboratory \\
\hline $\begin{array}{l}\text { State Public Health Lab. Dept. of Health } \\
307 \text { W. McCarthy Street } \\
\text { Jefferson City, MO 65102-0570 } \\
\text { (573) 751-7242 } \\
\text { (FAX) 751-7219 }\end{array}$ \\
\hline
\end{tabular}




\begin{tabular}{|c|}
\hline MONTANA \\
\hline Regulatory \\
\hline $\begin{array}{l}\text { Dairy \& Eggs. Bureau } \\
\text { MT Dept. Of Livestock } \\
\text { Livestock Bldg. } \\
\text { Capitol Station } \\
\text { Helena, MT 59601 } \\
\text { (406) 444-9761 } \\
\text { (FAX) 444-1929 }\end{array}$ \\
\hline Laboratory \\
\hline $\begin{array}{l}\text { Diagnostic Laboratories } \\
\text { MT Dept. Of Livestock } \\
\text { P.O. Box 997 } \\
\text { Bozeman, MT 59771-0997 } \\
\text { (406) 994-6352 } \\
\text { (FAX) 994-6344 }\end{array}$ \\
\hline NEBRASKA \\
\hline Regulatory \\
\hline $\begin{array}{l}\text { Bur. Of Dairies \& Foods } \\
\text { NE Dept. Of Agriculture } \\
\text { 301 Centennial Mall South } \\
\text { Lincoln, NE 68509-5064 } \\
\text { (402) 471-2536 } \\
\text { (FAX) 471-2759 }\end{array}$ \\
\hline Laboratory \\
\hline $\begin{array}{c}\text { Laboratory Division } \\
\text { NE Dept. Of Agriculture } \\
3703 \text { South 14th Street } \\
\text { Lincoln, NE 68502-5399 } \\
\text { (402) 471-2176 } \\
\text { (FAX) 471-0091 }\end{array}$ \\
\hline NEVADA \\
\hline Regulatory \\
\hline $\begin{array}{l}\text { NV State Dairy Commission } \\
4600 \text { Kietzke Lane, Suite A-107 } \\
\text { Reno, NV 89502 } \\
\text { (775) 688-1211 x25 } \\
\text { (FAX) 688-1218 }\end{array}$ \\
\hline
\end{tabular}




\begin{tabular}{|c|}
\hline Laboratory \\
\hline $\begin{array}{l}\text { Bur. Of Laboratories } \\
\text { NV Dept. Of Human Resources } \\
1660 \text { N. Virginia Street } \\
\text { Reno, NV } 89503 \\
\text { (775) 688-1335 x240 } \\
\text { (FAX) 688-1460 }\end{array}$ \\
\hline NEW HAMPSHIRE \\
\hline Regulatory \\
\hline $\begin{array}{c}\text { Dairy Sanitation Program } \\
\text { Bur. Of Food Protection } \\
\text { NH Dept. Of Health \& Human Services } \\
\text { Brown Building } \\
129 \text { Pleasant Street } \\
\text { Concord, NH } 03302 \\
\text { (603) 271-4673 } \\
\text { (FAX) 271-4859 }\end{array}$ \\
\hline Laboratory \\
\hline $\begin{array}{l}\text { State Public Health Lab. } \\
6 \text { Hazen Drive } \\
\text { Concord, NH 03301-6527 } \\
\text { (603) 271-4665 } \\
\text { (FAX) 271-4783 }\end{array}$ \\
\hline NEW JERSEY \\
\hline Regulatory \\
\hline $\begin{array}{l}\text { Food \& Milk Program } \\
\text { NJ Dept. Of Health } \\
\text { 3635 Quaker Bridge Road } \\
\text { PO Box 369 } \\
\text { Trenton, NJ 08625-0369 } \\
\text { (609) 588-3123 } \\
\text { (FAX) 588-7431 } \\
\end{array}$ \\
\hline Laboratory \\
\hline $\begin{array}{l}\text { NJ Dept. Of Health } \\
\text { Div. Of Laboratories } \\
\text { John Fitzh Plaza, CN-361 } \\
\text { Trenton, NJ } 08625 \\
\text { (609) 292-9455 } \\
\text { (FAX) 633-9601 }\end{array}$ \\
\hline
\end{tabular}




\begin{tabular}{|c|}
\hline \multirow{2}{*}{$\begin{array}{c}\text { NEW MEXICO } \\
\text { Regulatory }\end{array}$} \\
\hline \\
\hline $\begin{array}{c}\text { Dairy Division } \\
\text { NM Dept. Of Agriculture } \\
\text { 2604 Aztec N.E. } \\
\text { Albuquerque, NM } 87107 \\
\text { (505) 841-9425 } \\
\text { (FAX) } 841-9426\end{array}$ \\
\hline Laboratory \\
\hline $\begin{array}{c}\text { Science Lab. Division } \\
\text { P.O. Box } 4700 \\
\text { Albuquerque, NM } 87196-4700 \\
\text { (505) } 841-2537 \\
\text { (FAX) } 841-2543\end{array}$ \\
\hline NEW YORK \\
\hline Regulatory \\
\hline $\begin{array}{c}\text { Div. Of Milk Control } \\
\text { NY Dept. Of Agriculture \& Markets } \\
\text { Capital Plaza, 1 Winners Circle } \\
\text { Albany, NY } 12235 \\
\text { (518) 457-1772 } \\
\text { (FAX) 485-8730 }\end{array}$ \\
\hline Laboratory \\
\hline $\begin{array}{c}\text { Div. Of Milk Control } \\
\text { NY Dept. Of Agriculture \& Markets } \\
\text { Capital Plaza, 1 Winners Circle } \\
\text { Albany, NY 12235-0001 } \\
\text { (518) 457-8872 } \\
\text { (FAX) 485-8730 }\end{array}$ \\
\hline "NORTH CAROLINA \\
\hline Regulatory \\
\hline $\begin{array}{c}\text { NC Envm. Health Services Section } \\
8297 \text { Hagers Ferry Road } \\
\text { Denver, NC 28037 } \\
\text { (704) 483-6218 } \\
\text { (FAX) 483-6218 }\end{array}$ \\
\hline Laboratory \\
\hline
\end{tabular}




\begin{tabular}{|c|}
\hline $\begin{array}{c}\text { Envm. Sciences Section/ } \\
\text { Lab. Services Div. } \\
\text { NC Dept. Of Envm. Health \& Natural Resources } \\
\text { P.O. Box 28047 } \\
\text { Raleigh, NC 27611-8047 } \\
\text { (919) 733-7308 } \\
\text { (FAX) 733-8695 } \\
\end{array}$ \\
\hline NORTH DAKOTA \\
\hline Regulatory \\
\hline $\begin{array}{l}\text { ND Dept. Of Agriculture } \\
\text { State Capitol } \\
\text { Bismarck, ND 58505 } \\
\text { (701) } 328-4761 \\
\text { (FAX) } 328-4567\end{array}$ \\
\hline Laboratory \\
\hline $\begin{array}{l}\text { ND Dept. Of Health } \\
\text { Div. Of Microbiology } \\
\text { 1205 Avenue A West } \\
\text { Bismarck, ND 58502-5520 } \\
\text { (701) } 328-5262 \\
\text { (FAX) 328-5270 }\end{array}$ \\
\hline OHIO \\
\hline Regulatory \\
\hline $\begin{array}{c}\text { Dairy Division } \\
\text { OH Dept. Of Agriculture } \\
\text { 8995 E. Main Street } \\
\text { Reynoldsburg, OH 43068-3399 } \\
\text { (614) 466-5550 } \\
\text { (FAX) 728-2652 } \\
\end{array}$ \\
\hline $\begin{array}{c}\text { Consumer Analytical Lab. } \\
\text { OH Dept. Of Agriculture } \\
\text { 8995 E. Main Street } \\
\text { Reynoldsburg, OH 43068-3399 } \\
\text { (614) 728-6318 } \\
\text { (FAX) 728-6322 }\end{array}$ \\
\hline OKLAHOMA \\
\hline Regulatory \\
\hline
\end{tabular}




\begin{tabular}{|c|}
\hline $\begin{array}{c}\text { OK Dept. Of Agriculture } \\
\text { Dairy Service Division } \\
\text { P.O. Box 528804 } \\
\text { Oklahoma City, OK 73152-8804 } \\
\text { (405) 522-6130 } \\
\text { (FAX) 522-0756 }\end{array}$ \\
\hline Laboratory \\
\hline $\begin{array}{l}\text { Laboratory Services } \\
\text { OK Dept. Of Agriculture } \\
\text { 440 S. Houston Ave. Room } 10 \\
\text { Tulsa, OK } 74127 \\
\text { (918) 581-2014 } \\
\text { (FAX) 581-2820 }\end{array}$ \\
\hline OREGON \\
\hline Regulatory \\
\hline $\begin{array}{c}\text { Food \& Dairy Division } \\
\text { OR Dept. Of Agriculture } \\
\text { Agriculture Bldg. 635 Capitol Street, NE } \\
\text { Salem, OR } 97301 \\
\text { (503) 986-4720 } \\
\text { (FAX) 986-4729 }\end{array}$ \\
\hline Laboratory \\
\hline $\begin{array}{c}\text { Laboratory Services } \\
\text { Food \& Dairy Division } \\
\text { OR Dept. Of Agriculture } \\
1207 \text { NW Naito Parkway, Suite } 204 \\
\text { Portland, OR 97209 } \\
\text { (503) 872-6644 } \\
\text { (FAX) 872-6615 }\end{array}$ \\
\hline PENNSYLVANIA \\
\hline Regulatory \\
\hline $\begin{array}{l}\text { Div. Of Milk Sanitation } \\
\text { Bureau of Food Safety } \\
\text { PA Dept. Of Agriculture } \\
\text { 2301 N. Cameron Street } \\
\text { Harrisburg, PA } 17110 \\
\text { (717) } 787-4316 \\
\text { (FAX) } 787-1873\end{array}$ \\
\hline Laboratory \\
\hline
\end{tabular}




\begin{tabular}{|c|}
\hline $\begin{array}{l}\text { PA Dept. Of Agriculture } \\
\text { Bur. Of Safety Chemical Services } \\
\text { 2301 N. Cameron Street } \\
\text { Harrisburg, PA 17110-9408 } \\
\text { (FAX) (717) 787-1873 }\end{array}$ \\
\hline $\begin{array}{l}\text { PA Dept. Of Agriculture } \\
\text { Bur. Of Food Safety, Reg. IV } \\
5349 \text { William Flynn Hwy. } \\
\text { Gibsonia, PA 15044 } \\
\text { (724) 443-1585 } \\
\text { (FAX) 443-8150 }\end{array}$ \\
\hline PUERTO RICO \\
\hline Regulatory \\
\hline $\begin{array}{c}\text { Programme Higiene de Leche } \\
\text { Call Box 70184 } \\
\text { San Juan, PR 00936 } \\
\text { (787) 274-7806/7804 } \\
\text { (FAX) 758-6285 }\end{array}$ \\
\hline Laboratory \\
\hline $\begin{array}{c}\text { Departmento de Salud } \\
\text { Instituto de Labratorios } \\
\text { Edificio A/Call Box } 70184 \\
\text { San Juan, PR 00936 } \\
\text { (787) 274-7705 } \\
\text { (FAX) 759-6210 }\end{array}$ \\
\hline RHODE ISLAND \\
\hline Regulatory \\
\hline $\begin{array}{l}\text { Envm. Health Food Specialist } \\
\text { RI Dept. Of Health } \\
\text { Office of Food Protection } \\
\text { Dairy Industry Program } \\
3 \text { Capitol Hill } \\
\text { Providence, RI 02908-5097 } \\
\text { (401) 222-3430 x2421 } \\
\text { (FAX) 222-4775 }\end{array}$ \\
\hline Laboratory \\
\hline $\begin{array}{l}\text { Sanitary Micro. Laboratory } \\
\text { Laboratory Services } \\
\text { Laboratory Health Bldg. }\end{array}$ \\
\hline
\end{tabular}




\begin{tabular}{|c|}
\hline $\begin{array}{c}50 \text { Orms Street } \\
\text { Providence, RI 02904-2284 } \\
\text { (401) 222-5588 } \\
\text { (FAX) 222-6985 }\end{array}$ \\
\hline SOUTH CAROLINA \\
\hline Regulatory \\
\hline $\begin{array}{l}\text { Dairy Foods \& Soft Drinks Program Protection } \\
\text { Bur. Of Envm. Health } \\
\text { SC Dept. Of Health \& Environmental Control } \\
2600 \text { Bull Street } \\
\text { Columbia, SC 29201 } \\
\text { (803) 896-0644 } \\
\text { (FAX) 896-0645 }\end{array}$ \\
\hline Laboratory \\
\hline $\begin{array}{l}\text { Div. Of Envm. Health Labs. } \\
\text { SC Dept. Of Health \& Environmental Control } \\
\text { 8231 Parklane Road } \\
\text { Columbia, SC 29223 } \\
\text { (803) 896-0871 } \\
\text { (FAX) 896-0983 } \\
\end{array}$ \\
\hline SOUTH DAKOTA \\
\hline Regulatory \\
\hline $\begin{array}{l}\text { Office of Dairy Inspection } \\
\text { Div. Of Regulatory Services } \\
\text { 523 E. Capitol Foss Bldg. 3rd Floor } \\
\text { Pierre, SD 57501-3182 } \\
\text { (605) 773-3724 } \\
\text { (FAX) 773-3481 }\end{array}$ \\
\hline Laboratory \\
\hline $\begin{array}{c}\text { State Dairy Laboratory } \\
\text { Dairy Science Dept. } \\
\text { Dairy Microbiology Bldg. } \\
\text { SD State University, Box } 2104 \\
\text { Brookings, SD 57007-0647 } \\
(605) 688-5491 \\
\text { (FAX) 688-6276** call before faxing }\end{array}$ \\
\hline TENNESSEE \\
\hline Regulatory \\
\hline
\end{tabular}




\begin{tabular}{|c|}
\hline $\begin{array}{c}\text { Div. Of Regulatory Services } \\
\text { TN Dept. Of Agriculture } \\
\text { Ellington Agric. Center, Box } 40627 \\
\text { Melrose Station } \\
\text { Nashville, TN } 37204 \\
\text { (615) 837-5150 } \\
\text { (FAX) } 837-5335\end{array}$ \\
\hline Laboratory \\
\hline $\begin{array}{l}\text { TN Dept. Of Agriculture } \\
\text { Div. Of Technical/Services } \\
\text { L.H. Ivy Building } \\
\text { P.O. Box } 40627 \\
\text { Melrose Station } \\
\text { Nashville, TN } 37204 \\
\text { (615) } 837-5265 \\
\text { (FAX) } 837-5516\end{array}$ \\
\hline TEXAS \\
\hline Regulatory \\
\hline $\begin{array}{l}\text { Div. Of Milk \& Dairy Products } \\
\text { TX Dept. Of Health } \\
\text { 1100 W. 49th Street } \\
\text { Austin, TX 78756 } \\
\text { (512) 719-0260 } \\
\text { (FAX) 719-0250 }\end{array}$ \\
\hline Laboratory \\
\hline $\begin{array}{l}\text { Bur. Of Laboratories } \\
\text { TX Dept. Of Health } \\
\text { 1100 W. 49th Street } \\
\text { Austin, TX 78756-3194 } \\
\text { (512) 458-7318 x7585 } \\
\text { (FAX) 458-7452 } \\
\end{array}$ \\
\hline UTAH \\
\hline Regulatory \\
\hline $\begin{array}{l}\text { Div. Of Food \& Consumer Service } \\
\text { UT Dept. Of Agriculture } \\
350 \text { N. Redwood Road, Box } 146500 \\
\text { Salt Lake City, UT } 84114-6500 \\
\text { (801) 538-7150 } \\
\text { (FAX) 538-7126 }\end{array}$ \\
\hline Laboratory \\
\hline
\end{tabular}




\begin{tabular}{|c|}
\hline $\begin{array}{l}\text { Div. Of Agriculture Labs } \\
\text { UT Dept. Of Agriculture } \\
350 \text { N. Redwood Road, Suite } 312 \\
\text { Salt Lake City, UT } 84116 \\
\text { (801) 538-7129 } \\
\text { (FAX) 538-7126 } \\
\end{array}$ \\
\hline VERMONT \\
\hline Regulatory \\
\hline $\begin{array}{c}\text { Dairy Section } \\
\text { VT Dept. Of Agriculture } \\
116 \text { State Street, Drawer } 20 \\
\text { Montpelier, VT 05602-2901 } \\
\text { (802) 828-2433 } \\
\text { (FAX) } 828-5983\end{array}$ \\
\hline Laboratory \\
\hline $\begin{array}{l}\text { VT Dept. Of Agriculture } \\
103 \text { S. Main Street } \\
\text { Waterbury, VT } 05676 \\
\text { (802) 244-4510 } \\
\text { (FAX) 241-3008 }\end{array}$ \\
\hline VIRGINIA \\
\hline Regulatory \\
\hline $\begin{array}{c}\text { (Raw Milk Farm Insp.) } \\
\text { Dept. Of Agriculture \& Consumer Services } \\
\text { P.O. Box } 1163 \\
\text { Richmond, VA } 23218 \\
\text { (804) 786-8899 } \\
\text { (FAX) 371-7792 }\end{array}$ \\
\hline $\begin{array}{c}\text { (Past. Plants \& Past. Milk \& Rating) } \\
\text { Division of Food and Environmental Services } \\
\text { VA State Health Dept. } \\
1500 \text { E. Main Street Station, Suite } 115 \\
\text { Richmond, VA 23218-2448 } \\
\text { (804) 225-4026 } \\
\text { (FAX) 225-4003 } \\
\end{array}$ \\
\hline (Raw Milk) \\
\hline $\begin{array}{c}\text { VA Dept. Of Agriculture } \\
\text { Warrenton Animal Health, Lab. \& Consumer Services }\end{array}$ \\
\hline
\end{tabular}




\begin{tabular}{|c|}
\hline $\begin{array}{l}272 \text { Academy Hill Road } \\
\text { Warrenton, VA } 20186 \\
\text { (540) 347-6385 } \\
\text { (FAX) 347-6404 }\end{array}$ \\
\hline $\begin{array}{l}\text { VA Dept. Of Agriculture } \\
\text { \& Consumer Services } \\
\text { 250 Cassell Road } \\
\text { Wytheville, VA 24382 } \\
\text { (276) 228-5501 } \\
\text { (FAX) 223-1961 }\end{array}$ \\
\hline $\begin{array}{c}\text { (Pasteurization) } \\
\text { VA Dept. of General Services } \\
\text { Div. of Consolidated Lab. Service } \\
\text { 1 North 14th Street, Room } 442 \\
\text { Richmond, VA 23219-3691 } \\
\text { (804) 371-2871 } \\
\text { (FAX) 371-0666 }\end{array}$ \\
\hline WASHINGTON \\
\hline Regulatory \\
\hline $\begin{array}{l}\text { WA Dept. Of Agriculture } \\
\text { Food Safety Program } \\
\text { 1111 Washington Street } \\
\text { P.O. Box 42560 } \\
\text { Olympia, WA 98504-2560 } \\
\text { (360) 902-1905 } \\
\text { (FAX) 902-2087 }\end{array}$ \\
\hline Laboratory \\
\hline $\begin{array}{c}\text { Laboratory Service } \\
\text { WA State Dept. Of Agric. } \\
\text { 3939 Cleveland Avenue, SE } \\
\text { Olympia, WA } 98501 \\
\text { (360) 753-3106 } \\
\text { (FAX) 753-5047 }\end{array}$ \\
\hline WEST VIRGINIA \\
\hline Regulatory \\
\hline $\begin{array}{l}\text { WV Bureau of Public Health } \\
\text { Office of Envm. Health } \\
\text { Public Health San. Div. } \\
\text { 815 Quarrier Street, Suite } 418 \\
\text { Charleston, WV 25301 }\end{array}$ \\
\hline
\end{tabular}




\begin{tabular}{|c|}
\hline $\begin{array}{l}(304) 558-2981 \\
\text { (FAX) 558-0691 }\end{array}$ \\
\hline Laboratory \\
\hline $\begin{array}{l}\text { Offices of Lab. Services } \\
16711 \text { th Avenue } \\
\text { S. Charleston, WV } 25303 \\
\text { (304) 558-3530 } \\
\text { (FAX) 558-2006 }\end{array}$ \\
\hline $\begin{array}{l}\text { Regulatory Protection Div. } \\
\text { WV Dept. Of Agriculture } \\
1900 \text { Kanawha Blvd. E } \\
\text { Charleston, WV 25305-0177 } \\
\text { (304) 558-2208 } \\
\text { (FAX) 558-3594 } \\
\end{array}$ \\
\hline WISCONSIN \\
\hline Regulatory \\
\hline $\begin{array}{c}\text { Food Division, WI Dept. Of Agriculture } \\
\text { P.O. Box } 8911 \\
\text { 2811 Agricultural Drive } \\
\text { Madison, WI 53708-8911 } \\
\text { (608) 224-4701 } \\
\text { (FAX) 224-4710 }\end{array}$ \\
\hline Laboratory \\
\hline $\begin{array}{l}\text { Food Safety \& Inspection Bureau, WI Dept. Of Agriculture } \\
\text { Trade \& Consumer Protection } \\
\text { P.O. Box 8911 } \\
\text { 2811 Agricultural Drive } \\
\text { Madison, WI 53708-8911 } \\
\text { (608) 224-4712 } \\
\text { (FAX) 224-4710 }\end{array}$ \\
\hline WYOMING \\
\hline Regulatory \\
\hline $\begin{array}{l}\text { Food \& Drug Standards } \\
\text { WY Dept. Of Agriculture } \\
2219 \text { Carey Avenue } \\
\text { Cheyenne, WY } 82002 \\
\text { (307) 777-7321 } \\
\text { (FAX) 777-6593 } \\
\end{array}$ \\
\hline Laboratory \\
\hline
\end{tabular}


Analytical Service, WY Dept. Of Agriculture

1174 Snowy Range Road

Laramie, WY 82070

(307) 742-2984

(FAX) 742-2156 


\title{
THE ENVIRONMENTAL PROTECTION AGENCY'S (EPA)
}

List of State Certification Officers for Drinking Water Laboratories, Updated May 2001 (http://www.epa.gov/safewater/faq/sco.html)

\author{
ALABAMA \\ (334) $271-7773$ \\ Department of Environmental Management \\ Drinking Water Branch \\ PO Box 301463 \\ Montgomery, AL 36130-1463

\section{ALASKA} \\ (907) 790-2169 \\ Department of Environmental Conservation \\ State Chemistry Laboratory \\ 10107 Bentwood Place \\ Juneau, AK 99801

\section{ARIZONA} \\ (602) 364-0746 \\ Department of Health Services \\ Laboratory Licensure and Certification \\ 1740 W. Adams \\ Suite $203 \mathrm{~N}$ \\ Phoenix, AZ 85007
}

\section{ARKANSAS}

refer to the "Laboratories-Analytical" section of the yellow pages

\section{CALIFORNIA}

(510) 540-2800

Laboratory Certification Office

2151 Berkeley Way, Annex 2

Berkeley, CA 94704

COLORADO

(303) 692-3291

Department of Health and Environment

Lab Certification Unit

8100 East Lowry Blvd.

Denver, CO 80230

\section{CONNECTICUT}

(860) 509-7389

Department of Public Health

Environmental Health - Laboratory Certification Program

P.O. Box 340308, Mail Stop \# 51 LAB

410 Capital Avenue

Hartford, CT 06106 


\section{DELAWARE}

Chemicals, Microbes \& Radionuclides: (302) 739-5410

Radon: (302) 739-4731

Health Services Protection

Public Water System Supervision Program

Federal Street

P.O. Box 637

Dover, DE 19903

\section{FLORIDA}

Chemicals \& Microbes: (904) 791-1599

Radionuclides: (407) 297-2095

Radon: (850) 245-4288 or (800) 543-8279

Department of Health and Rehabilitative Services

1217 N. Pearl Street

Jacksonville, FL 32202

\section{GEORGIA}

(404) 656-4807

Georgia Department of Natural Resources

Drinking Water Permitting Program

Floyd Towers, East Suite 1362

205 Butler Street, SE

Atlanta, GA 30334

\section{GUAM}

(671) 475-1664

Monitoring Services Division

Guam Environmental Protection Agency

15-6101 Mariner Avenue, Tiyan,

Barrigada, Guam 96913

(mailing address:

PO Box 22439 GMF,

Barrigada, Guam 96921)

\section{HAWAII}

(808) 586-4258

Department of Health

919 Ala Moana Blvd, Room 308

Honolulu, HI 96814

\section{IDAHO}

(208) 334-2235 ext. 270

Manager of Laboratory Improvement

State Health Department

2220 Old Penitentiary Road

Boise, ID 83712-8299

\section{ILLINOIS}

Chemicals: (217) 782-6455

Illinois Environmental Protection Agency

P.O. Box 19276 
1021 North Grand Ave., East

Springfield, IL 62794-9276

Microbes: (217) 782-6562

Department of Public Health

P.O. Box 19435

825 North Rutlidge

Springfield, IL 62794-9435

Radon: (312) 793-4760 or (800) 325-1245

Dept. of Public Health

Division of Laboratories

2121 West Taylor St.

Chicago, IL 60612-4285

\section{INDIANA}

Chemicals \& Radionuclides: (317) 233-8071

Microbes: (317) 233-8072

Department of Health

635 North Barnhille Drive

Indianapolis, IN 46202

Radon: (317) 233-7146

Department of Health

2 North Meridian

Indianapolis, IN 46204

\section{IOWA}

(515) 725-0341

Department of Natural Resources

Water Quality Bureau

401 SW 7th St., Suite M

Des Moines, IA 50309-4611

\section{KANSAS}

(785) 296-1639

Health and Environmental Laboratory

Department of Health and Environment

Forbes Field, Building \#740

Topeka, KS 66620-0001

\section{KENTUCKY}

(502) 564-6121

Department of Environmental Protection

100 Sower Blvd., Suite 104

Frankfort, KY 40601

\section{LOUISIANA}

(504)568-3455

Division of Laboratory Services

Department of Health and Human Services 
325 Loyola Avenue, Room 709

New Orleans, LA 70112

\section{MAINE}

(207) 287-1929

Health and Environment Testing Laboratory

State House, Station 12

221 State Street

Augusta, ME 04333

\section{MARYLAND}

(410) 631-3706

Department of the Environment

Public Drinking Water Program

2500 Broening Highway

Baltimore, MD 21224

\section{MASSACHUSETTS}

Chemicals \& Radionuclides: (978) 682-5237 ext: 331

Microbes: (978) 682-5237 ext. 333

Radon: (617) 983-6870

Department of Environmental Protection

William X. Wall Experiment Station

37 Shattuck Street

Lawrence, MA 01843

\section{MICHIGAN}

(517) 335-8812

Department of Environmental Quality

Drinking Water and Radiological Protection Division

3350 Martin Luther King, Jr. Boulevard

Building \#44, 3rd floor

Lansing, Ml 48909-2933

\section{MINNESOTA}

Chemicals \& Microbes: (612) 676-5200

Radon: (800) 383-9808

Department of Health

717 Delaware Street, S. E.

P. O. Box 9441

Minneapolis, MN 55440-9441

\section{MISSISSIPPI}

Chemicals \& Microbes: (601) 576-7518

Radon \& Radionuclides: (601) 987-6893 or (800) 626-7739

State Department of Health

P.O. Box 1700

Jackson, MS 39215-1700

\section{MISSOURI}

(573) 751-1077 or (800) 334-6946

Public Drinking Water Program 
Department of Natural Resources

P.O. Box 176

Jefferson City, MO 65102

\section{MONTANA}

(406) 444-4400

Department of Environmental Quality

Community Services Bureau, Public Water Supply Program

1520 East 6th Avenue, PO Box 200901

Helena, MT 59620-0901

\section{NEBRASKA}

(402) 471-2122

Division of Laboratories

Department of Health and Human Services

P.O. Box 2755

Lincoln, NE 68502

\section{NEVADA}

Chemicals \& Microbes: (775) 688-2888

Radon: (775) 687-5394

Bureau of Licensure and Certification

State Health Department

1755 East Plumb Lane, Suite 241

Reno, NV 89502

\section{NEW HAMPSHIRE}

(603) 271- 3503

Department of Environmental Services Laboratory

6 Hazen Drive

P.O. Box 95

Concord, $\mathrm{NH} 03301$

\section{NEW JERSEY}

(609) 292-3950

Office of Quality Assurance

Department of Environmental Protection

P.O. Box 424

Trenton, NJ 08625

\section{NEW MEXICO}

(505) 827-7536

Drinking Water Bureau

Environment Department

525 Camino de las Marquez, Suite 4

Santa Fe, NM 87501

\section{NEW YORK}

(518) 485-5570

Department of Health

Wadsworth Center

Empire State Plaza 
P.O. Box 509

Albany, NY 12201-0509

NORTH CAROLINA

Chemicals \& Microbes: (919) 733-7308

Radionuclides \& Radon: (919) 571-4141

Department of Health \& Human Services

Lab Services

P.O. Box 28047

Raleigh, NC 27611

\section{NORTH DAKOTA}

(701) 328-6172

Department of Health

Division of Chemicals - P.O. Box 937

Division of Microbes - P.O. Box 5520

Bismarck, ND 58502

\section{$\mathrm{OHIO}$}

(614) 644-2752

Ohio Environmental Protection Agency

P.O. Box 1049

$122 \mathrm{~S}$. Front Street

Columbus, OH 43215-1049

\section{OKLAHOMA}

Chemicals \& Microbes: (405) 702-1024

Radon: (405) 702-1000

Dept. of Environmental Quality

Box 1677

Oklahoma City, OK 73101-1677

\section{OREGON}

(503) 229-5505

State Public Health Laboratory

1717 South West 10th Avenue

Portland, OR 97201

\section{PACIFIC ISLANDS}

(415)744-1594

U.S. EPA Region 9

Pacific Insular Area Programs

75 Hawthorne Street

San Francisco, CA 94105

PENNSYLVANIA

(717) 783-7150

Department of Environmental Protection

P.O. Box 1467

Harrisburg, PA 17105-1467 


\section{PUERTO RICO}

Microbes: (787) 274-7711

Chemicals \& Radionuclides: (787) 274-7713

Department of Health

Edificio a Centro Medico

P.O. Box 70184

San Juan, PR 00936

\section{RHODE ISLAND}

(401) 222-5600 - ask for Ewa King

Department of Health

50 Orms Street

Providence, RI 02904

\section{SOUTH CAROLINA}

(803) 896-0970

Department of Health \& Environmental Control

P.O. Box 72

State Park, SC 29147

\section{SOUTH DAKOTA}

(605) 773-3368 or (800) 738-2301

Department of Health

615 East 4th Street

Pierre, SD 57501-1700

\section{TENNESSEE}

(615) 532-0183

Department of Environmental Conservation

6th Floor, L\&C Tower

401 Church Street

Nashville, TN 37243-1549

\section{TEXAS}

(512) 239-3518

TNRCC, MC 176

P. O. Box 13087

Austin, TX 78711-3087

\section{UTAH}

(801) 584-8459

State Health Laboratory

46 North Medical Drive

Salt Lake City, UT 84113-1105

\section{VERMONT}

(802) 863-7335

Department of Health

P.O. 1125

Burlington, VT 05402-1125 


\section{VIRGIN ISLANDS}

(340) 774-3320 ×3

Department of Planning and Natural Resources

Wheatley Center 2

St. Thomas, Virgin Islands 00802

\section{VIRGINIA}

(804) 786-7905

Laboratory Certification Officer

One North 14th Street

Richmond, VA 23219

\section{WASHINGTON}

(206) 361-2820

Department of Health

Health Laboratory

Drinking Water Laboratory Certification Office

1610 North East 150th Street

Shoreline, WA 98155

\section{WEST VIRGINIA}

Chemicals \& Radionuclides: (304) 558-2782

Office of Laboratory Services

Bureau of Public Health

4710 Chimney Dr.

Charleston, WV 25302

Microbes: (304) 558-3530

Office of Laboratory Services

State Health Department

16711 th Avenue

South Charleston, WV 25303

\section{WISCONSIN}

(608) 267-7633

Laboratory Certification Program

Department of Natural Resources

P.O. Box 7291

Madison, WI 53707-7921

\section{WYOMING}

(303) 312-6985

Drinking Water Laboratory Certification program

U.S. EPA Region 8

999 18th Street, Suite 300

Denver Federal Center

Denver, CO 80202 


\title{
LIST OF STATE CONTACT INFORMATION AVAILABLE FROM FEDERAL AGENCIES
}

\author{
THE CONSUMER PRODUCT SAFETY COMMISSION \\ List of Product Safety State Contacts \\ Is Available at: \\ http://www.cpsc.gov/businfo/state/state.html
}

THE ENVIRONMENTAL PROTECTION AGENCY'S (EPA)

State and Territorial Underground Storage Tank (UST) Program Directory

Is Available at:

http://www.epa.gov/swerust1/states/statcon1.htm

THE ENVIRONMENTAL PROTECTION AGENCY'S (EPA)

List of State Pesticide Regulatory Agencies

Is Available at:

http://npic.orst.edu/state1.htm

THE FOOD AND DRUG ADMINISTRATION'S (FDA)

DIRECTORY OF STATE AND LOCAL OFFICIALS

2002 EDITION

Is Available at:

http://www.fda.gov/ora/fed_state/directorytable.htm

THE NUCLEAR REGULATORY COMMISSION'S (NRC)

Direct link to State Radiation Regulations and Legislation

Is Available at:

http://www.hsrd.ornl.gov/nrc/rulemaking.htm 


\section{LIST OF MISC. STATE PROGRAMS}

\section{CALIFORNIA}

Firearms Safety Device Testing and Laboratory Certification

Firearms Division

California Department of Justice

Public Inquiry Unit

P.O. Box 944255

Sacramento, CA 94244-2550

Phone: (916) 322-3360

Effective January 1, 2002, every firearm sold in California must be accompanied with a DOJ-approved firearms safety device. Sales to persons who demonstrate ownership of a suitable gun safe are exempt from this requirement. The Firearms Division is proposing regulations to implement handgun safety testing and laboratory certification requirements as provided in Penal Code sections 12087 through 12088.9.

\section{MINNESOTA}

Manure Testing Laboratory Certification Program

Minnesota Dept. of Agriculture

90 West Plato Blvd.

St. Paul, MN 55107

Phone: 651-297-7082 or 2200 or 1-800-967-2474 